\title{
Investigation of Various Factors Affecting Quantification and Characterization Accuracy of NOx Measurement at Near Zero Level in a CVS Sampling Set-Up
}

Samuel Abimbola Ogunfuye

West Virginia University, sao0008@mix.wvu.edu

Follow this and additional works at: https://researchrepository.wvu.edu/etd

Part of the Mechanical Engineering Commons

\section{Recommended Citation}

Ogunfuye, Samuel Abimbola, "Investigation of Various Factors Affecting Quantification and Characterization Accuracy of NOx Measurement at Near Zero Level in a CVS Sampling Set-Up" (2019). Graduate Theses, Dissertations, and Problem Reports. 3762.

https://researchrepository.wvu.edu/etd/3762

This Thesis is protected by copyright and/or related rights. It has been brought to you by the The Research Repository @ WVU with permission from the rights-holder(s). You are free to use this Thesis in any way that is permitted by the copyright and related rights legislation that applies to your use. For other uses you must obtain permission from the rights-holder(s) directly, unless additional rights are indicated by a Creative Commons license in the record and/ or on the work itself. This Thesis has been accepted for inclusion in WVU Graduate Theses, Dissertations, and Problem Reports collection by an authorized administrator of The Research Repository @ WVU. For more information, please contact researchrepository@mail.wvu.edu. 
2019

Investigation of Various Factors Affecting Quantification and Characterization Accuracy of NOx Measurement at Near Zero Level in a CVS Sampling Set-Up

Samuel Abimbola Ogunfuye

Follow this and additional works at: https://researchrepository.wvu.edu/etd Part of the Mechanical Engineering Commons 
Investigation of Various Factors Affecting Quantification and Characterization Accuracy of NOx Measurement at Near Zero Level in a CVS Sampling Set-Up

\title{
Samuel Ogunfuye
}

\author{
Thesis submitted \\ to the Benjamin M. Statler College of \\ Engineering and Mineral Resources \\ at West Virginia University
}
in partial fulfillment of the requirements for the degree of
Master of Science in
Mechanical Engineering

\author{
Marc Besch, Ph.D., Chair \\ Arvind Thiruvengadam, Ph.D. \\ V'yacheslav Akkerman, Ph.D. \\ Saroj Pradhan, Ph.D.
}

Department of Mechanical and Aerospace Engineering

West Virginia University

Morgantown, West Virginia

2019

Keywords: Near Zero, NOx Emissions Measurement, Quantification, Characterization, Accuracy, Low NOx Concentration Analyzer, Constant Volume Sampling (CVS).

Copyright 2019 Samuel Abimbola Ogunfuye 


\begin{abstract}
Investigation of Various Factors Affecting Quantification and Characterization Accuracy of NOx Measurement at Near Zero Level in a CVS Sampling Set-Up
\end{abstract}

\begin{abstract}
Samuel Ogunfuye
Accurate measurement of oxides of nitrogen (NOx) concentrations at near-zero levels using the constant volume sampling (CVS) system is a critical consideration in emissions quantification for engine and vehicle research and certification purposes. Various technological efforts and research are being implemented to ensure emissions from automobile and on-road transportation sources tend towards lower concentrations. This development has resulted in NOx concentration measurements to shift towards near-zero and thus, reach the detection limits of traditional measurement approaches and methods.

There are several factors that might deter achieving measurement accuracy at this level, as their effect becomes significant at low NOx concentrations. A number of selected factors were investigated as part of this study, including, making modifications to the current CVS measurement set-up to assess and ascertain the capability of the CVS in accurately quantifying NOx at near-zero concentration levels.
\end{abstract}

The effect of background variability of NOx and total hydrocarbons (THC) was investigated, with both species exhibiting variations in concentrations within a 10 minutes span across 3 days of measurements. Additionally, a dilution air filter was incorporated into the CVS dilution and engine intake air duct system to reduce and stabilize the NOx and THC concentrations which resulted in a 31\% average NOx across both analyzers and a 5\% average THC reduction. Furthermore, low NOx capable analyzers were also utilized to enhance the CVS measurement system. In general, an average drift error of $-1.7 \%$ was observed for both analyzers used over a 3-day period of measurements. Finally, the CVS dilution tunnel was heated to ensure a stable temperature across the tunnel within a range of $49^{\circ} \mathrm{C}$ to $59^{\circ} \mathrm{C}$ and thereby prevent any possible condensation inside the tunnel that could result in a reduced NOx and THC concentration measurement. Virtual NOx injections were carried out to simulate an average loss of NOx in the CVS sampling set-up. The analysis of the selected influencing factors showcased a tendency to affect the accurate characterization and quantification of NOx concentration measurements at near-zero levels. 


\section{Acknowledgements}

First and foremost, all praise and glory to God Almighty who has given me the grace and the enablement to start and finish this work. I will like to appreciate my parents $\mathrm{Mr}$ and $\mathrm{Mrs}$ Olugbenga Ogunfuye who has put so much efforts and resources in helping me attain this feat.

Being in this university I will like to appreciate my advisor Dr. Marc C. Besch for his unrelentless efforts towards the success of my master's journey I cannot ask for a better advisor ever. Appreciation to my committee member Dr. Arvind Thiruvengadam for his immense support and help regarding my academic journey, and not forgetting the knowledge acquired through you. My regards also to Dr. Akkerman for accepting to be part of my committee and for the relationship built thus far. I also want to thank Dr. Saroj for his enormous and unwavering assistance and contributions regarding this thesis work

My utmost regards to CAFEE director Daniel Carder whose help I cannot overlook in my voyage in becoming a graduate student in CAFEE. My immense appreciation goes to the wonderful family of CAFEE for their love and help all through the journey I will forever be grateful. My regards also to my colleagues Samuel Okeleye and Renata Moore nee Castiglioni for their help and assistance during the process of writing this thesis work. I really appreciate you both and am grateful. I will say to all friends and family have made in Morgantown WVU, including RCCG Living-Spring, and within the US in my short time here and even overseas, am happy and forever grateful that our paths crossed.

I want to specially appreciate The Ogunfuye's, The Olaoye's, The Adewunmi's, The Oshiga's, The Tijani's, AutoSparemall, CAC-PHP and to all my friends and family in Nigeria and diaspora time will fail me to mention you all. I cannot thank you enough for having you all, as you were all there when the going got tough. All your support and help will never be forgotten.

I can never forget to mention my EDUCATION USA family Ms Adebayo, Ms Atobatele, Ms Suwa, Ms Malete, Ms Uwadileke and Ms Adejumobi and all EDUUSA members. I really appreciate you for the efforts and love shown and for also being part of my success story. You all contributed in making me see the light at the end of the tunnel when all seems blurry (and all I could only see is thick darkness). You made me believed and ensured my dream of studying in the U.S come true. 


\section{Table of Contents}

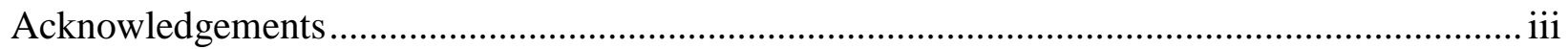

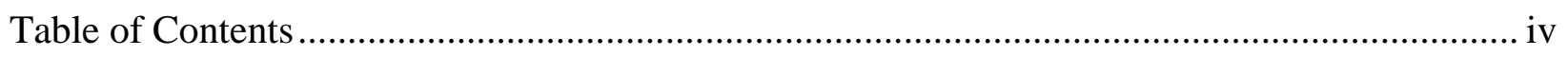

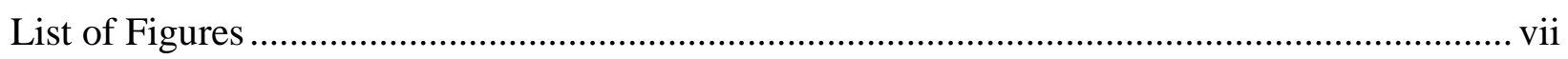

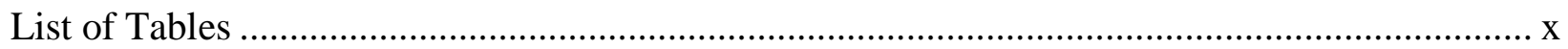

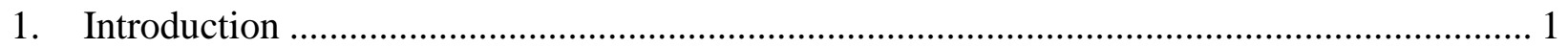

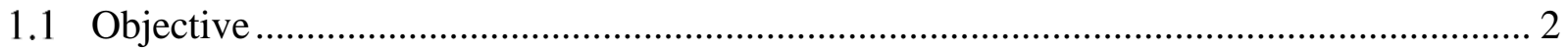

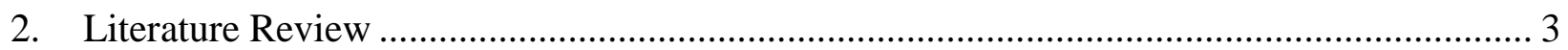

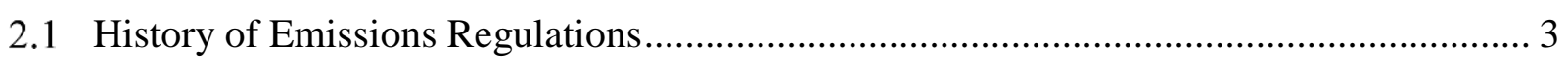

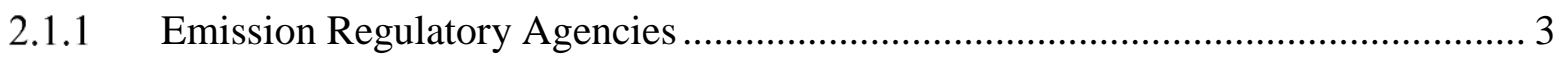

2.1.2 Automobile Emission Standards and Regulations ................................................. 3

2.1.3 Overview of NOx Emission Regulations and Standards ......................................... 4

2.1.4 Emission Standards for Heavy Duty Vehicles........................................................ 6

2.2 Technologies for NOx Emission Reduction and Control …………................................. 7

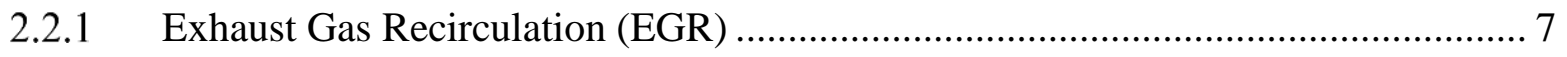

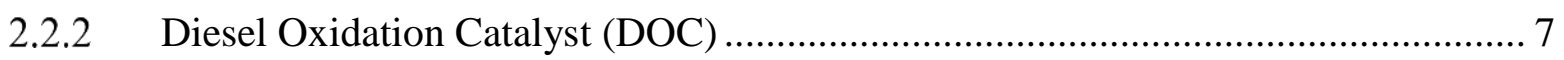

2.2.3 Three Way Catalyst (TWC) Converter ............................................................... 7

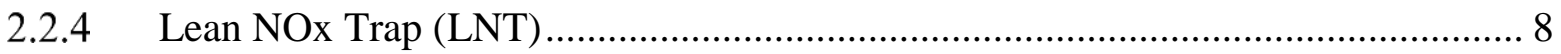

2.2.5 Selective Catalytic Reduction (SCR) ............................................................... 8

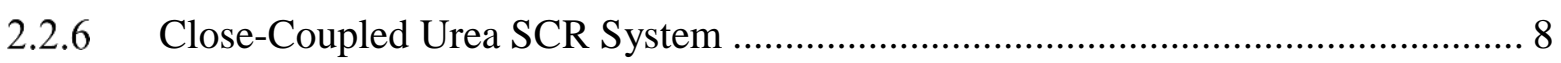

2.2.7 Other NOx Reduction Technologies and Strategies ............................................ 9

2.3 Automotive Exhaust Emission Measurement ……………………................................ 9

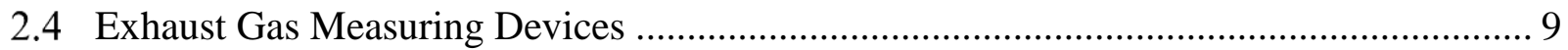

2.4.1 Gas Analyzers Working Principles ................................................................. 10

2.4.2 Chemiluminescence Detector Principle .............................................................. 10

2.4.3 Non-Dispersive Infra-Red (NDIR) Detectors Principle ………………................ 11

2.4.4 Fast Flame Ionization Detector Principle ………….......................................... 12

2.5 Exhaust Gas Measuring Devices Terms …………………........................................ 13

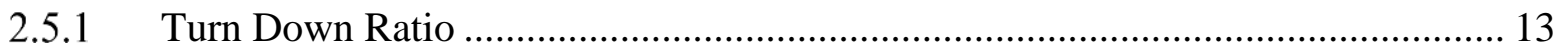

2.5.2 Limit of Detection and Quantification ........................................................... 14

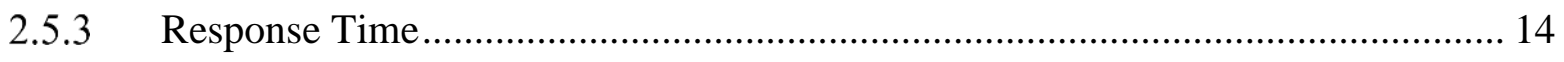

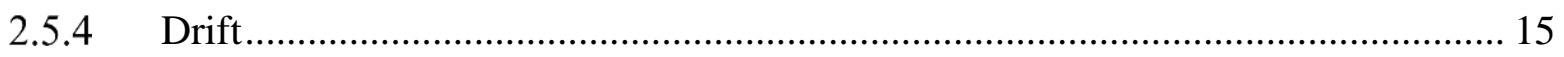

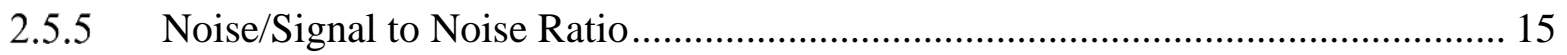




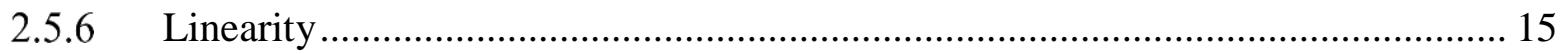

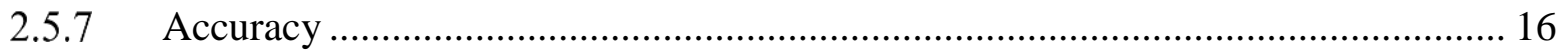

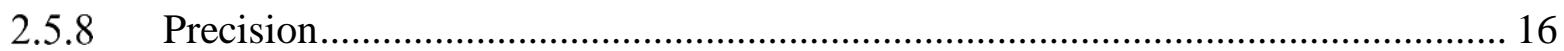

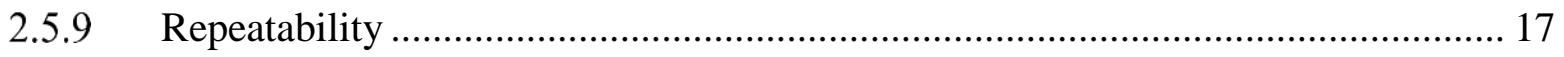

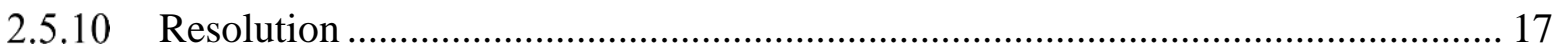

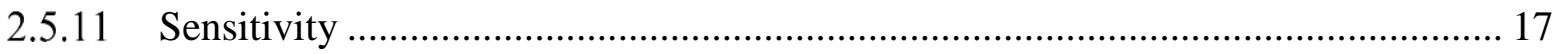

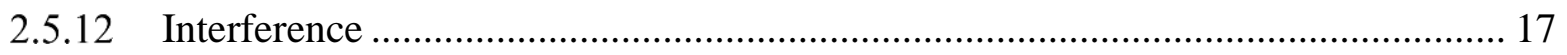

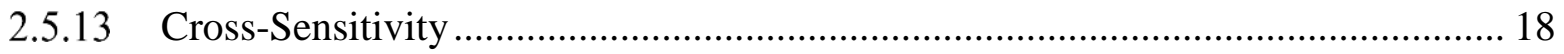

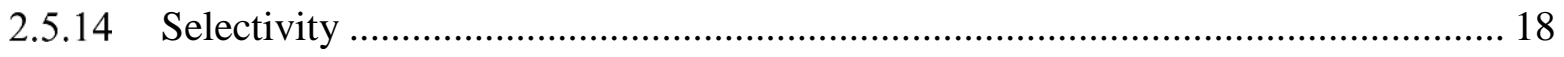

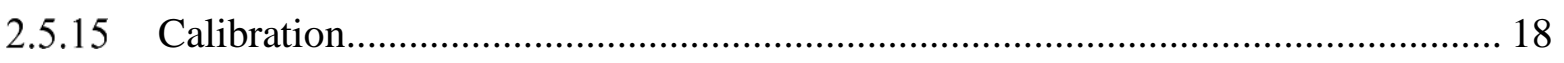

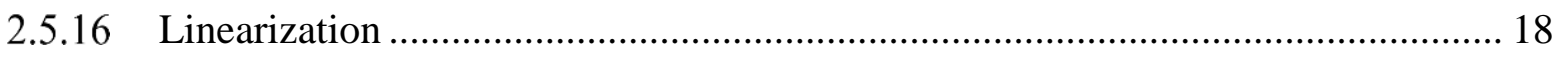

2.6 Emission Measurement and Sampling System ……………………………………...... 18

2.6.1 Raw/Direct Exhaust Sampling .............................................................................. 19

2.6.2 Dilute Exhaust Sampling System ……………............................................. 19

2.7 Constant Volume Sampling Measurement System........................................................ 20

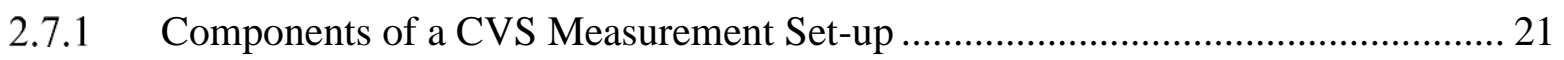

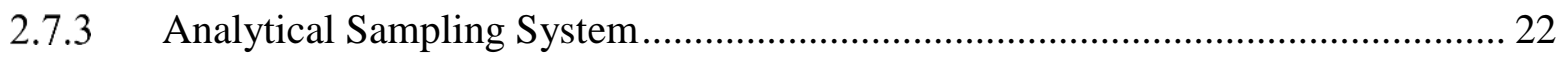

2.7.4 Operating Systems and Data Acquisition (D.A.Q) .............................................. 22

2.7.5 CVS System Calibration and Verification ......................................................... 22

2.8 Challenges of the CVS for Emissions Measurement ....................................................... 24

2.9 Limitation of the CVS System in NOx Measurement .................................................. 24

2.10 Challenges with NOx measurement at Near Zero Level.............................................. 25

2.11 Enhancement of the CVS system Set-Up for Accurate Low NOx Measurement.......... 27

3. Experimental Set-up and Procedures............................................................................. 28

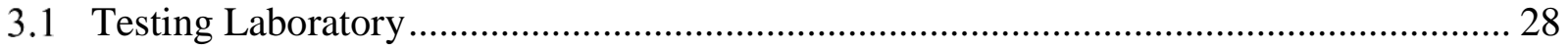

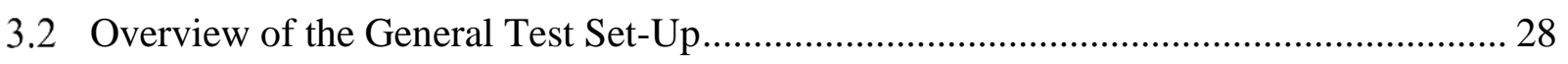

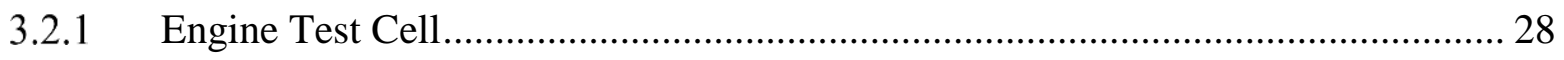

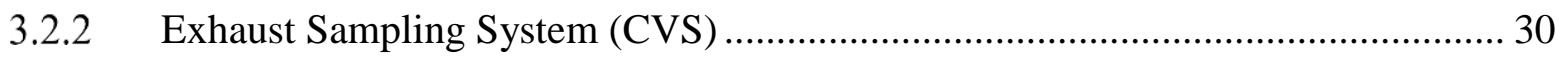

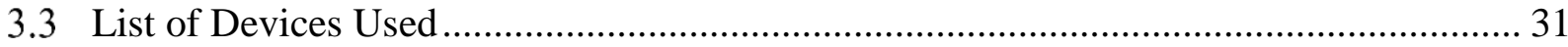

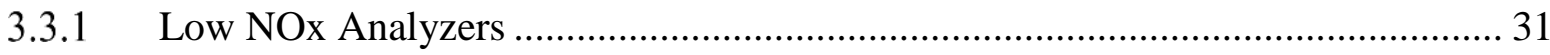

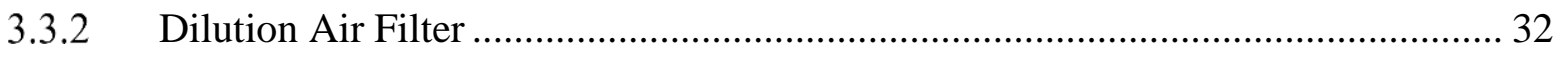

3.3.3 Proportional Bag Sampling System ……………….......................................... 33 


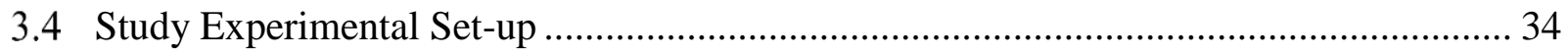

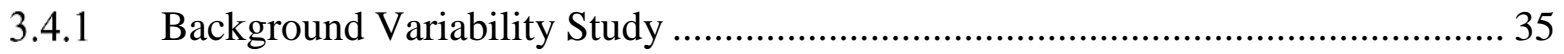

3.4.1.1 Dilution Air Filtration System ………………….................................. 36

3.4.1.2 Proportional Bag Sampling …………………….................................... 37

3.4.2 Low Concentration NOx Analyzer Study …………………………................... 38

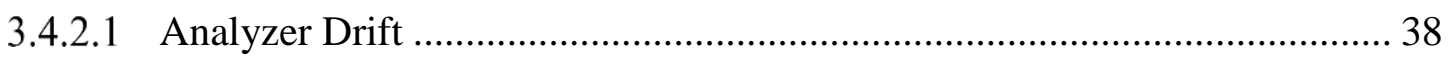

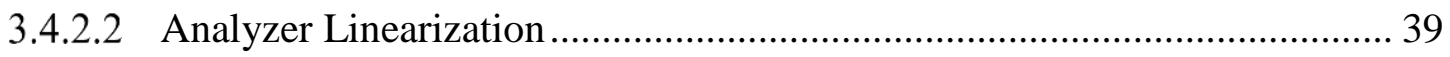

3.4.3 Virtual NO/NO2 Injection Checks..................................................................... 39

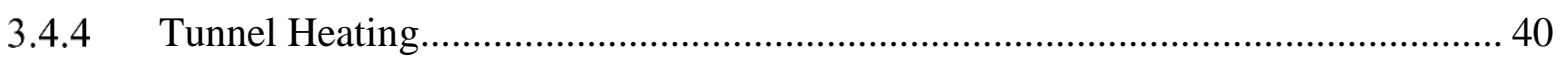

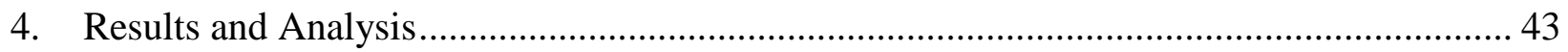

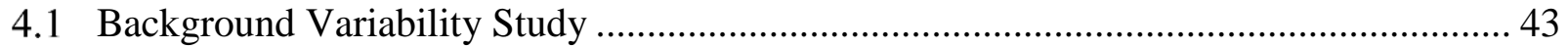

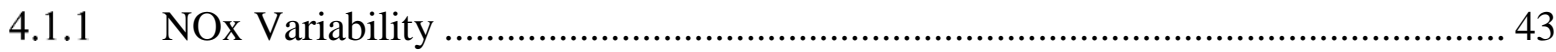

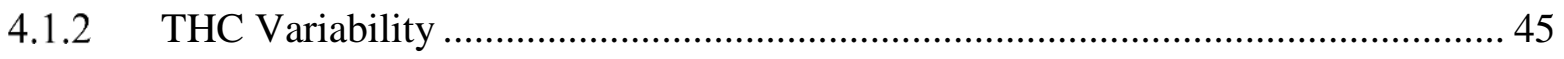

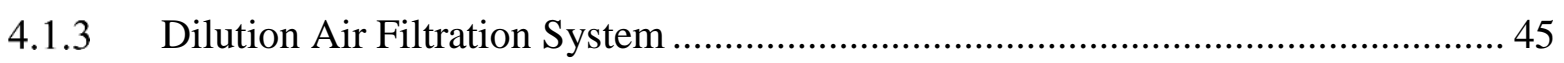

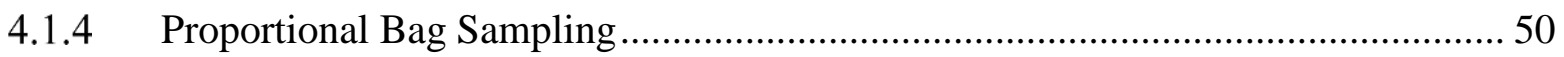

4.2 Low Concentration NOx Analyzers Comparison ......................................................... 52

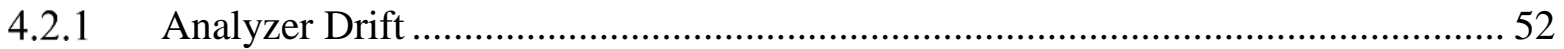

4.2.2 Linearization Accuracy Comparison ……………............................................. 55

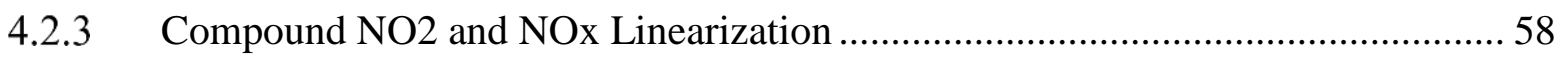

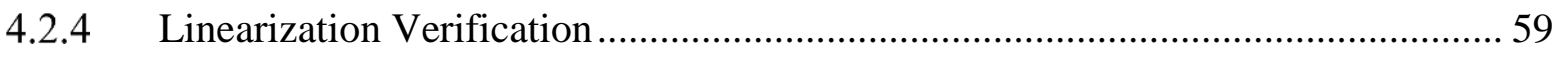

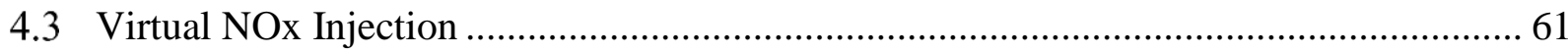

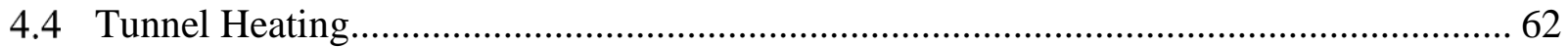

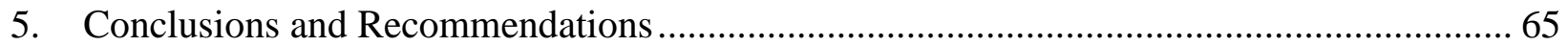

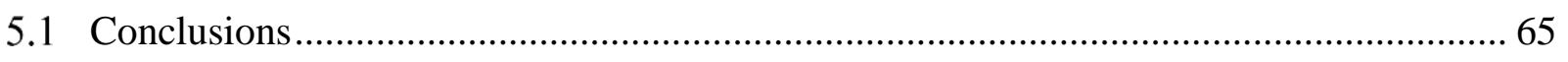

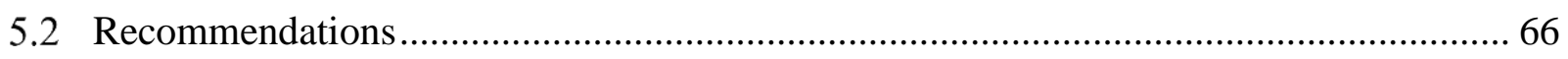

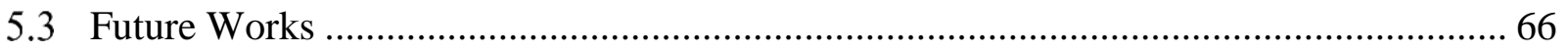

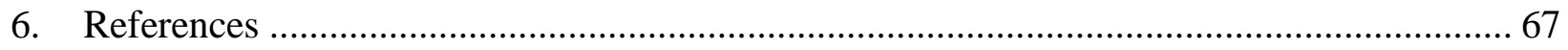

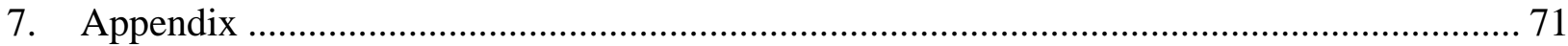




\section{List of Figures}

Figure 1 - Trend of U.S. On-road Mobile NOx Emission Sources …..................................... 4

Figure 2 - NOx Emission Comparison for Trucks used for Port Operations …….................. 6

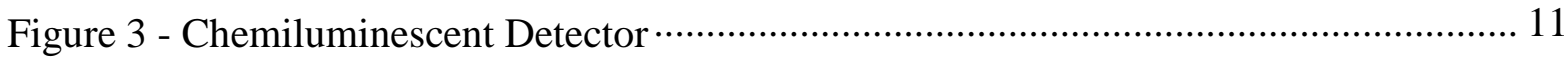

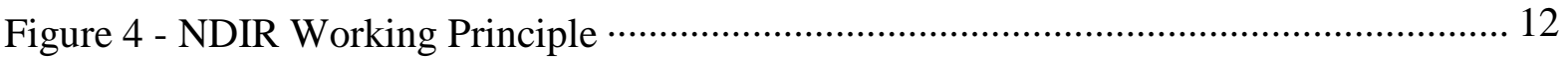

Figure 5 - Fast FID Measurement Principle …….......................................................... 13

Figure 6 - LOD and LOQ Comparison of a Measuring Device …..................................... 14

Figure 7 - Relationship Between Different Expression of Accuracy ….............................. 16

Figure 8- Overview of the CVS Measurement System Set-up ......................................... 20

Figure 9 - Effect of Water Vapor on Relative Quenching of NO ….................................. 26

Figure 10 - Effect of $\mathrm{CO}_{2}$ Concentration on Measured NO Value …................................ 26

Figure 11 -Engine Test Cell: Dyno [1], EATS [2],2015 DD15 engine [3], Connection to CVS

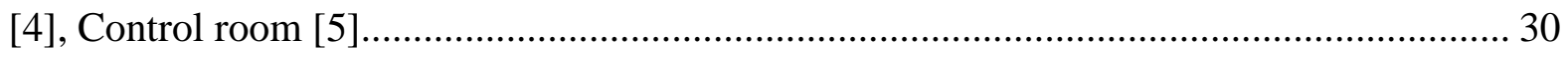

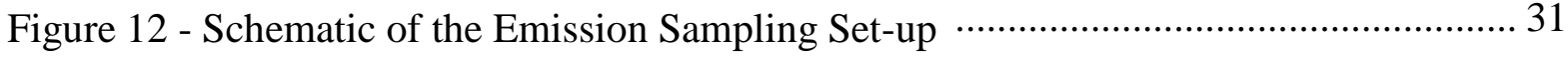

Figure 13- Trace level NOx analyzers: Brandgaus 7705[1], Ecophysics CLD64 [2], MKS FTIR

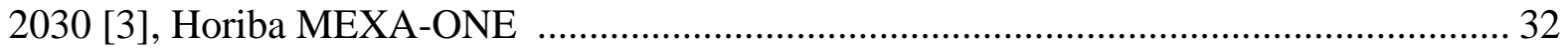

Figure 14 - Schematic of the Dilution Air Filter …......................................................... 33

Figure 15 - Proportional Bag Sampling System: Mass flow controller [1], Vacuum pumps [2],

Robust control of filling [3], Bagging System [4]. …..................................................... 34

Figure 16- CVS Modifications Set-up: Dilution air filter [1], Proportional bag sampling [2],

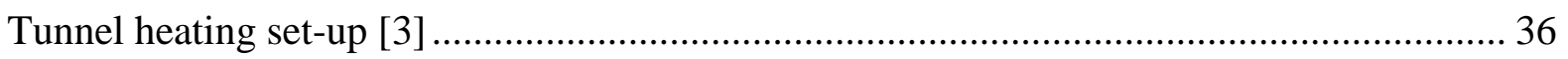

Figure 17 - Dilution air Filtration Pre and Post Sampling .............................................. 37

Figure 18 - Low concentration NOx analyzer set-up: DAQ system [1], Ecophysics CLD64 [2],

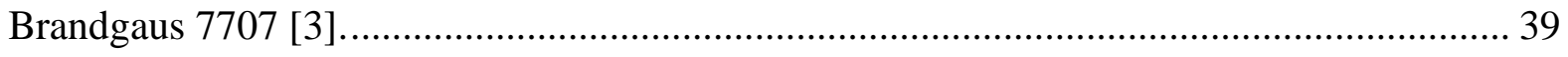

Figure 19- Tunnel Heating Set-up: Thermocouples locations [1,2,3], Dilution air and exhaust mixing chamber entrance [1], Hot water inlet [4], Water Outlet [5], Sampling Plane [6], Tunnel

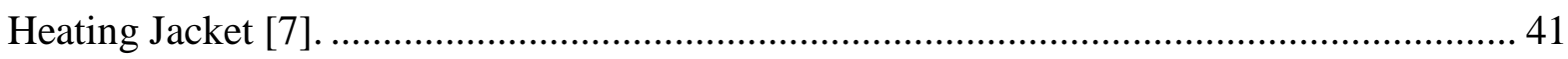

Figure 20- Schematic of the Tunnel Heating Set-up ................................................... 42

Figure 21- Variation of Background NOx Concentration across Day 1 .............................. 43 
Figure 22- Variation of Background NOx Concentration across Day 2(Test 2) .................. 44

Figure 23-Background THC Concentration: Day 1 and Day 2 ...................................... 45

Figure 24- Dilution Air Filter \% Reduction in ambient NOx : Test 1 [1], Test 2 [2],Test 3 [3],

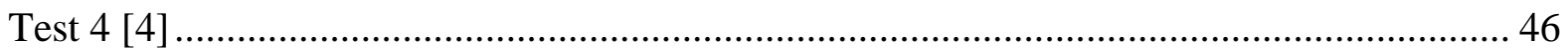

Figure 25- Effect of Increase in Temperature on \% NOx Reduction ................................ 47

Figure 26- Effect of Increase in Relative Humidity on \% NOx reduction .......................... 48

Figure 27 - Dilution Air Filter \% Reduction in ambient THC: Test 1 [1], Test 2 [2], Test 3 [3],

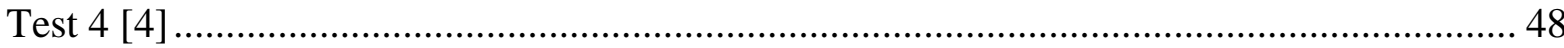

Figure 28 - Effect of increasing Temperature and Relative Humidity on \%THC Reduction. 49

Figure 29 - Percent Error Difference in NOx Measured to CVS Measurement .................... 51

Figure 30 - ANOVA and Student-T Comparison for CVS and FTIR NOx Measurement at

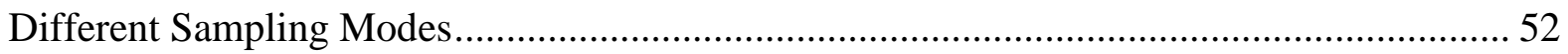

Figure 31- Ecophysics NOx Analyzer Daily Hourly Zero Drift........................................ 53

Figure 32- Brandgaus NOx Analyzer Daily Hourly Zero Drift......................................... 53

Figure 33- Ecophysics NOx Analyzer Daily Hourly Span Drift ....................................... 54

Figure 34- Brandgauss NOx Analyzer Daily Hourly Span Drift...................................... 54

Figure 35- NOx Analyzers Linearization using NOx .............................56

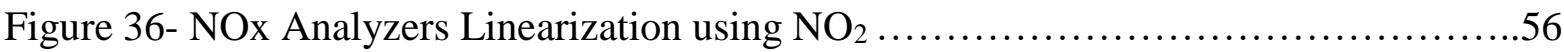

Figure 37- Percent NOx Measured by Analyzer using NOx ..........................57

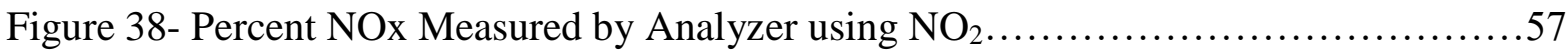

Figure 39- Analyzer Compound NO2 and NOx linearization accuracy comparison..........58

Figure 40- Analyzer Compound NO2 and NOx linearization Percent Measured...........59

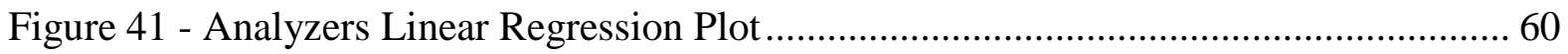

Figure 42-Comparison of Injected Mass of NOx to Recovered Mass of NOx..............61

Figure 43- Percent Error in Recovered Mass of NOx..............................62

Figure 44 - Optimization of Tunnel Heating to minimize temperature difference.........63

Figure 45 - Effect of heating on Relative Humidity and Temperature at Sample Plane ....... 64

Figure A1 - Day 2 Test 1 Background NOx Variation (Brandgauss) ....................71

Figure A2 - Day 2 Test 1 Background NOx Variation (EcoPhysics) .....................71

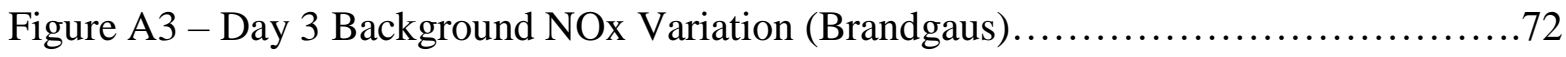

Figure A4 - Day 3 Background NOx Variation (EcoPhysics)..........................72 
Figure A5 - JMP Output of ANOVA and Student-T test comparison of CVS measurements at four Different Bag Sampling modes............................................. 74

Figure A6- JMP Output ANOVA and Student-T test comparison of FTIR measurements at four Different Bag Sampling modes............................................75

Figure A7 - JMP Output ANOVA and Student-T test comparison of Brandgaus measurements at four Different Bag Sampling modes....................................76

Figure A8- JMP Output ANOVA and Student-T test comparison of Ecophysics measurements at four Different Bag Sampling modes. .77

Figure A9 - Summary of Brandgauss Analyzer Linear Regression Model.................78

Figure A10 - Summary of Ecophysics Analyzer Linear Regression model................78 
List of Tables

Table 1 - USEPA \& CARB NOx Emission Standard for Heavy Duty Diesel Engines .......... 5

Table 2 - Gas Species and Measurement Working Principle ….................................... 1010

Table 3 - Calibration Data and Measurement Tolerance for CVS Set-up …...................... 23

Table 4 - Error Variation with Decreasing Concentration …............................................ 25

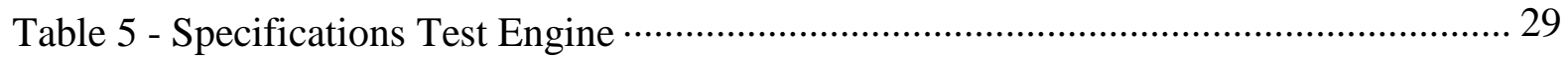

Table 6- Summary of Experiments Carried Out .................................................................. 35

Table 7 -Dilution Air Filter Average NOx and THC Reduction with Different Testing

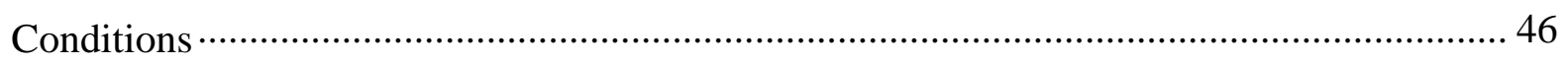

Table 8- Summary of Average NOx Reduction Across Testing Conditions ......................... 47

Table 9- Summary of Average THC Reduction Across Testing Conditions........................ 49

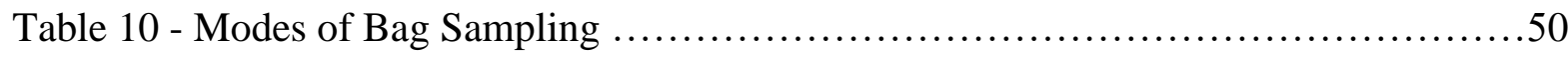

Table 11- Summary of Analyzers Measurements Least Square Regression ....................... 60

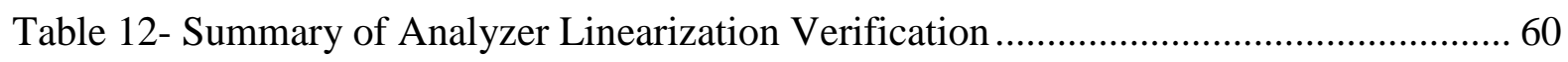

Table 13 - Water Temperature across the Heated Tunnel …............................................ 63

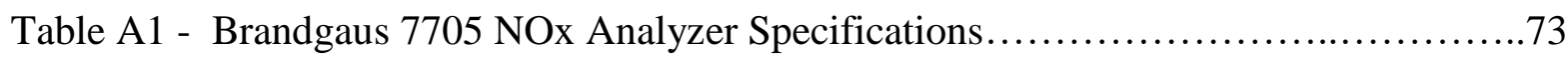

Table A2 - Ecophysics CLD 64 NOx Analyzer Specifications.............................73 


\section{Introduction}

Stringent automotive exhaust emissions regulations have been set because of high health risks caused by such emissions, and according to the National Ambient Air Quality Standards (NAAQS) these air pollutions must be regulated for cleaner atmosphere and healthy environment. Another major challenge is adverse weather and climate effects such as smog which is created in the presence of ozone in the atmosphere when oxides of nitrogen $(\mathrm{NOx})$ and oxygen $\left(\mathrm{O}_{2}\right)$ reacts in the presence of sunlight. This has posed a problem to the air quality and visibility in major cities such as Beijing, Shanghai, Los Angeles and New Delhi. The recent development about strict emission policies have resulted in engine manufacturers implementing changes in engine designs and aftertreatment systems which has invariably lowered emissions from automobiles.

The need to develop means to accurately measure emissions at near zero level is the focus of most emissions research. As newer vehicles overtime had to be certified on a stricter basis compared to earlier vehicles, to ensure they meet these emissions limits prior to them being approved for use. These policies were efforts put in place to curb vehicles emission which was termed a major pollutant source causing adverse weather conditions and other health related challenges. These certification process must be according to the United States Environmental Protection Agency (USEPA) emission regulations for vehicles in the United States (US). This excludes California which uses California Air Resource Board (CARB) standards as guidelines to certify vehicles plying in and out of the state of California which are usually more stringent compared to the USEPA standards. The current NOx emission standards for automobiles in the U.S is the 2010 standard which stands at $0.2 \mathrm{~g} / \mathrm{bhp}-\mathrm{hr}$ and the projected standard is the optional 2015 NOx emission standards which is set at 0.02g/bhp-hr. Enormous technological efforts and strategies has been carried out by manufacturers, researchers, and government agencies to reduce NOx emission from automobile exhaust. Overtime the emission measurement procedures has been established and reviewed, but the devices and means to measure these emissions has not really changed much to meet up with this near zero emissions limit, the reason for this has been that measurement at this near zero level have its inherent challenges. Earlier works has shown that measurement difficulties attributed to NOx becomes significant when the NOx emission concentration range goes low $(\leq 100 \mathrm{ppm})$ [1].The precision, accuracy and repeatability of measurements gotten becomes questionable when the NOx emission concentration range tends towards near Zero ( $\leq 30 \mathrm{ppm})$. 
Measurement of NOx with high level of exactness at this low level is necessary as efforts are being made to ensure vehicle NOx emission production tends to near zero. These efforts put in place have created a need for robust measures to ensure accuracy and precision in measuring this low NOx emissions, as the future of automobile emission certifications relies typically on the ability to measure these near zero emissions accurately using standard measurement set-up. The Constant Volume Sampling System (CVS) is the current standard measurement device used for automobile emission certification and quantifying engine exhaust emission [2]. The principle of operation which involves dilution of the exhaust concentration to avoid condensation or loss of some emission species, worked quite well in the quantification and characterization of emissions with high level of NOx emission concentration range [3]. At near zero level it is not certain the CVS set-up has the utmost capabilities in absolutely measuring exhaust gas precisely at this low NOx concentration range accurately due to its mode of operation. Factors and conditions contributing to errors in NOx emission quantification and characterization are therefore needed to be investigated.

\subsection{Objective}

The objective of this work is centered on accurate measurement of automobile emissions at low levels using the standardized measurement procedures. Traditional measurement approaches are reaching their detection limits and as such accuracy, precision and repeatability in measuring and characterizing emissions at this level might be questionable. The specific objective of this research will be investigating selected factors affecting quantification and characterization of NOx measurement near zero level in a CVS sampling set-up.

The focus of this study will be towards investigating the effect of the following selected factors on the accuracy of NOx measurement:

i. Background Variability Study

a. Dilution Air Filtration

b. Proportional Bag Sampling

ii. Low Concentration NOx Analyzer

iii. Virtual NO/NO2 Injection

iv. Tunnel Heating 


\section{Literature Review}

As this study investigates the capabilities of current measuring system, it also includes possible modifications that might be needful within the Constant Volume Sampling (CVS) measurement setup to attain precision, accuracy and repeatability in quantifying and characterizing emissions at near zero level. An overview of earlier works in this area is needful for guidance.

\subsection{History of Emissions Regulations}

The current stringent USEPA emissions regulations has resulted to a drastic decrease in the emissions limit from automobile vehicle exhaust and advancement in after-treatment technologies. This standard has developed overtime from the mid- $20^{\text {th }}$ century when regulations were first implemented to reduce the health hazards associated with polluted air and mitigate the effects of these pollutants on poor weather visibility. The major source of this pollution was affiliated to emissions from automobile exhaust. Hence in 1961, the first automobile emission control technology was developed and by 1966 the first automobile emission regulations were put in place in the United State of America (US) [4].

\subsubsection{Emission Regulatory Agencies}

The U.S government at state and federal levels established various regulatory agencies to control the pollution from automobile exhaust and to be able to meet up with the NAAQS. By 1967 the CARB was established while the USEPA was established in 1970 amongst other regulatory agencies to ensure the implementation and compliance of these emissions standards [4]. While the USEPA and CARB are responsible for the regulations of the air emission pollutants from automobile exhaust. The U.S Department of Transportation (D.O.T) is responsible for the regulation of average automobile's fuel economy which they set for each manufacturer based on Corporate Average Fuel Economy Standard (CAFE) as emissions control must be done with the least fuel consumption possible. Other international regulatory agencies include the European Union Emission Standards, Air Pollution Dispute Resolution Act, and Environment Canada of Canada, which also follow similar standards.

\subsubsection{Automobile Emission Standards and Regulations}

Emission standards are promulgated enforcements which are set to control and limit the amount of emissions from different emission sources which can include vehicles, power plants, 
and manufacturing industries [5]. The automobile emission standards apply to automobiles sources to limit the amount of pollutants from the exhaust gas of a vehicle, these are usually enacted for a cleaner air and healthier environment. Automobile emissions can be categorized mainly into regulated and unregulated emissions according to the EPA and CARB standards. The regulated automobile emissions standards according to the EPA and CARB are $\mathrm{NO}_{\mathrm{X}}$, Particulate Matter (PM), Total Hydrocarbon (THC), Carbon-monoxide (CO) and Greenhouse gas (GHG) emission Carbon-dioxide $\left(\mathrm{CO}_{2}\right), \mathrm{CH}_{4}$ and $\mathrm{N}_{2} \mathrm{O}$ [6]. One of the challenges of the current emission reduction and control technologies has been the effect of increasing fuel consumption while trying to decrease and meet emission standards.

\subsubsection{Overview of NOx Emission Regulations and Standards}

NOx emission standards and regulations has evolved over the years which has contributed in achieving a cleaner and healthier environment with less air pollutant in the U.S. This EPA standards has led to significant decrease in the amount of yearly NOx emissions from on-road mobile air pollution sources. This currently serves as one of the major pathways for states to achieve the set NAAQS standards.

U.S National Nitrogen Oxides Emissions: Mobile Source-On-

Road

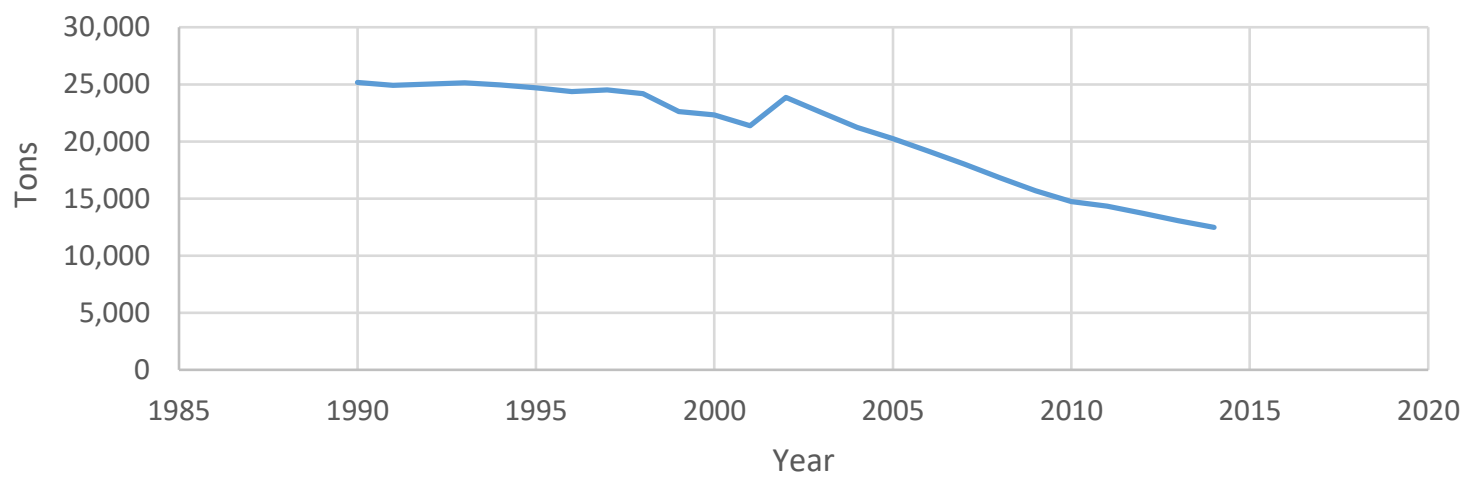

Figure 1 - Trend of U.S. On-road Mobile NOx Emission Sources [7].

NOx standards in U.S has over the years reduced tremendously with one of the notable stringent standards set in 2010 , with a certification value of $0.2 \mathrm{~g} / \mathrm{bhp}-\mathrm{hr}$. This occurrence led to development and utilizing of new after-treatment technologies including the Selective Catalytic Converter (SCR), amongst other innovative strategies relating to efficient combustion and better 
engine design. While also introducing novel technologies to ensure compliance with these automobile NOx emission standards. As the standards tends towards near zero, the technology in achieving them requires robustness and means to measure and characterize these emissions has to be accurate and precise. Amidst all these efforts and process to attain the 2010 NOx emission standards the CARB established the severe optional low-NOx standards in 2013 [8]. This standard is a precursor of the USEPA future NOx emission standards as the USEPA do adopt these standards later after the CARB might have implemented it and make it mandatory.

Table 1 - USEPA \& CARB NOx Emission Standard for Heavy Duty Diesel Engines [9]

\begin{tabular}{|c|c|}
\hline Model Year & NOx (g/bhp-hr) \\
\hline $1985-1989$ & 10.7 \\
\hline 1990 & 6.0 \\
\hline $1991-1997$ & 5.0 \\
\hline 1998 & 4.0 \\
\hline 2004 & $2.5(\mathrm{NOx}+\mathrm{NMHC})$ \\
\hline 2007 (Phasing till 2010) & 0.20 \\
\hline 2015 (Optional) & 0.02 \\
\hline
\end{tabular}

There has been drastic reduction in the USEPA emission standards over the years with NOx in particular, as regards the current NOx automobile exhaust emission standards for heavy duty vehicles. This decrease invariably assumes that the concentration of the NOx emissions from automobile exhaust has reduced to a considerable low range over the years. As there is higher tendency of attaining near zero limits when the optional CARB low NOx $0.02 \mathrm{~g} / \mathrm{bhp}$-hr certification standard becomes mandatory for CARB and for the US emissions regulations in 2024 [9]. Recent technological efforts shows that the near zero limits can be attained as some heavy duty natural gas engines has been certified based on the optional low NOx standards [10].In couple of years it can be inferred that the automobile exhaust NOx emission concentration will range below 10ppm, and at most operating conditions excluding the cold-start, low load, low temperature operating regions the value of emitted NOx will be near zero. Current research efforts are towards ensuring emission spikes observed at certain operating conditions such as low load, low temperature and cold start attains the near zero limits as most of the NOx emission spikes are noticed during these operating conditions. Having a robust solution at these conditions will reduce emissions to a great 
measure and as such the near zero NOx limit will be a basic trend at all operating conditions of the vehicle. This envisaged development therefore requires a robust process, to accurately measure this trend towards a new "near zero NOx emission era" for certification, quantification and characterization purpose.

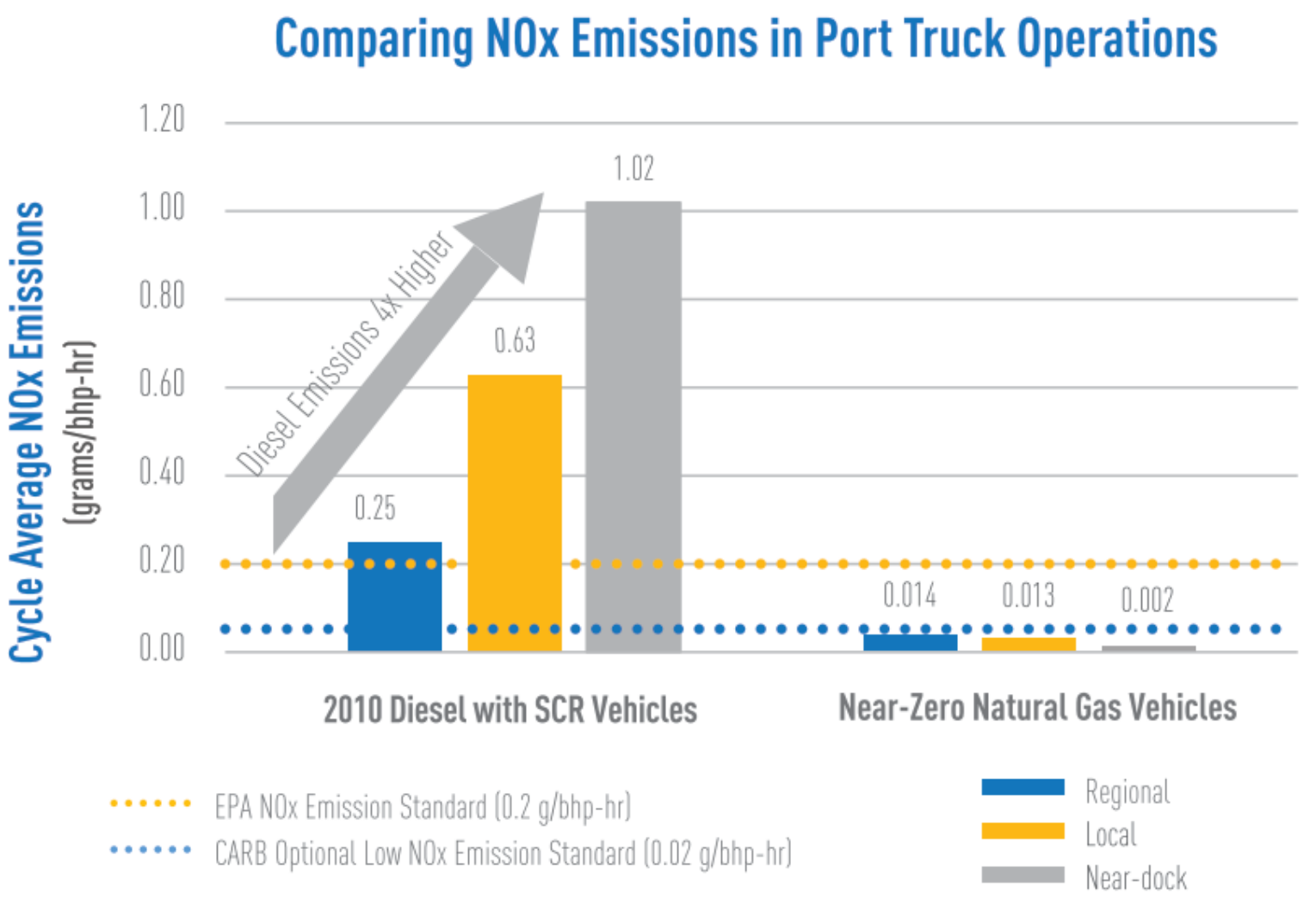

Figure 2 - NOx Emission Comparison for Trucks used for Port Operations [11]

The NOx emission standard values showcased in Table 1 above are evident that the current emission standards will make newer vehicles exhaust emissions concentration range tends towards the near Zero range $<50 \mathrm{ppm}$ [1]. To be able to achieve the $0.2 \mathrm{~g} / \mathrm{bhp}$-hr current standards and achieve the optional 0.02g/bhp-hr automobile NOx exhaust emission standard in the U.S.

\subsubsection{Emission Standards for Heavy Duty Vehicles}

NOx emission standards for on road diesel engines for heavy duty vehicles has tightened gradually over the years with over $99.8 \%$ reduction from 1985 as depicted in Table 1 using various technological approach and strategies. Heavy duty engines due to the nature of their combustion and operating conditions makes them more susceptible to emit higher NOx to the atmosphere. The 
current SCR technology on a stand-alone basis is not self-sufficient to reduce emission to meet up with the current NOx emission standard. To ensure compliance with future NOx emission standards which are tending towards low NOx regions [12] one of the proposed Exhaust Aftertreatment System (EATS) technologies for heavy duty vehicles to attain this low NOx standards is the closed couple SCR technology [13]. Other efforts includes making improvements to the current SCR after-treatment system which might include a robust operating temperature of the SCR catalysts to ensure wider optimum temperature range, to ensure an improved SCR aftertreatment system [14].

\subsection{Technologies for NOx Emission Reduction and Control}

There have been developments regarding emission control technologies to reduce emission and meet up with the stringent emission standards which had evolved overtime. Some of the emission control devices installed in automobiles overtime includes the Positive Crankcase Ventilation System (PCV) system in 1961 [15]. Some of the notable development was the elimination of leaded fuel for automobile use which made it possible to install a Catalytic Converter for automobile emission control, others include Lean NOx Trap [LNT], Exhaust Gas

Recirculation (EGR), Diesel Oxidation Catalyst (DOC), Three Way catalyst (TWC), SCR and Diesel Particulate Filter (DPF).

\subsubsection{Exhaust Gas Recirculation (EGR)}

An emission control strategy which is utilized to reduce the amount of NOx produced in automobiles as NOx production is favored by high combustion temperature. Some part of the exhaust is returned into the engine combustion chamber as an intake air, this reduces the combustion temperature of the exhaust and reduces the amount of NOx emitted.

\subsubsection{Diesel Oxidation Catalyst (DOC)}

A device used for emission control as it oxidizes the automobile exhaust and ensures that the product is non-harmful. It oxidizes the $\mathrm{CO}$ and Hydrocarbons (HC) and tends to oxidize the Nitric Oxide (NO) to $\mathrm{NO}_{2}$, while also increasing the amount of $\mathrm{NO}_{2}$ in the exhaust for subsequent use by other emission control device downstream of exhaust.

\subsubsection{Three Way Catalyst (TWC) Converter}

An emission control device suited for rich burn engines as the converter requires stoichiometric or slightly rich combustion to operates effectively. It concurrently reduces the $\mathrm{NO}_{\mathrm{X}}, \mathrm{CO}$ and THC 
in an exhaust by converting the NOx into nitrogen $\left(\mathrm{N}_{2}\right)$ and $\mathrm{O}_{2}$ using a rhodium catalyst. The excess $\mathrm{O}_{2}$ is reacted with $\mathrm{CO}$ to produce $\mathrm{CO}_{2}$, while the THC particles and other unburned fuel are burned off by the high temperature of the exhaust gas.

\subsubsection{Lean NOx Trap (LNT)}

This is an after-treatment device used for NOx emissions control by trapping the NOx emissions from automobile exhaust through a reduction reaction on a catalyst. The NOx is typically stored on the catalyst usually Barium Nitrate $\left(\mathrm{Ba}\left(\mathrm{NO}_{3}\right)_{2}\right)$ during lean conditions. The NOx is released based on different adsorbing strategy which can be either active or passive, and occurs during fuel rich condition as a form of regeneration [16].In some applications the LNT are used concurrently with the SCR system for NOx emission control at low load, low temperatures and during cold start period. As the LNT works best at low temperature compared to high temperature [17]. The LNT traps the NOx at this low temperature condition and releases it when the temperature is higher for the SCR to function optimally.

\subsubsection{Selective Catalytic Reduction (SCR)}

SCR were prior used in power plants for emission control, but this technology has been adapted for automobile use. It is a major component of the after-treatment system designed for NOx emission reduction and control. This device has approximately $90 \%$ NOx conversion efficiency which is usually achieved by dosing urea into the exhaust gas system. This urea converts the NOx into $\mathrm{N}_{2}$ and $\mathrm{H}_{2} \mathrm{O}$. The SCR requires an optimum working temperature as the catalyst can only work at some designated temperature. This is due to thermal stresses and possibility of water vapor present in the exhaust gas which are conditions associated with operating at lower temperature and can result in the damage of the catalyst.

\subsubsection{Close-Coupled Urea SCR System}

This is an engine after-treatment technology in which the SCR catalyst is coated on the DPF. it is otherwise called SCR/F or SDPF (SCR coated on DPF) after-treatment technology. The SCR/F system is attached close to the engine to ensure the SCR catalyst is exposed to hot exhaust gas. This is needful for rapid attainment of temperature for optimal operation, and as such increase fuel efficiency as less fuel is used for warming up due to its proximity to the engine which ensures higher temperature [18]. 


\subsubsection{Other NOx Reduction Technologies and Strategies}

Asides the above after-treatment technologies some other robust strategies utilized includes: improved thermal management of exhaust gas for efficient energy utilization, fuel-based warm-up strategies: combination of main injection and multiple after-injections, ameliorated engine management practices: elevated idle speed, rapid catalyst light off and better urea dosing strategies [19]. Some of the technologies adopted includes enhanced after-treatment technology choices and configurations: enhanced and efficient catalyst [20].

\subsection{Automotive Exhaust Emission Measurement}

These are various emissions measurement systems designed for automobile exhaust which are used in the quantification and characterization of emissions from these sources. The measurement is mainly carried out for verification and certification purpose and as such require high level of accuracy in measuring the emission species from these air pollution source [21]. These can be grouped majorly into two types based on purpose: Laboratory Testing and Field Testing [22]. The focus of this work is more particular on the laboratory testing which are used for certification and compliance testing as they are highly standardized for emissions measurement. These testing are usually carried out in an emission test cell which is well monitored and controlled for accurate emission quantification.

\subsection{Exhaust Gas Measuring Devices}

Exhaust gas measuring devices entails various instruments and analyzers used in quantifying various regulated emission specie with their specific measurement principles. These devices usually are supplied a sample of the total exhaust gas which would have been well mixed thereby making the measurement representative of the concentration of the exhaust gas measured. Gas analyzers have capabilities, limitations, response time, accuracy and measurement operations and specie they are best suited for as summarized in

Table 2 . 
Table 2 - Gas Species and Measurement Working Principle [22]

\begin{tabular}{|c|c|}
\hline Gas Specie & Gas Analyzer Principle \\
\hline NOx & $\begin{array}{c}\text { Chemiluminescence detector (CLD), Non- } \\
\text { dispersive ultra-violet (NDUV), Fourier transform } \\
\text { infra-red (FTIR) }\end{array}$ \\
\hline Total HC & Flame ionization detector (FID), FTIR \\
\hline $\mathrm{CO}$ & Non-dispersive infra-red (NDIR), FTIR \\
\hline $\mathrm{CO}_{2}$ & Non-dispersive infra-red (NDIR), FTIR \\
\hline $\mathrm{N}_{2} \mathrm{O}$ & Non-dispersive infra-red (NDIR), FTIR \\
\hline
\end{tabular}

\subsubsection{Gas Analyzers Working Principles}

Measuring techniques utilized in gas analyzers which are 1065 compliant measuring instruments includes CLD, NDIR, NDUV and FID. These are also utilized in continuous emission monitoring system (CEMS) of exhaust sample emission constituents.

\subsubsection{Chemiluminescence Detector Principle}

This is a measurement principle used for NOx measurement, the chemiluminescence principle basically measures the concentration of NO and not the total NOx. The NOx is converted to NO before it reacts with Ozone, as such the CLD device constitute a catalytic converter for conversion of $\mathrm{NOx}$ and $\mathrm{NO}_{2}$ to $\mathrm{NO}$. Though earlier works have also showed $\mathrm{NH}_{3}$ poisoning of $\mathrm{NO} 2$ to $\mathrm{NO}$ converter which might also lead to inaccurate NOx measurements using the chemiluminescence principle [23]. 


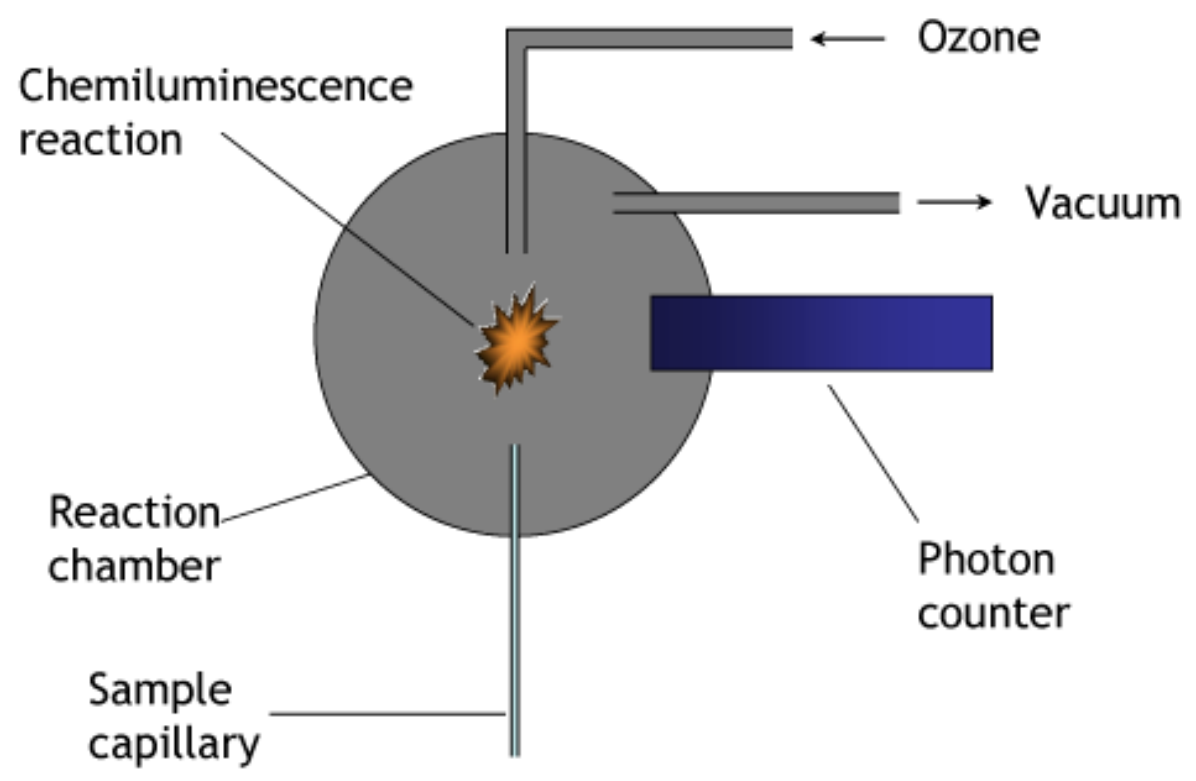

Figure 3 - Chemiluminescent Detector [24]

$\mathrm{NO}$ on reacting with ozone $\left(\mathrm{O}_{3}\right)$ produces $\mathrm{NO}_{2}$ which is in an excited state, and photon which is proportional to the concentration of the $\mathrm{NO}$ is generated when the excited $\mathrm{NO}_{2}$ molecule returns to a stable state.

$$
\mathrm{NO}+\mathrm{O}_{3}=\mathrm{NO}_{2}+\mathrm{O}_{2}+\text { Photon }
$$

The produced photon is measured by a photodetector present in the chemiluminescent detector, and the measured photon is a measure of the NO concentration.

\subsubsection{Non-Dispersive Infra-Red (NDIR) Detectors Principle}

This is a measurement principle based on infrared absorption techniques and is used for $\mathrm{CO}$ and $\mathrm{CO}_{2}$ measurement as they do have narrow wavelength with strong infra-red absorption. An infrared generator emits infrared on a chopper wheel, which filters out infrared wavelength corresponding to $\mathrm{CO}$ and $\mathrm{CO}_{2}$ narrow wavelength. A light detector then measures the intensity of the infrared light after the absorption and compares it to the intensity of the light detected for a reference cell with a gas with $0 \%$ absorption. 


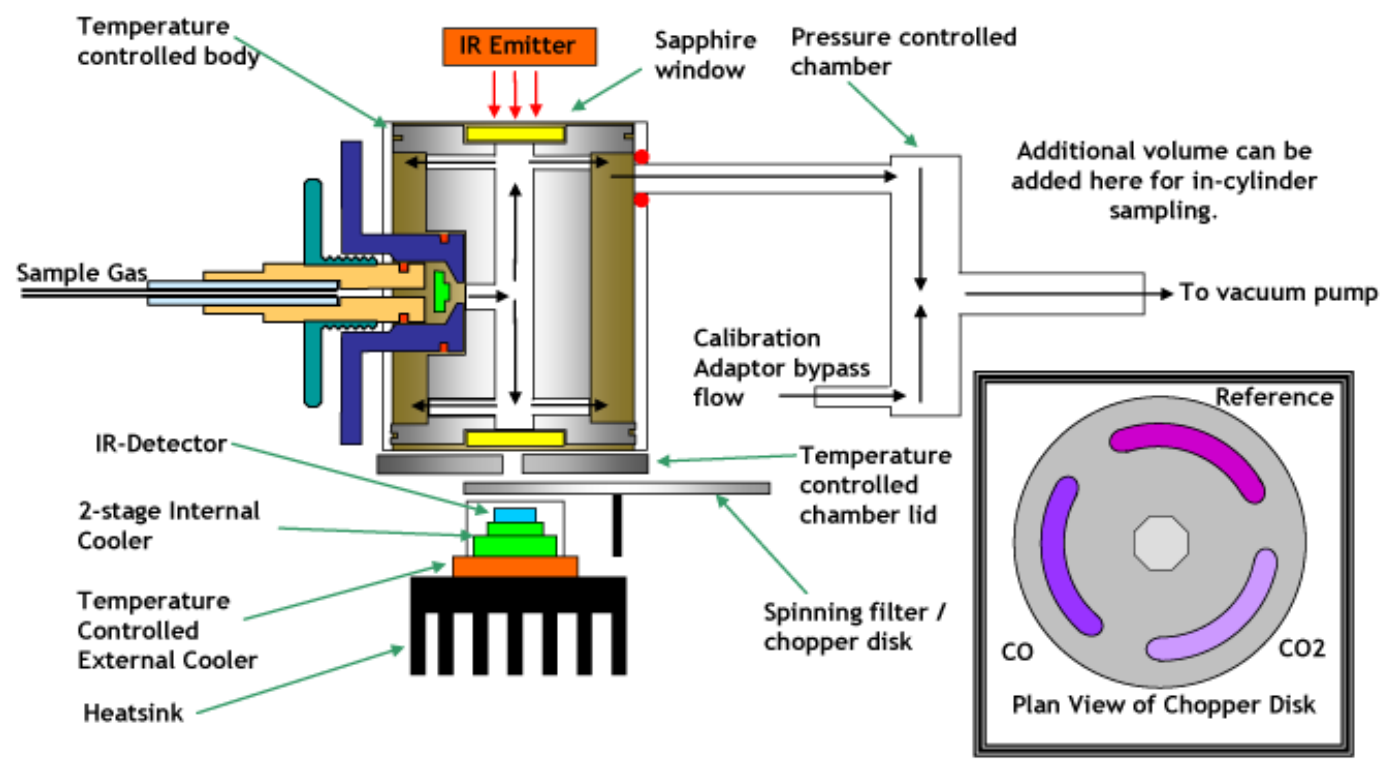

Figure 4 - NDIR Working Principle[25]

The difference between the two measured light intensities is a measure of the $\mathrm{CO}$ and $\mathrm{CO}_{2}$ concentration in the sample.

\subsubsection{Fast Flame Ionization Detector Principle}

Typically, a measurement principle used for hydrocarbons quantification using a flame ionization fuel with hydrogen and helium as its constituent. The flame ionization detector (F.I.D) fuel produce a hydrogen flame which burns hydrocarbons constituents present in any sample introduced to the FID detector. [26] 


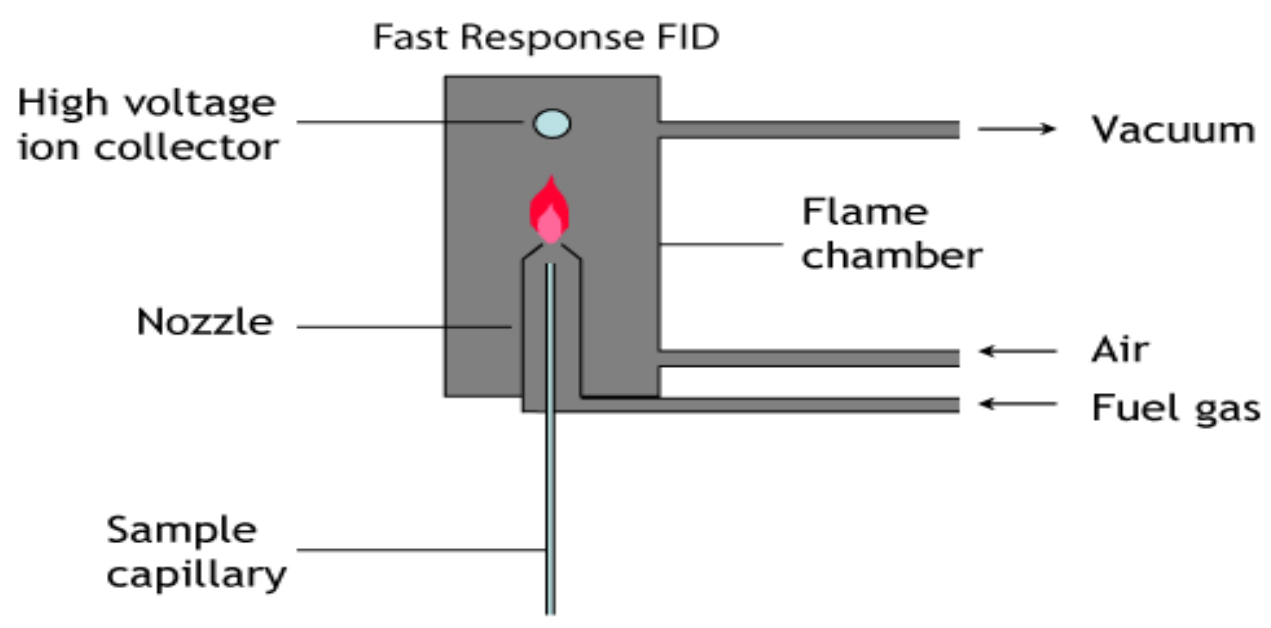

Figure 5 - Fast FID Measurement Principle [25]

The burning of THC by the FID flame generate ions which are detected with a metal collector, and the number of ions detected corresponds to the concentration of the HC present in the sample gas.

\subsection{Exhaust Gas Measuring Devices Terms}

Basic terminologies particular to gas analyzers are needful in determining the measurement application which a gas analyzer can best be suited for which are a measure of the device's limitation. These terms are performance parameters in which prior knowledge of their specification for a device aids in better judgement to decide various factors needful for consideration in the quantification and characterization process. This is evident to be useful for measurement of NOx at near zero level in which these measuring devices parameters are a major factor in ensuring the measurements are accurately carried out. Some of the common terms used includes turn-down ratio, limit of detection (LOD), response time, drift, noise, signal to noise ratio, linearity, accuracy, repeatability, resolution of instrument, selectivity, sensitivity and interference.

\subsubsection{Turn Down Ratio}

A parameter which is a measure of a device operating span within which it can accurately measure a physical quantity. It is a function of the ratio of the maximum flow which a device can measure accurately to the minimum flow which it can measure. In measurement applications a device with 
high turn-down ratio is preferable compared to one with a low turn down ratio as the former provides more measurement range across a wider range [27].

\subsubsection{Limit of Detection and Quantification}

Limit of detection (LOD) is the least concentration a measuring device can measure without necessarily being quantified accurately. There exists high level of measurement inaccuracy at the LOD range compared to the limit of quantification (LOQ) which is the lowest amount of concentration a device can measure accurately with high precision and repeatability [28].

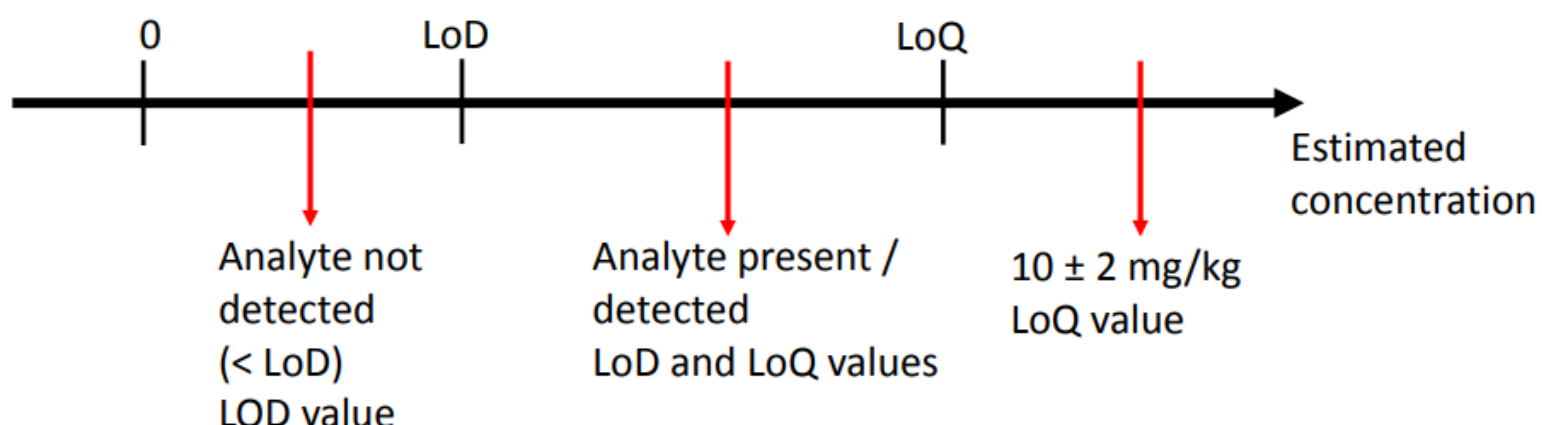

LOD value

$1 \pm 2 \mathrm{mg} / \mathrm{kg}$

$3 \pm 2 \mathrm{mg} / \mathrm{kg}$

Figure 6 - LOD and LOQ Comparison of a Measuring Device [29]

For measurement purpose it is desirable that the concentration of the emission specie to be measured should be within the limit of quantification of the measuring instrument. As the measurement inaccuracy increases when the emission specie concentration tends towards the limit of detection range as it might be detected but might not be accurately quantified.

\subsubsection{Response Time}

Response time is the time taken for a measuring device to respond and measure variations in measured readings. This is the time it takes the device to make the measured value equivalent to the actual value to be measured. For a measuring device that have attained a set-reading, at that instant it is in a sort of equilibrium, therefore any variation in reading will results in nonequilibrium therefore the instruments need time to adjust its setting and return to equilibrium which is a form of stability to be able to measure the new actual value [30]. This concurrent intermittent instability while also trying to attain measurement equilibrium is a continuous process which a 
measuring device undergoes and the ability to be able to adjust promptly in responding to variations in measurement and measure the actual value is a good measure of response time needed in a continuous emission measuring device. The EPA standards for continuous gas analyzers response time is $T_{90} \leq 10 \mathrm{~s}$. This means that the device should attain $90 \%$ of the final value to be measured within 10 seconds [31].

\subsubsection{Drift}

Drift is an overtime deviation of a measuring instrument measured values compared to the actual values. This variation in the readings of the instrument to the reference gas is a check done on instrument to determine the tendency of their measured values changing after a period of time. Drift can either be a calibration drift which is a measure of difference between the analyzers output value to a span gas value used for calibration purpose, or a zero drift in which the concentration of the reference gas to be measured is Zero [32].

\subsubsection{Noise/Signal to Noise Ratio}

Random measurement errors that leads to uncertainty in measurement accuracy of a physical quantity are called noise. This statistical uncertainty in measurement is characteristic of measuring devices which results in fluctuation of the measured value about an average value [33].

$$
\text { Data }=\text { True Signal }+ \text { Noise [34] }
$$

This parameter leads to inaccuracy as it does have significant effect by causing differences between actual value and the measured value. This phenomenon which is unavoidable comes from various factors which affects measurement [35]. The effect of noise in a measured output is usually considered and analyzed using the signal to noise ratio (SNR) which is a measure of the ratio of the true value compared to the noise levels in a given data [36]. It is most times desirable to have a high SNR value in every measurement device, as a low SNR ratio means that the noise level is of the same magnitude with the actual value measured [37].

\subsubsection{Linearity}

Deviation of a measuring instrument measured values from its ideal calibration values between zero to full input values is a measure of linearity [38]. A measuring device is set to have a relationship between its output and input, which it utilizes in giving an output value when an input is given to it. Hence, a test of linearity is how much a device does not depart from this set relationship in determining the actual output from a received input, this is usually done from $0 \%$ 
to $100 \%$ typically in an increasing manner, at a measurement value to get a curve or straight in relationship. This is then compared to the ideal straight or curved calibration relationship of the measurement system[39].

\subsubsection{Accuracy}

One of the major measuring parameters to be highly considered when selecting a measuring instrument is accuracy which is a measure of the dis-similarity between an instrument's output value to the actual value being measured. It is basically expressed as a $\pm \%$ of an instrument's calibrated span measurement or full scale measurement value [38].

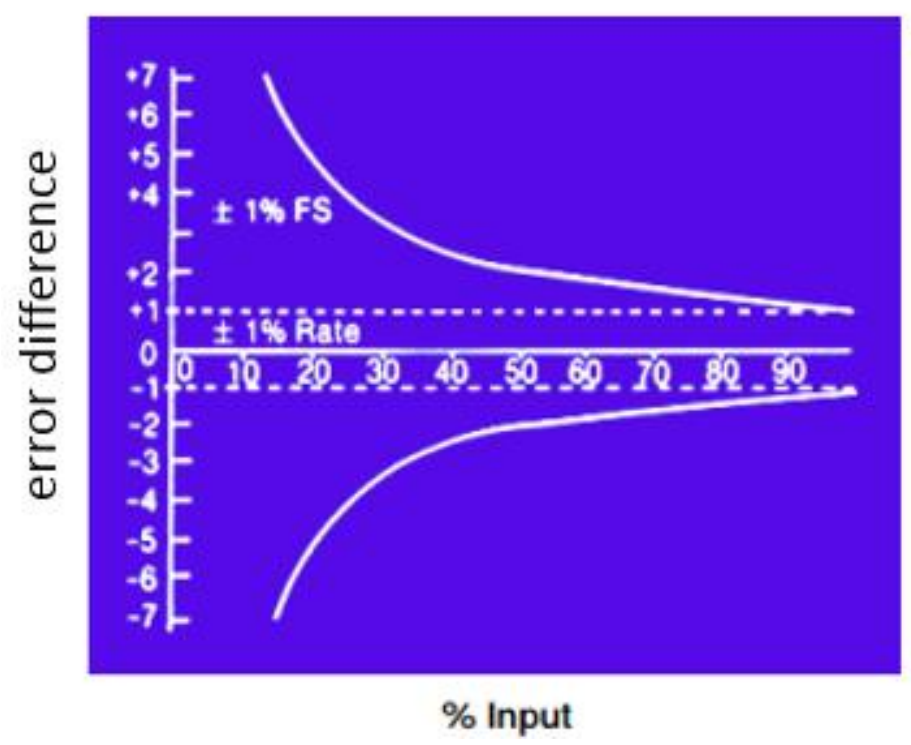

Figure 7 - Relationship Between Different Expression of Accuracy [37]

Accuracy of measuring devices can be based on absolute accuracy which is particular to the performance of an instrument, or reference accuracy which is the accuracy of an instrument working under reference conditions which is most times not obtainable in practical working condition [38].

\subsubsection{Precision}

A measurement device ability to maintain accuracy and reproduce the same values of a measurement is precision. This entails a measuring device being able to measure the value of a physical quantity multiple times and still give the same output value every time [39]. This is typically the case for an ideal sensor/measuring device but for a real sensor/measurement device 
as a result of various factors the measured value tends to change with subsequent measurements taken.

\subsubsection{Repeatability}

Property of a measuring device to precisely replicate a measurement given the same working conditions is repeatability. This specific instrument parameter has equivalent magnitude of relevance compared to the accuracy of the instrument. The accuracy of an instrument is dependent on repeatability [38].Repeatability check of a measuring instrument might involve taking repeated measured values of a physical quantity from $0-100 \%$ under the same conditions to see if the same output values are obtainable across multiple measurements.

\subsubsection{Resolution}

Resolution is the least increment that can be read on a measuring device [40]. The higher the resolution of a measuring device the more minute changes in input signal it can detect and produce corresponding minute output changes. This parameter gives devices ability to produce readings with high level of accuracy as minute changes can be easily detected. The resolution of a device is specified either as a percent of its full-scale measurement, measured value or in absolute terms [39].

\subsubsection{Sensitivity}

Sensitivity is a measure of the minimum input that can be applied to a measuring device to cause a change in the output value. While resolution is more of the least output value, sensitivity is the least input that can be noticed by the measuring device to cause a change in the output value. A least amount of change in a measured quantity input which the measuring instrument can detect and produce an output.

\subsubsection{Interference}

Interference is the effect of other physical quantity in the accuracy of measuring a specified physical quantity. Interference affects measurement by either increasing or decreasing the measured value such that instrument is unable to measure a predefined physical quantity accurately. The compound being sampled to quantify the concentration of a target gas do contain other substances which their presence affects the reading of the target gas. This can be either through reactivity (reduction, or oxidation) reactions or inhibition between the target gas and constituents' gas, thereby increasing, decreasing or annulling the concentration of the target gas. 


\subsubsection{Cross-Sensitivity}

Cross-sensitivity is a form of interference in which a measuring device is sensitive to multiple substances or quantities. This results in the measuring device measuring the target quantity alongside other quantities which leads to measurements error. Cross-Sensitivity is more on the measuring device not being specific enough by measuring multiple quantities[41].

\subsubsection{Selectivity}

Selectivity is a measure of specificity of how much a measuring device can only measure a specific quantity without interference from other substances present. A measuring device with high selectivity will be specific in measuring a target quantity with minimal interference from other quantity. $100 \%$ selectivity connotes a gas analyzer being selective towards only the target gas amongst other similar compounds present.

\subsubsection{Calibration}

This is a test carried out for proper operation of an analyzer. It is a check used to determine the relationship between input and output values of a device. This is a practical means of converting undefined signals to engineering units which measuring devices relies on and utilize in giving corresponding accurate output response to a given input. This check is done occasionally to establish the authenticity of a measured readings, since accurate measurement of an input is dependent on calibration [42].

\subsubsection{Linearization}

This is a check on measuring devices to determine if they produce proportional and accurate readings over a graduated measurement span. This is achieved using a minimum of 10 set-points of known values usually a reference value to verify the accuracy and proportionality of the measurements [43]. The setpoints can be from $0-100 \%$ of a set (Span) value and the values at each $10 \%$ increase is checked and at the $100 \%$ set-point the value is decreased in steps of $10 \%$ to the $0 \%$ scale. The values gotten can then be analyzed for linearity, accuracy and proportionality across each set-point.

\subsection{Emission Measurement and Sampling System}

Emission measurement entails all process involved in the characterization and quantification of the constituents of an exhaust gas. There are standard requirements as per Code of Federal 
Regulations (CFR), Title 40, Part 1065 (USEPA). This is to ensure accuracy and uniformity in the measurement process across all laboratory or engine test cell and field testing used for certification, emission testing and research purpose. The exhaust gas from automobiles must be conditioned to meet the capability of the measuring instrument in terms of temperature, concentration, water content, to ensure the devices are suitable to measure these exhaust gas accurately in this condition. This process of adjusting the properties of the exhaust gas is termed sampling and is needful to ensure the chemical properties of the exhaust gas to be sampled does not change neither the exhaust gas constituents lost between the exhaust point and the measurement point [44]. Exhaust emission can be sampled either raw or diluted. The diluted sampling is the standard process adopted due to its highly standardized measurement procedures and equipment used which gives high accuracy, precision and repeatability. This is typical of the CVS measurement system which utilizes the full flow dilution tunnel compared to the raw sampling system which is adopted in portable emission measurement system (PEMS), which is used in real world testing and has multi-variable conditions making standards measurement procedures difficult to achieve and as such limits repeatability amongst other measurement integrity checks [45].

\subsubsection{Raw/Direct Exhaust Sampling}

Measurement of emissions directly from the vehicle exhaust without diluting is referred to as raw sampling, which is the typical of PEMS on-road testing. This is mostly used for field testing and the emission constituents usually have higher concentrations and temperature compared to the diluted exhaust sampling system emission constituents.

\subsubsection{Dilute Exhaust Sampling System}

Dilute exhaust sampling system is a process in which raw exhaust is diluted before measurement. This avoids condensation of the raw exhaust gas by increasing the partial pressure and as such reduces possibility of sample condensation. It typically offers more sample available for measurement while also conditioning the sample. This reduces the sample's concentration and temperature or dries it to suit the measuring capability and principle of the measuring instrument. The most common dilute exhaust sampling system are full flow dilution tunnel (CVS) System and the Partial flow dilution system. The main difference between the two sampling systems is that the full flow dilution system dilutes the whole exhaust gas with air. While the partial dilution flow dilutes only a portion of the exhaust gas extracted from the exhaust sample with air. The CVS 
system is widely more acceptable for regulatory purposes with lesser usage limitations compared to the partial flow dilution system [46].

\subsection{Constant Volume Sampling Measurement System}

This is a set-up which is used in the measurement of emission species by typically diluting the total exhaust with air, while also maintaining a constant flow rate to ensure each emission specie can be quantified based on the measured flowrate. This is the adopted standardized emission measurement in the U.S for certification and regulatory purposes due to its high-level of accuracy [47].

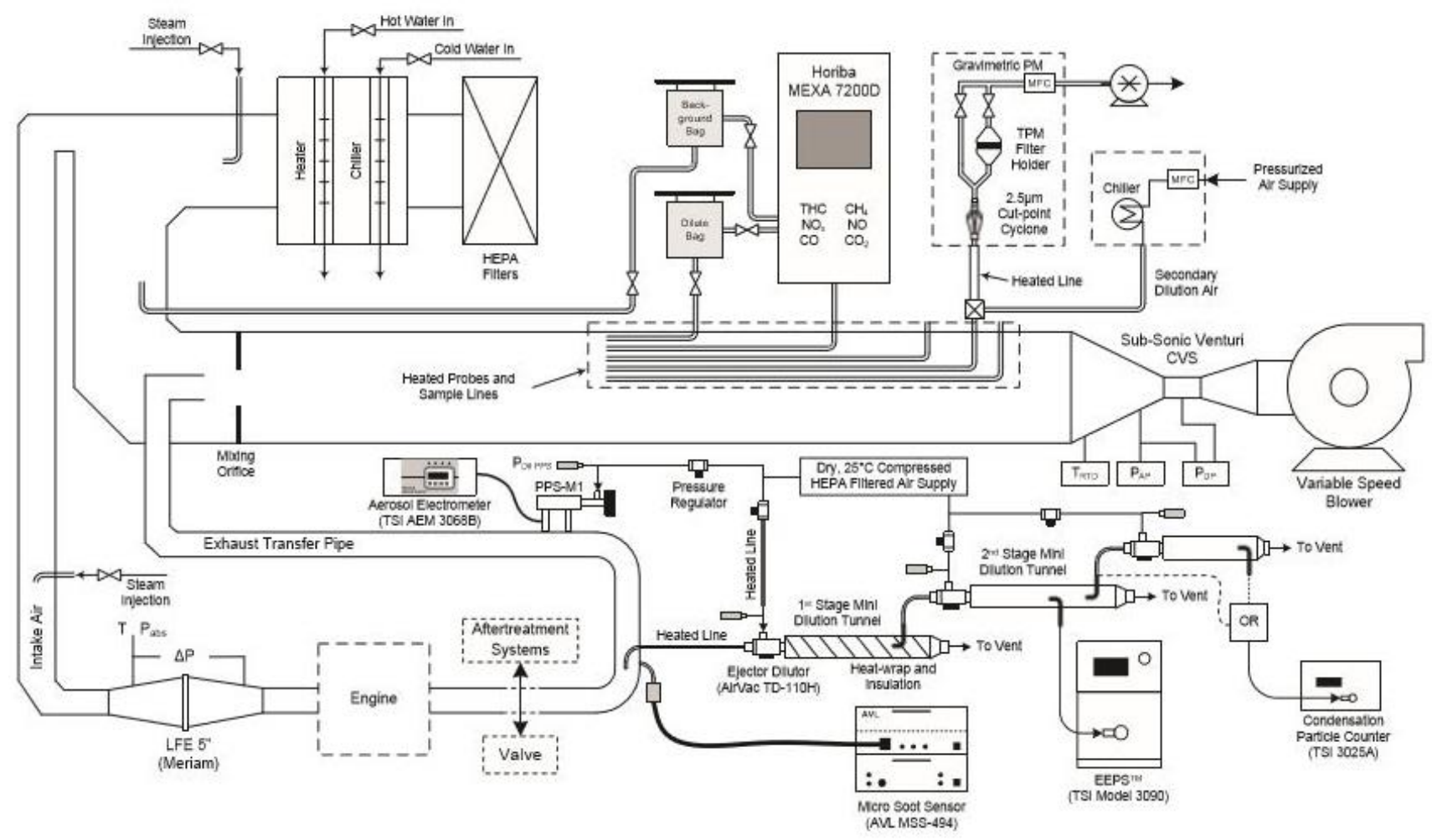

Figure 8- Overview of the CVS Measurement System Set-up[48]

It is also referred to as full flow dilution since the whole exhaust is diluted and uniformly mixed before some of the portion is sampled. The concentrations of the constituent of the sampled exhaust gas is calculated using the measured constant flow rate. The measured flow rate is the sum of the exhaust flow rate and the dilution air flow rate which the constant volume sampling system ensures to be at a constant flow rate. The dilution air reduces the temperature of the exhaust gas and the concentrations, while also avoiding condensations of the sample to avoid loss as the hot exhaust gas has water vapor as its constituent and on contacting a cold spot along the measuring 
system the water condenses. Some samples are lost in the condensed water as some of the exhaust specie are soluble in water while some of the exhaust constituents might end up hanging on the walls of the emission sampling system. This is typically avoided by heating up the sampling system or conditioning the system to ensure the emission species remains the same from the exhaust to the sampling point. Apart from the aforementioned reasons, the dilution also helps to condition the exhaust gas for easy measurement at the sampling port. This avoids variability or change of the physical or chemical properties of the sampled exhaust between the engine exhaust port and the measuring system port. The conventional CVS measurement system has two tunnels which are the primary and secondary dilution tunnel, in which the secondary tunnel is used for PM sampling and measurement while the primary dilution tunnel is used for the measurement of other exhaust sampling asides the PM measurement. The CVS set-up is done in the U.S according to the EPA 40 code of federal regulations CFR) 1065 standards, which is a monitored and controlled system of measurement. The material of the components used in the sampling process should have no chemical affinity for the exhaust gas, to avoid chemical reaction with the emission species as this may alter the specie during the measurement [47].

\subsubsection{Components of a CVS Measurement Set-up}

The major part of the set-up is a Constant Volume Sampler (CVS) which maintains a constant flow rate all through the sampling process to aid in the calculation of the emissions specie at the end of the measuring process. The CVS set-up has various sub-system which includes: exhaust sampling and flow measurement system, background sampling and flow measurement system and the dilution tunnel. A typical CVS measurement set-up has various systems and can be categorized based on this study to 4 sub-systems which are: [47]

1. Exhaust Dilution System

2. Analytical Sampling System

3. Operating Systems and Data Acquisition (D.A.Q)

\subsubsection{Exhaust Dilution System}

Exhaust dilution system is where the exhaust gas is diluted by mixing it with ambient air. The mixing plane is where the engine out emissions from the exhaust mixes with the dilution air. The diluted exhaust gas is passed through a CVS tunnel which has a length 10times the diameter of the tunnel to ensure the exhaust gas and dilution air is well-mixed, for a homogeneous mixture before the sampling port. 


\subsubsection{Analytical Sampling System}

Sampling port and sampling system which could be specified for either the background or exhaust measurement system is characteristic of this portion of the CVS sub-system, as the exhaust is sampled for measurement at this section. The background sample port is where the intake air (dilution air) is collected while the exhaust sample port is where the homogenous dilute exhaust gas is collected. The exhaust and background sampling system connect the extracted exhaust and background sample respectively to the analyzer system where it will be quantified. The analyzer system could be a bagging system which is designed to take average emission values over a time period, or a continuous sampling system which takes instantaneous emission values. The total measured emission value taken by a bagging system which is averaged overtime is a representative of the total value of the overtime continuous sampling system [49].

\subsubsection{Operating Systems and Data Acquisition (D.A.Q)}

These are systems used to control, monitor and log data from the CVS set-up. This system is an integration of various subsystems which might include sensors mounted along the set-up which gives feedback in form of signals, which is interpreted based on calibration [21]. The analyzers measurements are sent to a central system likewise other signal output which is processed and stored by this data collection system. While also processing the feedbacks and inputs from sensors for monitoring and automating the control. To adjust the emission measurement conditions and take decisions based on set commands [50].

\subsubsection{CVS System Calibration and Verification}

These are various test carried out based on "EPA 40 CFR 1065" procedures on a CVS measurement set-up to ensure integrity of the CVS system and to ensure the measuring systems and devices can accurately capture various parameters and physical quantity measured. As these parameters are necessary in emission quantification and characterization. 
Table 3 - Calibration Data and Measurement Tolerance for CVS Set-up [51]

\begin{tabular}{|c|c|c|}
\hline Parameter & Units & Sensor-readout Tolerances \\
\hline Barometric Pressure (Corrected) & $\mathrm{kPa}$ & $\pm .340 \mathrm{kPa}$ \\
\hline Ambient Temperature & ${ }^{\circ} \mathrm{C}$ & $\pm .28{ }^{\circ} \mathrm{C}$ \\
\hline Air Temperature into Metering Venturi & ${ }^{\circ} \mathrm{C}$ & $\pm 1.11^{\circ} \mathrm{C}$. \\
\hline $\begin{array}{l}\text { Pressure drop between the inlet and throat of } \\
\text { metering Venturi }\end{array}$ & $\mathrm{kPa}$ & $\pm 0.012 \mathrm{kPa}$ \\
\hline Air Flow & $\mathrm{m}^{3} / \mathrm{min}$ & \pm 0.5 percent of NIST value. \\
\hline Air temperature at CVS pump inlet & ${ }^{\circ} \mathrm{C}$ & $\pm 1.11^{\circ} \mathrm{C}$ \\
\hline Pressure depression at CVS pump outlet & $\mathrm{kPa}$ & $\pm 0.055 \mathrm{kPa}$ \\
\hline Pressure head at CVS pump outlet & $\mathrm{kPa}$ & $\pm 1.11^{\circ} \mathrm{C}$ \\
\hline Pump revolutions during test period & Revs & \pm 1 Rev. \\
\hline Elapsed time for test period & $\mathrm{s}$ & $\pm 0.5 \mathrm{~s}$ \\
\hline $\begin{array}{c}\text { Air temperature at CVS pump outlet } \\
\text { (optional) }\end{array}$ & ${ }^{\circ} \mathrm{C}$ & $\pm 1.11^{\circ} \mathrm{C}$. \\
\hline
\end{tabular}

Accurate flowmeter and restrictor valve are used to calibrate the CVS system according to the EPA standards. This is to ensure the flowrate and other parameters of the CVS is accurately measured during testing. Some of the measured parameters includes barometric pressure, ambient temperature, venturi-inlet air temperature and pressure drop across venturi. The restrictor valve is adjusted, while the measurement are taken to get data points for the calibration of the CVS set-up [51].The verification of the CVS system set-up is done to check the integrity of the measurement system. This is a means to determine that the whole CVS system set-up including the gas analyzers have the capability to accurately quantify a measured gas. This is also a measure to perform leak test of the system. The verification test carried out is propane injection, in which a known mass of propane is injected into the set-up and measured at the analyzers end. The amount of recovered propane is calculated using the measured values at the gas analyzers, this is compare to the injected mass and according to the " 40 CFR 1065" standards it must be within $\pm 2 \%$ [52]. The CVS system passing the propane check invariably certifies to an extent correct analyzer calibration, leak-proof, efficient mixing, non-contaminated sample System, accurate CVS calibration, accurate flow meter measurements and accurate CVS data acquisition devices. In which fault in any of the aforementioned can lead to great error and thereby cause the verification process to fail [52]. Propane under-recovery can be as a result of cold spots on the CVS tunnel, or possibly due to leaks in the line supplying the sample gas to the analyzer. Over-recovery is another major challenge which could be due to background contamination and flow measurement issues resulting from leaks after the sampling plane. 


\subsection{Challenges of the CVS for Emissions Measurement}

The CVS set-up has inherent challenges associated with its use for measuring emission species, this is as a result of its operating principles. Some of the common challenges is time alignment in data recorded as there is always time difference between when emission is measured and when the emission actually leaves the Engine's EATS [53]. There is also high possibility of condensation of water vapor from the exhaust in the CVS tunnel during measurement which can result due to improper dilution ratio. Cold spots occurrence due to this effect can result in the loss of some emission specie which are soluble in water, while also increasing the chances of having hang-ups in the CVS sampling measurement system set-up [53]. Another challenge has been attributed to interferences from constituents of the ambient air used in the dilution of the exhaust sample[53].The ambient air also exhibits some sort of variability which affects the measurement of emission specie such as NOx and PM [54].The variability in ambient air constituent's concentration is also not accounted for , as the ambient air is assumed to have a constant concentration all through the emission sampling period which is actually not the case [55]. There has also been the case of measurement errors attributed to devices used in the CVS measurement set-up. This might result in inaccurate readings of measured flowrate, pressure, temperature, humidity and other sensors leading to emission quantification errors [56] as these values are cogent in accurate calculation and processing of emission data.

\subsection{Limitation of the CVS System in NOx Measurement}

The CVS sampling system though has its general intrinsic measurement challenges there are some challenges specific to the use of the CVS measurement set-up for NOx measurement. A major drawback in CVS for NOx measurements is the loss of NOx in the CVS system. This results in the CVS set-up not being able to attain the below $\pm 2 \%$ NOx loss recovery check passing criteria according to the "EPA 40 CFR 1065" standard [57].The losses is attributed to various factors which includes diffusion of NOx into silicone hose, hang -up of NOx on CVS tunnel wall due to cold spots, condensation of water vapor in the exhaust gas which results in the loss of NOx due to its solubility in water [58].The analyzers used in the CVS set-up for NOx measurement has inherent measurement errors associated with "limitations due to their specification value" such as drift, limit of detection, interference with other gases such as $\mathrm{NH}_{3}$ and $\mathrm{CO}_{2}$, high cross-sensitivity ,low selectivity and high response time amongst other parameters which results in high error in accurately quantifying the emissions [56]. 


\subsection{Challenges with NOx measurement at Near Zero Level}

Earlier works have attached difficulties to quantifying and characterizing NOx at low levels as the measurement of NOx at this level has its intrinsic challenges particular to it [54]. As emission measurements at this low level requires higher degree of accuracy, precision and minimal error. The effects of these aforementioned parameters become more significant due to the low level at which quantification is performed. When measurement of NOx goes below 50ppm it gets difficult to measure and the measurement error and inaccuracy increases as the measured values tends towards zero $(<10 \mathrm{ppm})$ range [1].

Table 4 - Error Variation with Decreasing Concentration [1]

\begin{tabular}{|c|c|}
\hline Measurement Readings Maximum $(\mathrm{ppm})$ & Percent of Readings > Maximum(ppm) \\
\hline 10 & 21.5 \\
\hline 8 & 27.3 \\
\hline 6 & 40.2 \\
\hline 4 & 35.2 \\
\hline 2 & 51.8 \\
\hline
\end{tabular}

The devices currently available in quantifying emissions have their measurement limitations which includes having higher limits of detection compared to the current emission trends. This poses difficulty in capturing and accurately measuring at these near zero emissions level, as these values are close to the instrument's noise level thresholds. Emission measurement devices are prone to drifting overtime and drifting at this low emission levels also affects correct characterization and quantification of emission species. Some of the difficulties are also attributed to the limitation of the current NOx analyzers, such as the drifting of the analyzers, delay in settling back to zero range after a brief Spike. Interferences from other gases such as $\mathrm{HC}$ and $\mathrm{NH}_{3}$, calibration gases concentration limits, hang-up of NO/other contaminants in the analyzers/CVS system set-up and the condition of the CVS system adds to this inaccuracy. CVS set-up conditions such as dilution ratio is also crucial when quantifying NOx at near Zero level as measuring devices are reaching their detection limit to accurately measure NOx accurately. The error associated with small difference between background and the exhaust gas to be measured when diluted has increased difficulty in quantification. The dilution ratio of the raw exhaust has to be optimized to ensure the concentrations are high enough while also avoiding condensation of the exhaust sample due to insufficient dilution ratio. 
The effect of quenching due to $\mathrm{CO}_{2}$ and water vapor on NOx measurement using the CLD is very significant at near zero [57]. Third body quenching effect which is due to the interaction of NO with other substances during CLD measurement decreases the relative quenching which is the ratio of NO measurement without other gas interaction compared to when they are present [59].

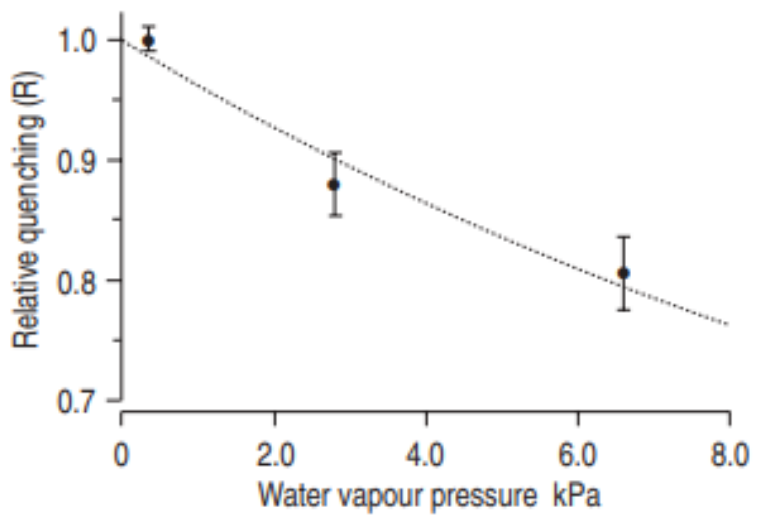

Figure 9 - Effect of Water Vapor on Relative Quenching of NO [59]

This depicts that the increasing concentration of water vapor or $\mathrm{CO}_{2}$ in the NO sample will lead to reduced values gotten. Critical analysis of the above showcased that if the concentration of the water vapor is left constant and at higher NOx measurement the relative quenching effect is 0.95. But as soon as the concentration of the measured NO gets lower the percentage of the water concentration present in the NO sample becomes significant, and therefore have a greater effect.

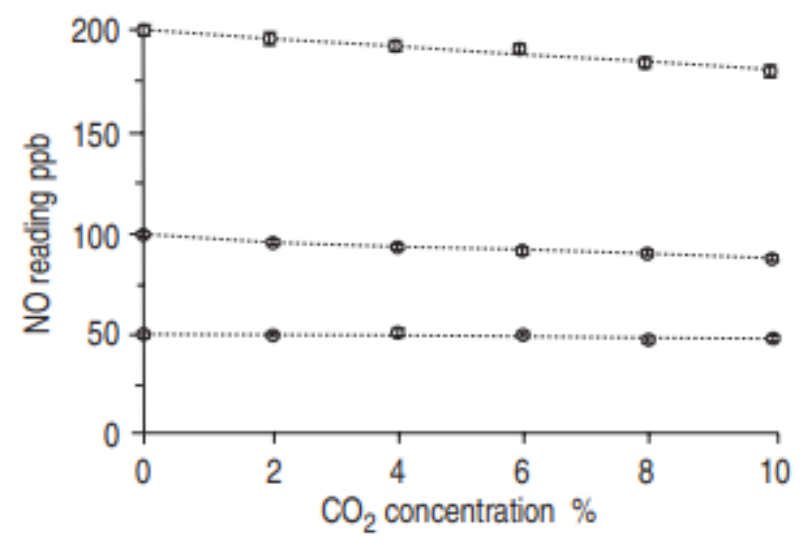

Figure 10 - Effect of $\mathrm{CO}_{2}$ Concentration on Measured NO Value [59]

At higher concentration of $\mathrm{CO}_{2}$ the same effect observed due to relative quenching of $\mathrm{NO}$ as a result of water vapour will also be experienced, but this effect gets significant at near zero level. 


\subsection{Enhancement of the CVS system Set-Up for Accurate Low NOx Measurement}

Earlier works has identified need for modifications to the current CVS measurement set-up and techniques for accurate NOx measurement at low level. One of such is incorporating a proportional background sampling to cater for variability in background air [60]. According to a recent work analyzer with lower measurement range, low response time, unbiased reading, minimal quenching and drifting effect according to "EPA 40 CFR 1065" standards should be used. While also ensuring minimal analyzer interference, enhanced monitoring, control and measurement of flow coupled with better control of Water [60].

Dilution air filtration system is also proposed to ensure consistent ambient air concentration thereby reducing variability overtime in the measured NOx [54]. A virtual NOx injection was also carried out in earlier works to determine the sections of CVS system with much losses. Hence, possibly attribute it to a cause and effect relationship, while also looking at possible measures to curb or eliminate these losses [57]. Heating of the dilution air and CVS tunnel is also a measure to reduce loss of NOx as the NO to be measured will remain in gas phase. This increase in temperature therefore reduces or probably eliminate the possibility of the NO gas getting attached to the tunnel walls, while also maintaining the sample above the dew point to avoid water condensation in the CVS sampling system set-up [58]. Connection lines are also proposed to be made shorter to reduce assumed NOx loss in the sample lines [53] which also reduce residence time before measurement is carried out [58]. 


\section{Experimental Set-up and Procedures}

Overview of the experimental set-up used for this study is detailed here. The test cell set-up, measurement procedures and devices used coupled with a brief outline of the test cell used is described herein.

\subsection{Testing Laboratory}

The testing and data collection for this work was done at the Vehicle Emission and Testing Laboratory (VETL) which is part of the Laboratory facilities of the Center for Alternative Fuels, Engines and Emission (CAFEE) of West Virginia University (WVU). The study was conducted following the specified standards according to the Code of Federal Regulations (CFR), Title 40, Part 1065 (USEPA).

\subsection{Overview of the General Test Set-Up}

The general set-up is comprised of an engine test cell in which the engine used for emission sampling was mounted and controlled to give the desired output. This engine was coupled with an after-treatment system to reduce the engine out NOx emission to the near zero level range, which is the basis of this study. The engine test cell emissions from the exhaust was directed to the CVS system for sampling under well controlled, monitored and conditioned environment following set standard. While ensuring the measurement systems and procedure were representative of what is obtainable according to emission regulations. Some specific sections of the general set-up were used for some of the study, in which some parts of the set-up were excluded. More emphasis is laid in subsequent sections of this report on the specific portions of the general set-up used for each study.

\subsubsection{Engine Test Cell}

The Engine Test cell had a 2017 D13 engine mounted for this study. An after-treatment system which will ensure low exhaust tail pipe NOx emission to ensure meeting the current emission standards was coupled with the engine in the engine test cell. The EATS comprised of SCR, DPF and DOC which was utilized coupled with and EGR emission reduction strategy. 
Table 5 - Specifications Test Engine

\begin{tabular}{|l|l|}
\hline Model Year & $\mathbf{2 0 1 7}$ \\
\hline OEM & Volvo \\
\hline Model & D13 \\
\hline Fuel & Ultra Low Sulfur Diesel \\
\hline Displacement (Liters) & 12.8 \\
\hline Bore and Stroke & $131 \times 158 \mathrm{~mm}$ \\
\hline Compression Ratio & $17.0: 1$ \\
\hline Fuel System & Common rail fuel injection \\
\hline Torque-Range & $1450-1850 \mathrm{lb}-\mathrm{ft}$ \\
\hline Rated Speed & $1375 \mathrm{RPM}$ \\
\hline Rated Horse-Power (HP) & $375-500 \mathrm{HP}$ \\
\hline Emission After-Treatment System & EGR+DOC+DPF+SCR \\
\hline NOx Emission Certification & $0.2 \mathrm{~g} / \mathrm{bhp}-\mathrm{hr}$ \\
\hline
\end{tabular}

The engine used which has its specifications tabulated in the Table 5 was connected to the DAQ system to broadcast its operating conditions and provide a means to control the engine using some computer software interface. 


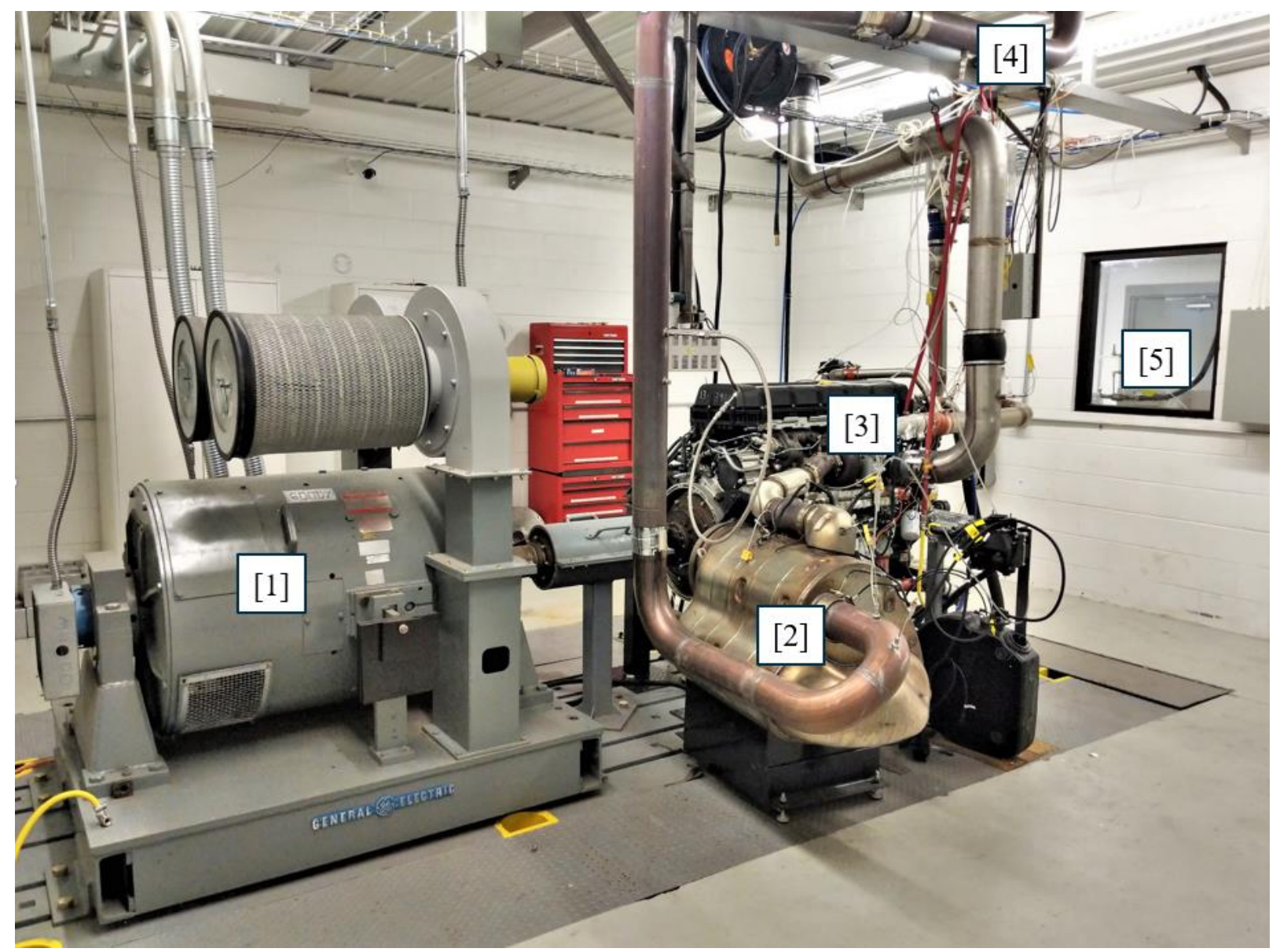

Figure 11 -Engine Test Cell: Dyno [1], EATS [2],2015 DD15 engine [3], Connection to CVS [4], Control room [5]

\subsubsection{Exhaust Sampling System (CVS)}

Full flow dilution sampling system was used for this study in which the whole exhaust from the engine test cell is diluted using ambient air and the homogenous mixture created in the CVS tunnel after mixing in the mixing plane is sampled at the sampling port and supplied to the analyzers which measured the concentrations of each gas constituents. The overview of the emission sampling system used is shown in Figure 12 which was according to the EPA $40 \mathrm{CFR}$ 1065 standard. 


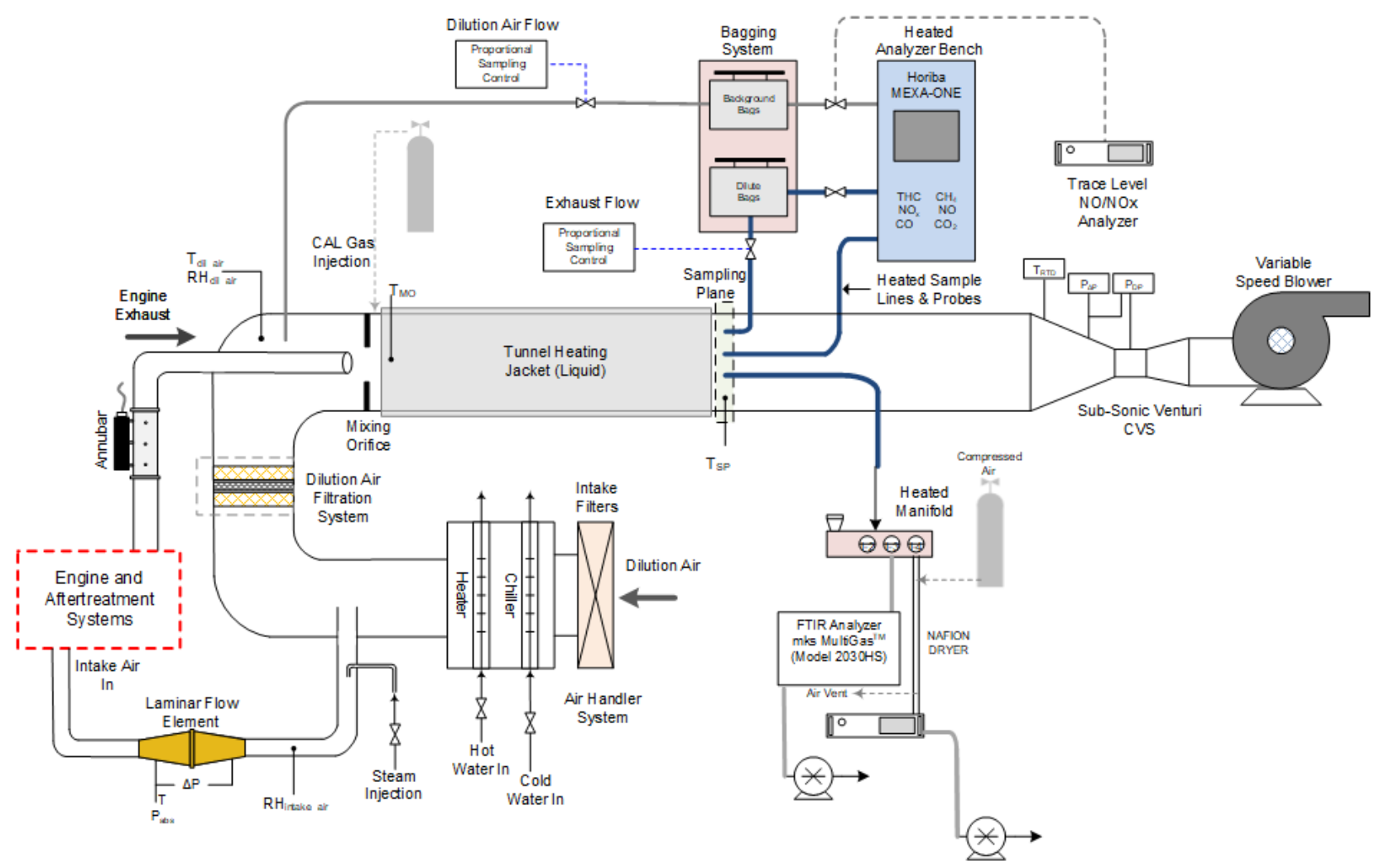

Figure 12 - Schematic of the Emission Sampling Set-up adapted from [48]

For this study NOx measurement at low level was the primary focus, as such the gas analyzers selected were specific for the measurement of NOx with high accuracy at near zero level. Multiple analyzers were also used for the purpose of comparison and measurement alignment.

\subsection{List of Devices Used}

Modifications made to the conventional CVS set-up and sampling system used in the VETL laboratory for the purpose of this study will be the emphasis of this section. These improvements were made according to recommendations from earlier studies and these were needful to adapt the CVS system for this near zero accurate NOx measurements. The enhancements include utilizing low NOx analyzers for measurements and dilution air filtration system to reduce variability and maintain consistent intake air concentration.

\subsubsection{Low NOx Analyzers}

Typically, these are trace level NOx analyzers suited with capabilities to measure NOx at low ranges. These includes the Brand-gauss and Eco-Physics NOx analyzers which were used alongside Horiba MEXA One - CVS and MKS FTIR 2030 for measuring NOx emissions from the engine exhaust diluted in the CVS set-up. The Brand-Gauss 7705 NOx Analyzer measures total 
NOx using the CLD measurement technique coupled with a high temperature $\mathrm{NO}_{2}$ converter. The specifications included $0.1 \mathrm{ppm}$ zero drift, noise level of $0.02 \mathrm{ppm}$, measurement range of $0-50 \mathrm{ppm}$ with a $T_{95}<15$ seconds and $T_{50}<7$ seconds. The ECO-Physics CLD 64 NOx analyzer had a measurement range of $100 \mathrm{ppm}$ with a minimum detection limit of $2 \mathrm{ppb}$ and $T_{50}<1$ seconds.

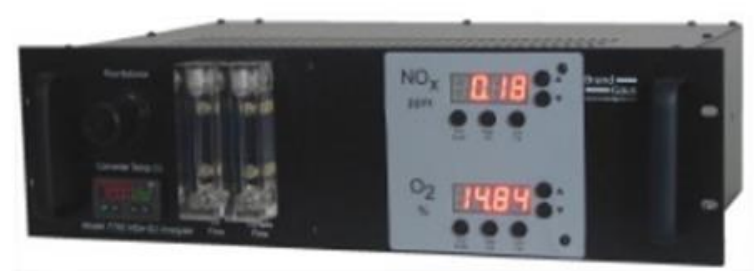

[1]

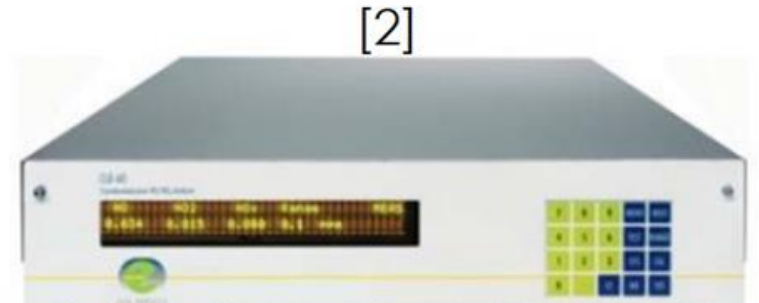

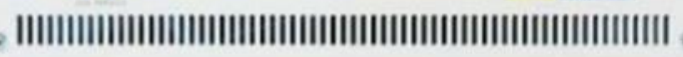
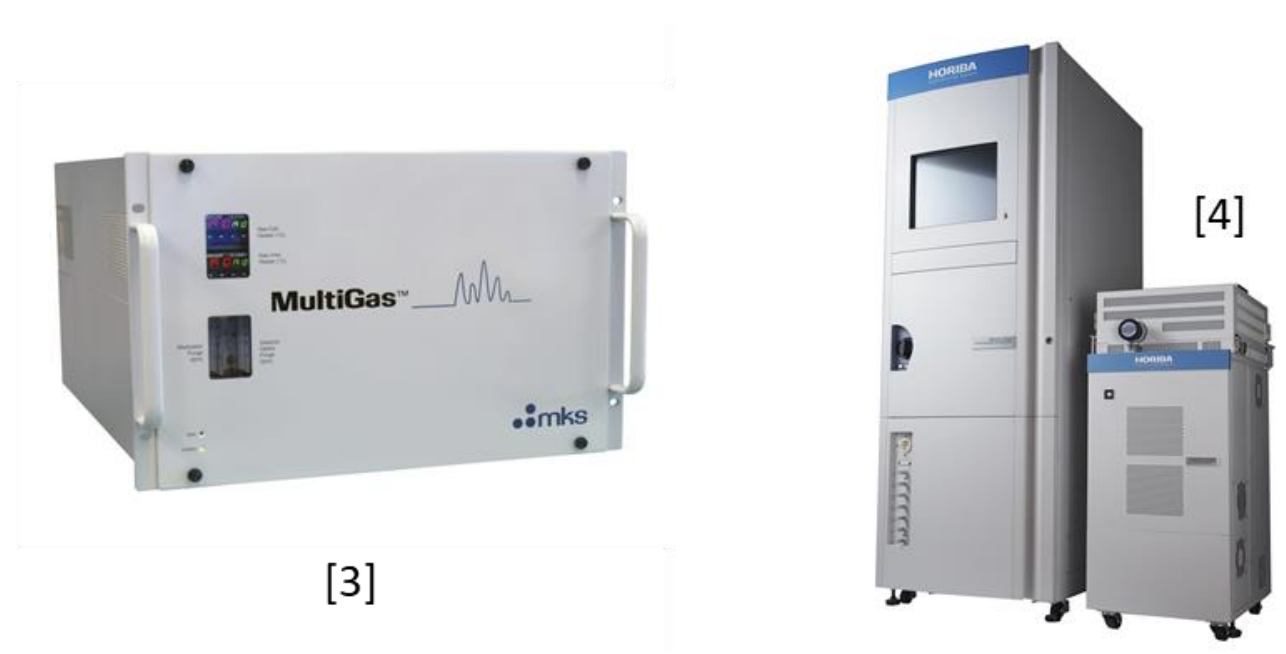

Figure 13- Trace level NOx analyzers: Brandgaus 7705[1], Ecophysics CLD64 [2], MKS FTIR 2030 [3], Horiba MEXA-ONE [4]

\subsubsection{Dilution Air Filter}

This is an activated charcoal-based filter obtained from Air filters, Inc. The filter model is a ULPA V-cell filter and based on usage in earlier studies, this filter showcased being effective in reducing gases such as NOx, THC, Volatile Organic Carbon (V.O.C) and SOx in the dilution air. 

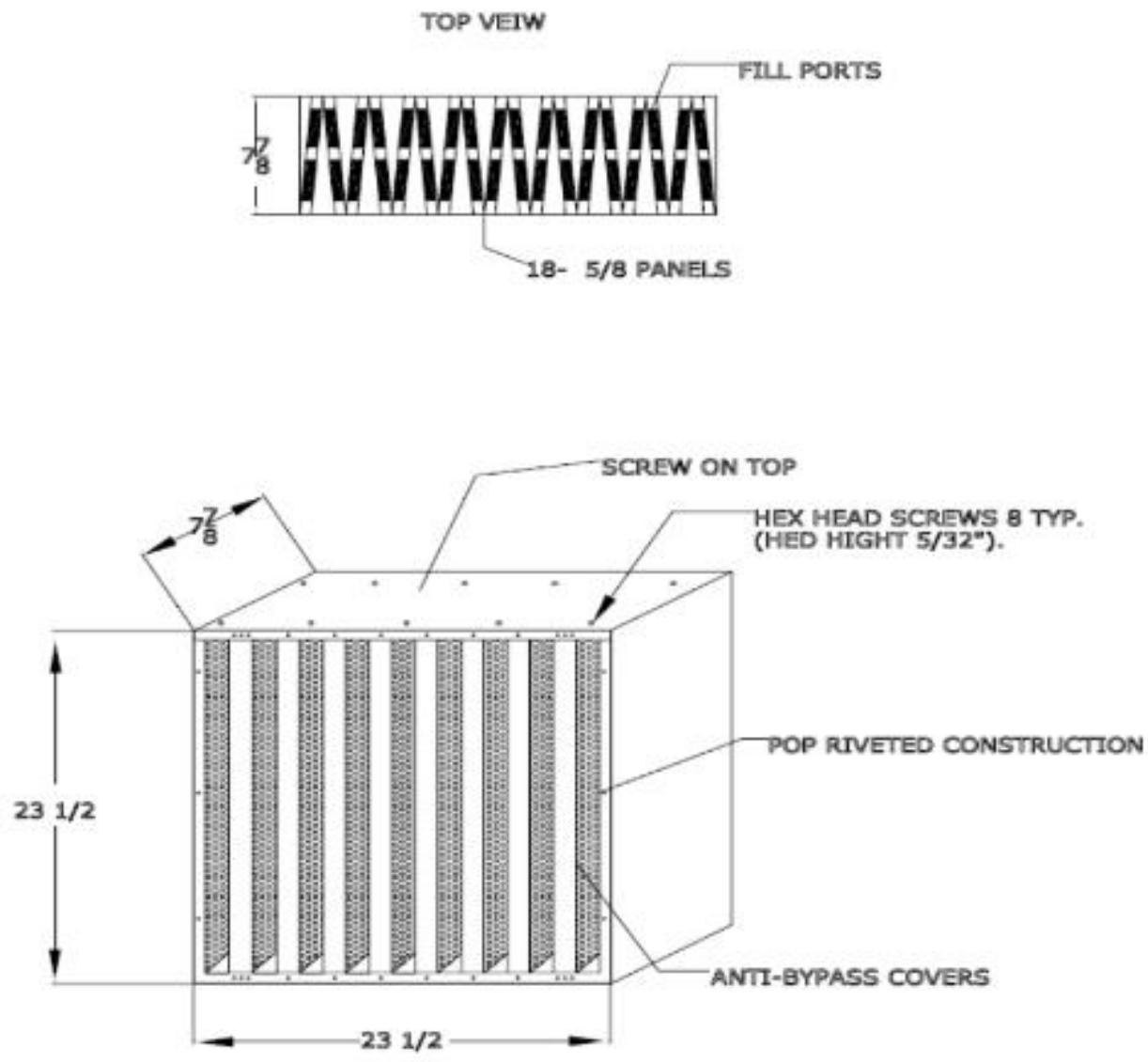

Figure 14 - Schematic of the Dilution Air Filter [61].

The filter model has a Minimum efficiency reporting value (MERV) of $\mathrm{EN}_{1822}-\mathrm{U}_{15}$. This infers having a capability of reducing high NOx levels in ambient air to the $0.1 \mathrm{ppm}$ range levels while also reducing the level of $\mathrm{HC}$ such as non-methane hydro-carbon (NMHC) concentrations in the dilution air. This ensure the dilution air attains a consistent concentration value all through the measurement process. Reduction of the hydrocarbon concentrations is critical quenching effect caused by hydrocarbon interferences in CLD NOx analyzers is quite significant in accurately measuring NOx at low level [59].

\subsubsection{Proportional Bag Sampling System}

This was incorporated to enhance the CVS measurement system by ensuring corresponding background and dilution air sampling is varied at all instance thereby accounting for any variations in concentrations over measurement duration. This typically ensures the error due to background correction is reduced or eliminated as previous studies shows variations in the background NOx 
concentration with time. The dilute bag was filled proportionally to the exhaust flow while the background bag was filled proportional to the dilution air flow.

As a result, the assumption of a constant ambient concentration is refuted, and necessary adjustment were made using mass flow controllers which ensure that the ratio of the background air mass is proportional at all instance to the mass of the dilution air to be able to cater for variability in the concentration of the ambient air. Emission samples for the Ecophysics and Brandgauss NOx analyzers was dried using Nafion tubes. The Mexa analyzer measured emission as dry samples after the water was dropped using chiller while the FTIR measured samples wet. Dry to wet corrections was performed before analysis of the emission measurements from the different analyzers used.

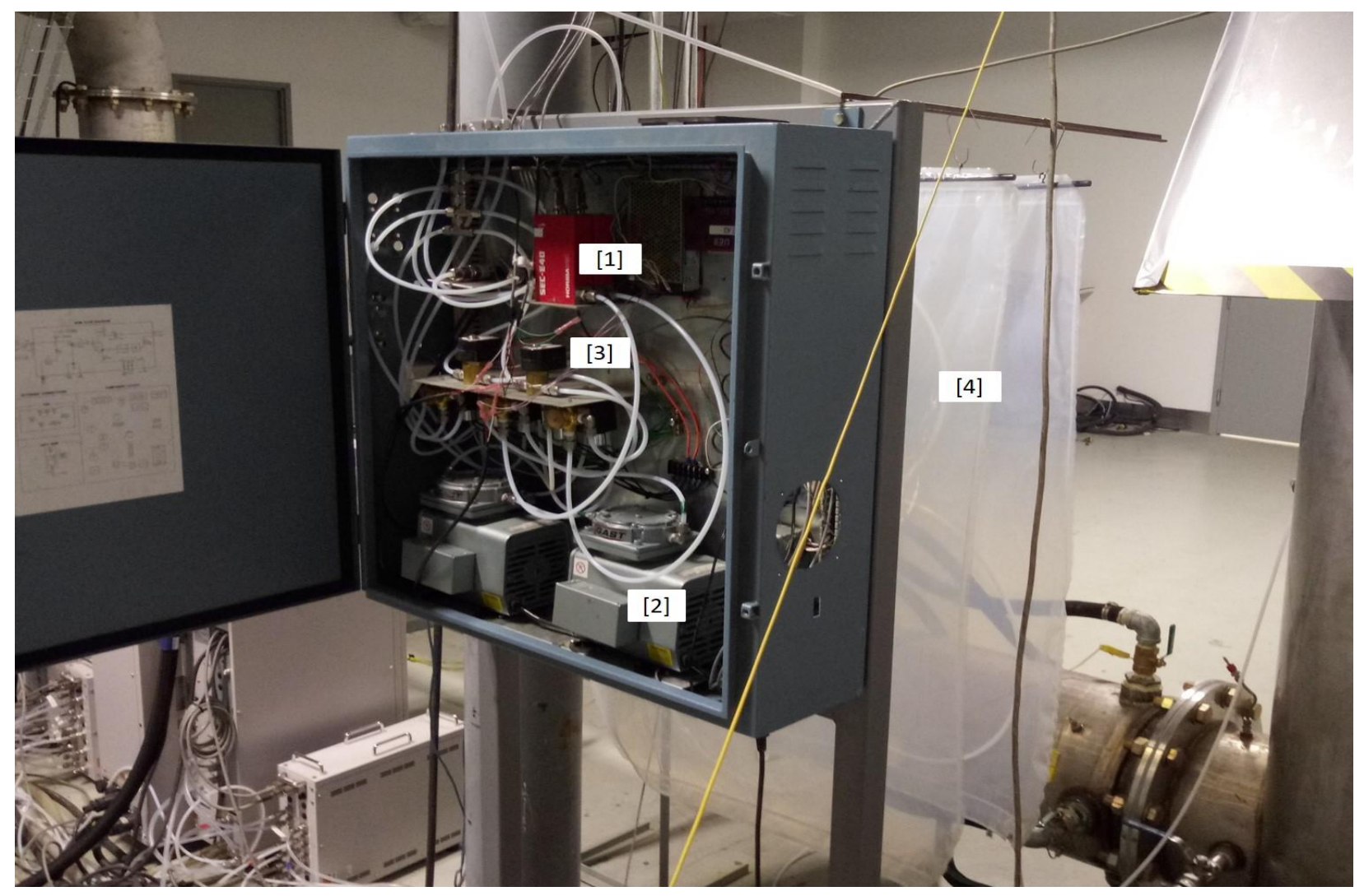

Figure 15 - Proportional Bag Sampling System: Mass flow controller [1], Vacuum pumps [2], Robust control of filling [3], Bagging System [4].

\subsection{Study Experimental Set-up}

Based on the scope of this work the following areas listed in sections below were considered and experiments were carried out to see their effects on the measurements of NOx at near zero 
level in a CVS set-up. As mentioned earlier this section further emphasize the specific section of the general experimental set-up used for conducting each of these studies.

Table 6- Summary of Experiments Carried Out

\begin{tabular}{|l|l|}
\hline Experiment & Section No \\
\hline Background Variability & 3.4 .1 \\
\hline Dilution air filtration study & 3.4 .1 .1 \\
\hline Proportional Bag Sampling & 3.4 .1 .2 \\
\hline Low Concentration NOx analyzer study & 3.4 .2 \\
\hline Virtual NO/NO2 injection Checks & 3.4 .3 \\
\hline Tunnel Heating & 3.4 .4 \\
\hline
\end{tabular}

\subsubsection{Background Variability Study}

The main purpose of this study is to account for variability in the ambient NOx and THC conditions as human operations, natural conditions amongst other activities tends to change the concentration of the constituents of the ambient air. The NOx and THC levels do change overtime and this variation will affect the accuracy of the measured NOx at this near zero level, this variation will be quite significant in using the CVS system to accurately quantify the emissions from exhaust which is already tending towards the near zero level. 


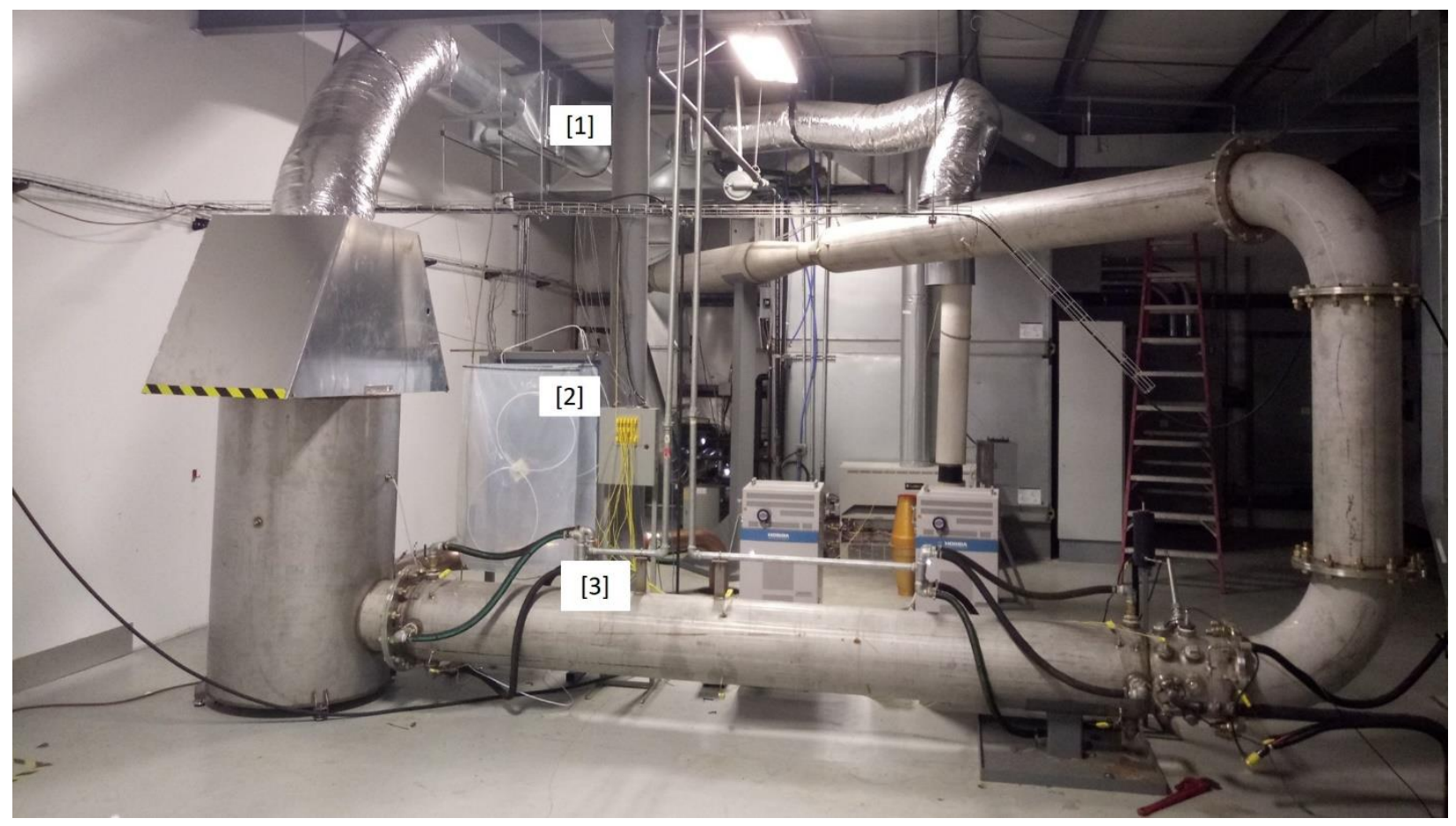

Figure 16- CVS Modifications Set-up: Dilution air filter [1], Proportional bag sampling [2], Tunnel heating set-up [3]

The ambient air collected via the air duct was sampled and the THC and NOx level were measured in this study. An hourly zero calibrate was done without spanning and calibrating to check the variability and stability of the NOx and THC measurements using analyzers. This study was carried out during the day, at night, when lab activities was in full operations and when it was not. This is to see the measure of the effects of human's activities and other conditions that can lead to variations in the background NOx and THC and as such can be accounted for and properly corrected in CVS measurement calculations.

\subsubsection{Dilution Air Filtration System}

This study was carried out to check the possibility of reducing variability in the measured background concentrations and to also maintain a consistent concentration all through sampling duration. This was carried out according to EPA “CFR, Title 40, Part 1065.140” standards for preconditioning of background air to reduce concentration and possibly remove constituents of the dilution air before it is mixed with the exhaust. This is to ensure testing are invariant of the changes in the background conditions or other factors that leads to contamination of the ambient air, which can either lead to an increase or a decrease in the amount of measured NOx or THC overtime. A dilution air filtration system was placed in the dilution air duct to filter the ambient air as shown 
in Figure 16 . Hence, reducing the NOx and THC levels and other air constituents that might have effect on accurately measuring the NOx at near zero level using the CVS measurement set-up.

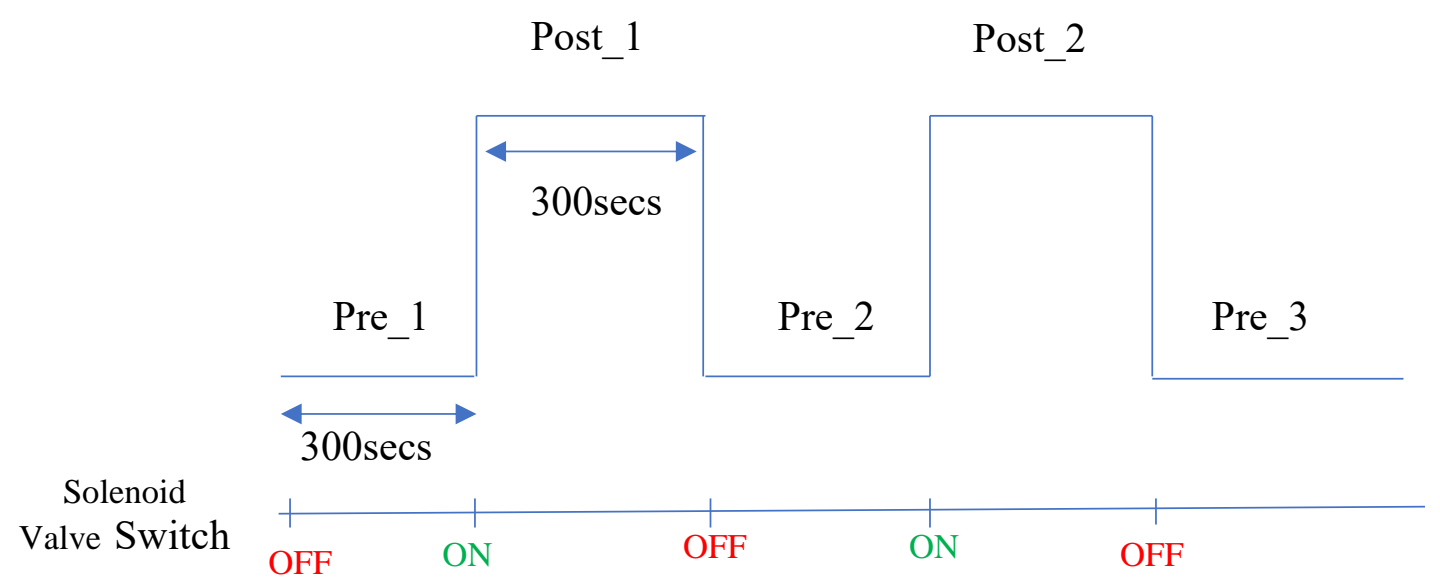

Figure 17 - Dilution air Filtration Pre and Post Sampling

This test was conducted similar to the background variability study with the integration of the activated charcoal filter in the set-up. A consecutive pre and post-sampling before and after the filter respectively was carried out for 300s along the air duct as shown in Figure 17. The intake air was also preconditioned by heating to investigate the effect of increase in temperature on the air filter reduction efficiency. This intermittent sampling was to account for possibly variability or measurement errors which might occur over long durations. This is to ensure an unbiased measurement was obtained for both pre and post sampling.

\subsubsection{Proportional Bag Sampling}

Proportional bag sampling (PBS) system was a study also carried out to account for the variability in background concentration which is not accounted for by assuming the background concentration is constant all the time. PBS was carried out according to EPA "CFR, Title 40, Part 1065.545" standards which ensured that proportional batch sampling using the bagging system was maintained all through the measurements. This study set-up as shown in Figure 15 ensures the filling of bagging system is not done at a constant flow rate as with the conventional method of bag sampling, rather the PBS system fills the dilute bag and the background bag proportional to the exhaust flow rate and dilution air flow rate respectively using the mass flow controller dedicated to each of the bagging sampling system. 
PBS was carried out at 4 different conditions which includes condition 1 when both dilute and background bag were both filled at constant flow rate and condition 2 when the background bag was filled at proportional flow rate while the dilute bag was filled at a constant flowrate. For condition 3 the background bag was filled at constant flow rate while the dilute bag filled was filled at proportional flowrate. While for condition 4 both bags were filled at proportional flow rate. The bag filling at the 4 different conditions were carried out during an FTP cycle and the respective emission measurements were quantified from the CVS set-up using a Horiba MEXAOne-CVS. The measurement obtained using the MEXA analyzer was used as a reference measurement to compare 3 other analyzers (FTIR, EcoPhysics and Brandgaus) which were used for quantifying the emissions from the bagging system after each respective FTP cycle. The bagging system used for the PBS were not heated.

\subsubsection{Low Concentration NOx Analyzer Study}

This study was conducted to see the suitability of the low NOx measuring devices in accurately measuring the low NOx concentration from the engine exhaust. Measurement error gotten from the measuring instrument due to limitations as a result of specifications is sure to affect the accurate quantification of NOx at this low level. Analyzer drifts and linearization accuracy of the analyzers were tests carried out in this study

\subsubsection{Analyzer Drift}

According to the EPA “CFR, Title 40, Part 1065.550” standards one of the required tests for gas analyzers used for emissions measurement is drift validation which incorporates the drift correction in its calculations. The analyzers drift for this study was carried concurrently as the background variability study. Zero and span readings for each analyzer were recorded every hour after the emissions from the bagging system had been evacuated and measured. This study was carried out multiple days to determine the hourly variation as a result of drift across each day. 


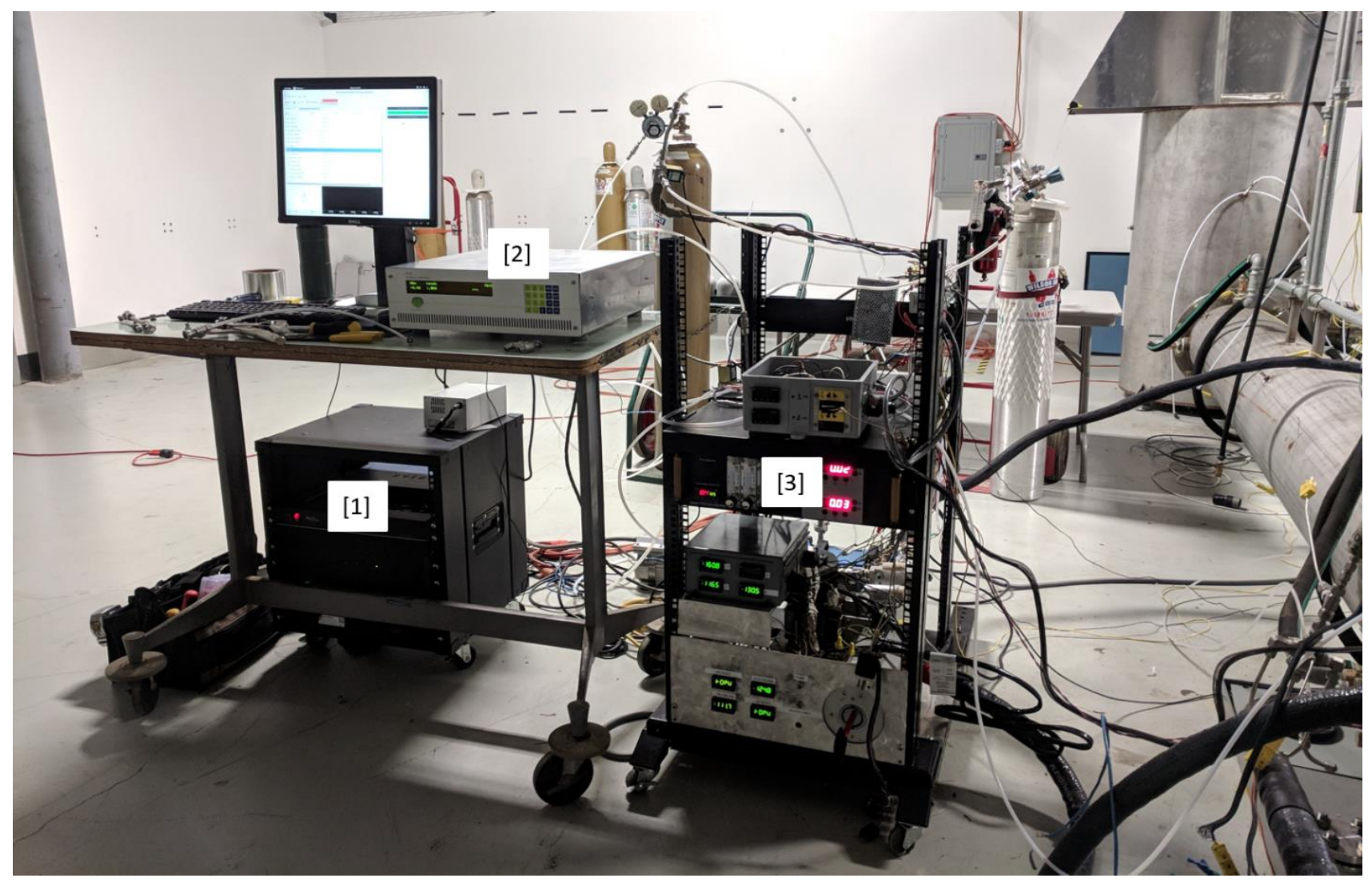

Figure 18 - Low concentration NOx analyzer set-up: DAQ system [1], Ecophysics CLD64 [2], Brandgaus 7707 [3].

\subsubsection{Analyzer Linearization}

Gas analyzers used in emission quantification are subjected to linearity verification according to the EPA "CFR, Title 40, Part 1065.307" standards. This ensure analyzers have accurate measurements within span of measurements. The low NOx analyzers linearization accuracy study was carried after zeroing and span-calibrating the analyzers. A calibrated gas divider according to EPA "CFR, Title 40, Part 1065.248" was used to increase the proportion of the calibration gas from $0-100 \%$ in intervals of $10 \%$ with $\mathrm{N}_{2}$ used as balance gas. $\mathrm{NO}_{2}, \mathrm{NOx}$ and compound of $\mathrm{NO}_{2}$ and NOx where used as calibration gases. In which $\mathrm{NO}_{2}$ and $\mathrm{NOx}$ calibration gases used $\mathrm{N}_{2}$ as balance gas while the for the compound calibration gas $\mathrm{NOx}$ was used as balance gas for $\mathrm{NO}_{2}$. The total NOx was measured by the Analyzers at each $10 \%$ increase for each calibration gas used respectively.

\subsubsection{Virtual NO/NO2 Injection Checks}

The verification of the entire CVS system and determination of the analyzer measurement consistency, using low NOx injection at low level was the essence of this experiment. Detection 
of leaks, NOx loss or hang-ups along specific sections of the CVS system can be easily inferred from this study. The injection was carried out in similitude to the propane check according to the EPA “CFR, Title 40, Part 1065.341” used to check CVS set-up integrity. For this study the NOx was injected into the dilute air stream at the tunnel entrance length using an injection kit which utilizes a mass flow controller (MFC). The MFC ensures a known quantity of $\mathrm{NO} / \mathrm{NO}_{2}$ reference gas is injected into the tunnel which is measured using the gas analyzers at sampling plane.

The measured quantity by the analyzers is a measure of the recovered mass of NOx. This study was carried out at 3 different sampling conditions which includes condition 1 when both the analyzer sampling and the tunnel were not heated. For condition 2 the analyzer sampling line was heated while the tunnel was not heated, while for sampling condition 3 both the analyzer sampling lines and the tunnel were heated. The condition 1 was carried out to see the effect of non-heated sampling lines as a result of faulty heaters along sampling line amongst other possibilities on the NOx measurement accuracy using the CVS sampling set-up at this low level.

\subsubsection{Tunnel Heating}

The heating of the CVS tunnel was recommended according to earlier works to eliminate water condensation in the exhaust dilution tunnel and sampling systems. According to the EPA "CFR, Title 40, Part 1065.140" standards for the dilution of gaseous emission constituents in a CVS tunnel, the dilution tunnel wall temperature should be close to that of the exhaust gas. The tunnel can either be insulated or heated to minimize or avoid water condensation which will aid in avoiding hang-up effects due to gas solubility caused from water-vapor condensation. Cold spots in the tunnel wall can also be avoided by limiting the temperature differences between wall surfaces while also ensuring all sampling surfaces are maintained well above the dew-point temperature of the exhaust gas. 


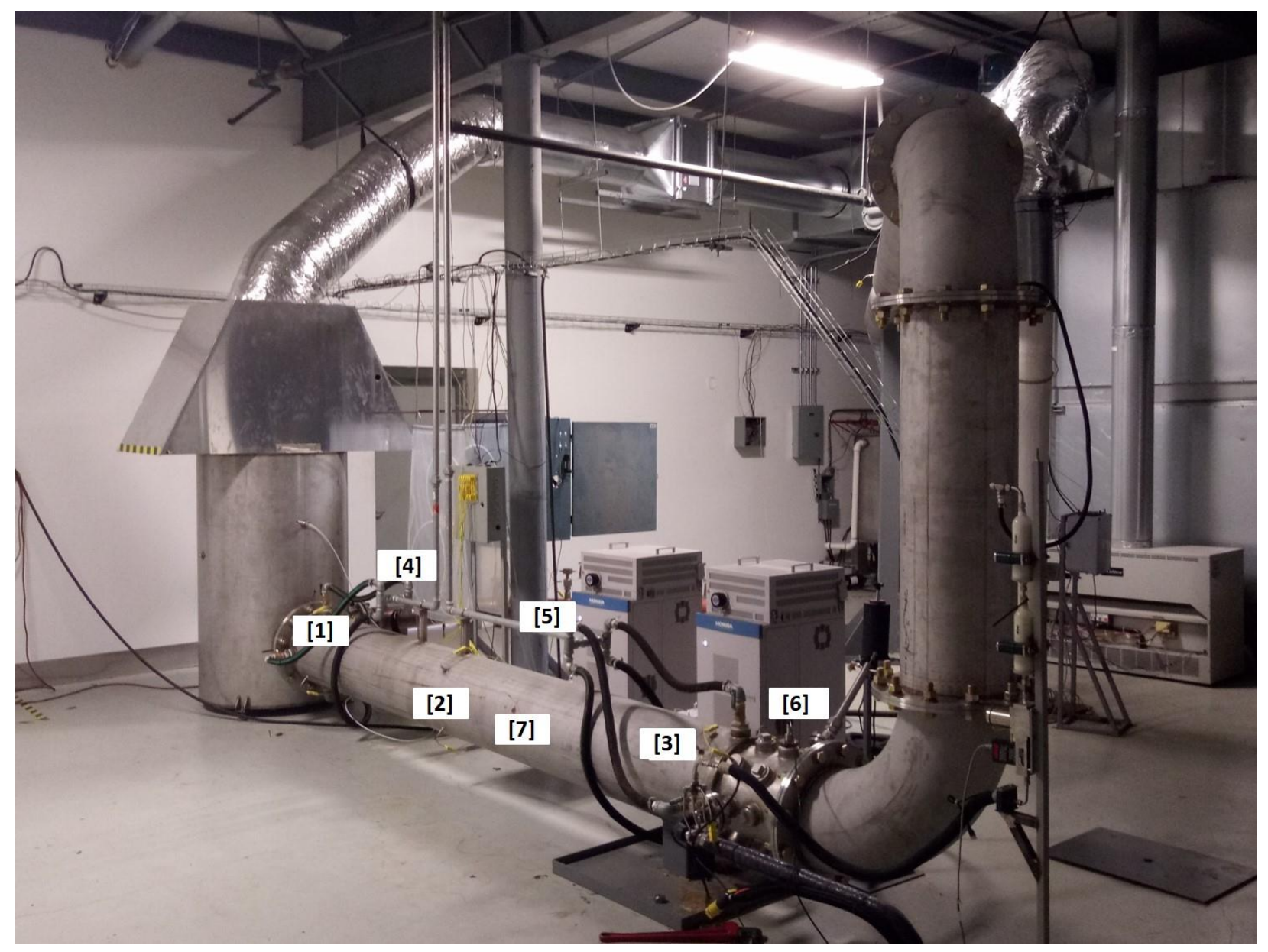

Figure 19- Tunnel Heating Set-up: Thermocouples locations [1,2,3], Dilution air and exhaust mixing chamber entrance [1], Hot water inlet [4], Water Outlet [5], Sampling Plane [6], Tunnel Heating Jacket [7].

This study checks the effect of tunnel heating of the CVS and the sample system as temperature and water content is a strong influential factor in accurately quantifying NOx at near zero level. 


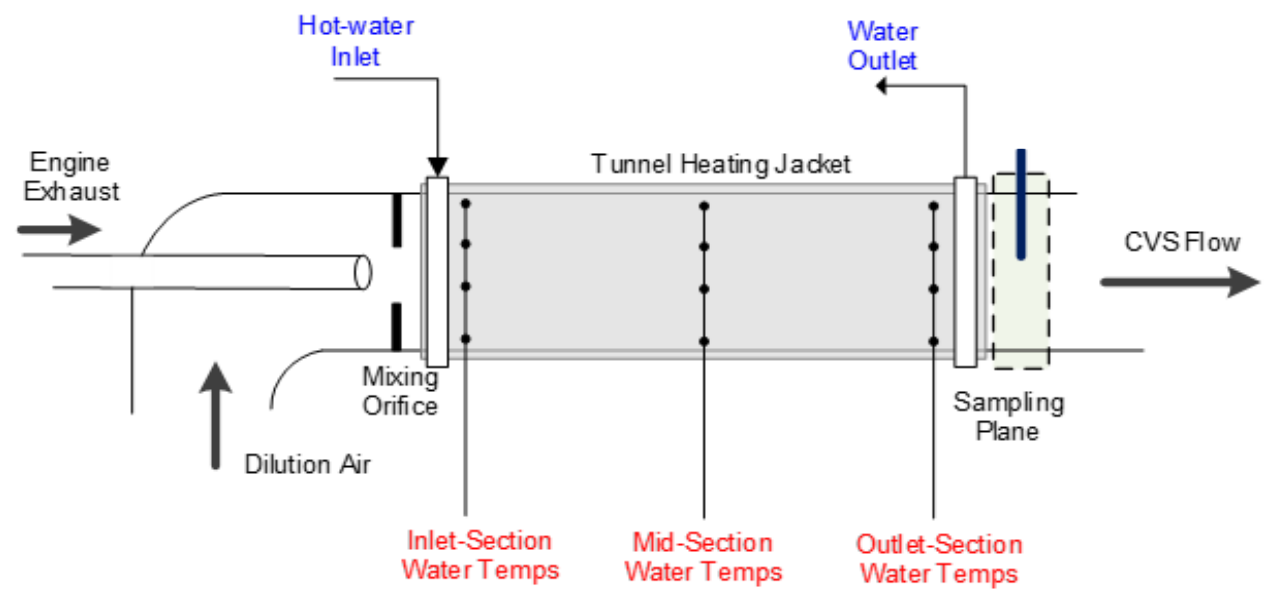

Figure 20- Schematic of the Tunnel Heating Set-up

The tunnel was heated by passing heated water system through the hot water inlet valve across the tunnel as shown in figure 20 and the water discharged via the water outlet valve. 12 thermocouples were integrated to monitor the tunnel jacket water temperature which were used as a feedback mechanism to control the inlet water valves while keeping the outlet water valves wide open. The inlet water jacket valves were adjusted accordingly to optimize the tunnel heating thereby ensuring stable water temperature along the thermocouples which will aid minimal temperature differences between wall surfaces along the CVS tunnel. This aid in minimizing possibility of cold spots along the tunnel while keeping the temperature of the tunnel wall surfaces above the dew point of the diluted exhaust gas. 


\section{Results and Analysis}

This section of the report gives an overview of the results gotten for each study and the analysis carried out in achieving the objectives of this study.

\subsection{Background Variability Study}

\subsubsection{NOx Variability}

This study was carried out to determine daily variations in ambient NOx concentrations across each day. Brandgaus and Ecophysics low NOx analyzers were used, and the data gotten from the experiment for each day was binned into 10 minutes to see the variation in NOx concentration within 600seconds interval.
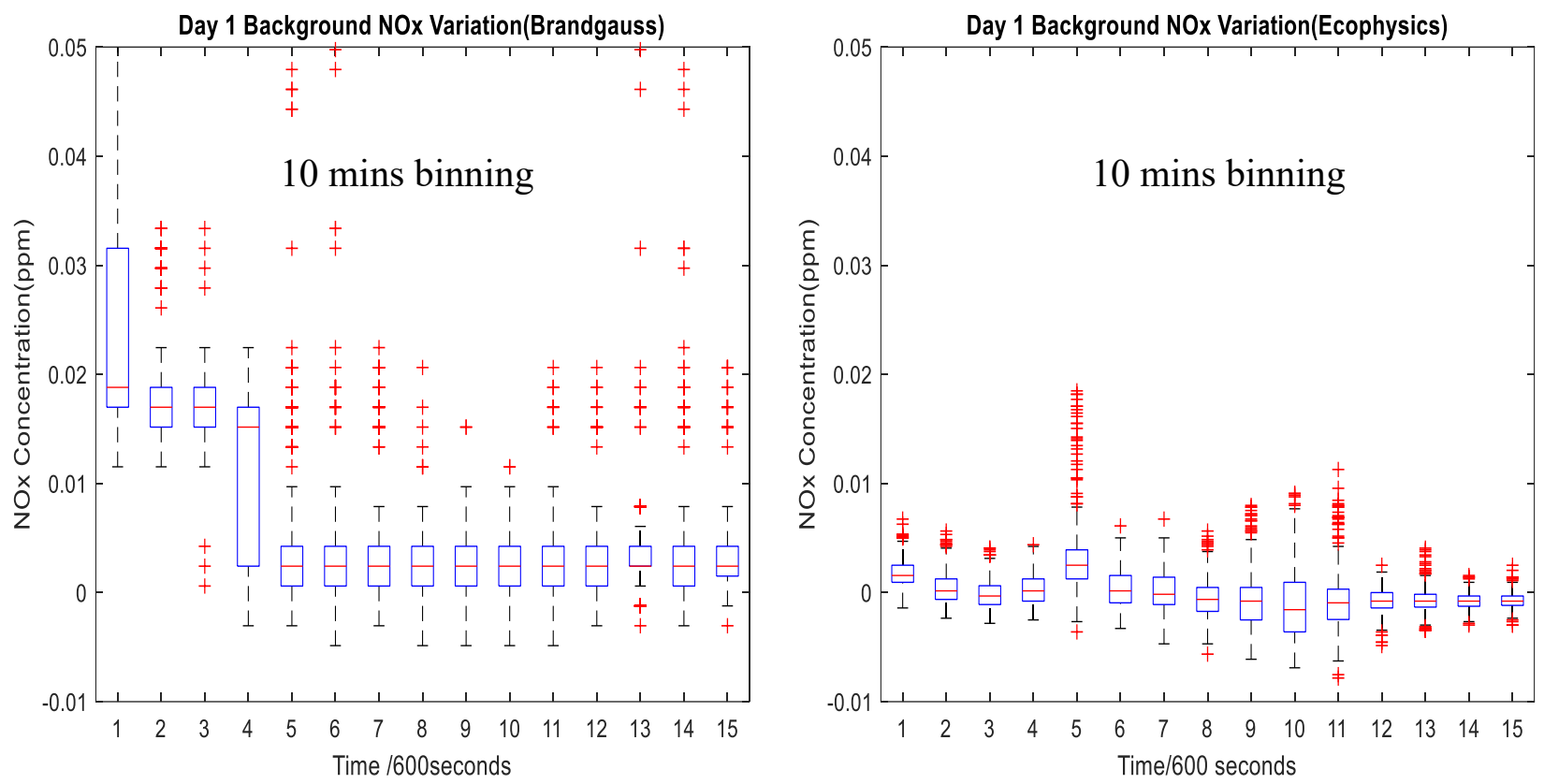

Figure 21- Variation of Background NOx Concentration across Day 1

There was observed variation in the amount of background NOx measured by both low analyzers within 10 minutes interval as shown in Figure 21. The reason for this variation might have been due to the differences in each analyzer capabilities as a result of their specifications. This might have also resulted from other possible random errors relating to sampling connections and measurements. The measurements were in the range of $0-0.05 \mathrm{ppm}$ for the Brandgauss analyzer while for the eco-physics analyzer lesser variations within $0.01 \mathrm{ppm}$ range was measured. Daily variation of NOx concentration was also noticed for both analyzers on day 2 which is depicted in 
figure 20 for both trace level analyzers. This connotes variability in the amount of NOx in the dilution air used for the CVS emission measurement system.
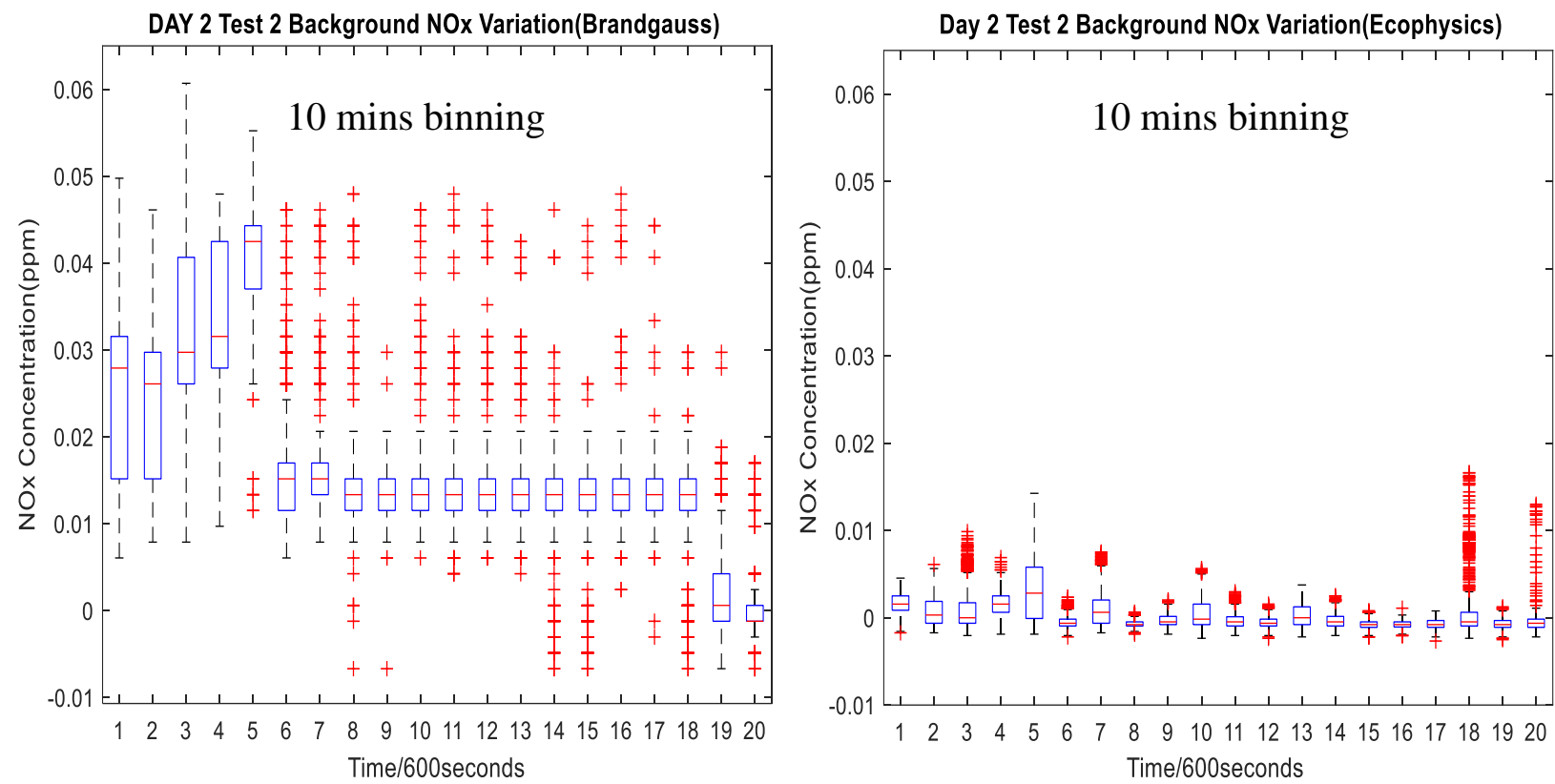

Figure 22- Variation of Background NOx Concentration across Day 2(Test 2)

Higher NOx concentrations was measured for each analyzer on day 2 compared to day 1 (test 1 day 2 attached in appendix) as shown in test 2 for day 2 in Figure 22. The rationale is to depict how much variation can be obtained in measurement as a result of analyzers difference and specifications. The maximum NOx measurement for the second day was approximately $0.06 \mathrm{ppm}$ for the Brandgaus analyzer and $0.014 \mathrm{ppm}$ for the Ecophysics analyzer. This represents a $20 \%$ and $40 \%$ increase in concentrations respectively for both Brandgaus and Ecophysics analyzers compared to day 1. The average NOx concentration measured for the Eco-physics analyzer is $0.00088 \mathrm{ppm}$ and $0.0077 \mathrm{ppm}$ for day1 and day 2 respectively. While the Brandgaus analyzer had an average of $0.0069 \mathrm{ppm}$ for day 1 and $0.017 \mathrm{ppm}$ for day 2 .

Overtime variation in NOx concentration within 10 minutes interval on a daily basis, and variations associated with day to day activities is certain to affect being able to quantify NOx at near zero levels accurately. This results from the fact that as the difference between the background NOx concentration and the diluted exhaust NOx concentration gets smaller it becomes more difficult for the analyzer to quantify NOx. There is also the possibility of the NOx concentration of the dilution air getting higher than that of the diluted exhaust as a result of variation, and this poses more challenge in quantifying NOx using the CVS system set-up at this low level 


\subsubsection{THC Variability}

Daily variation of total hydrocarbon constituents in background air was simultaneously studied alongside the NOx variability study. MEXA analyzer was used for the THC measurement variation comparison across days as shown in figure 21 . THC variation for day one ranged from $2.3 \mathrm{ppm}$ to approximately $2.9 \mathrm{ppm}$, while for the second day the THC variation was higher with a range of $2.4 \mathrm{ppm}$ to approximately $3.2 \mathrm{ppm}$. This represents a $133.33 \%$ increase in range across both days.
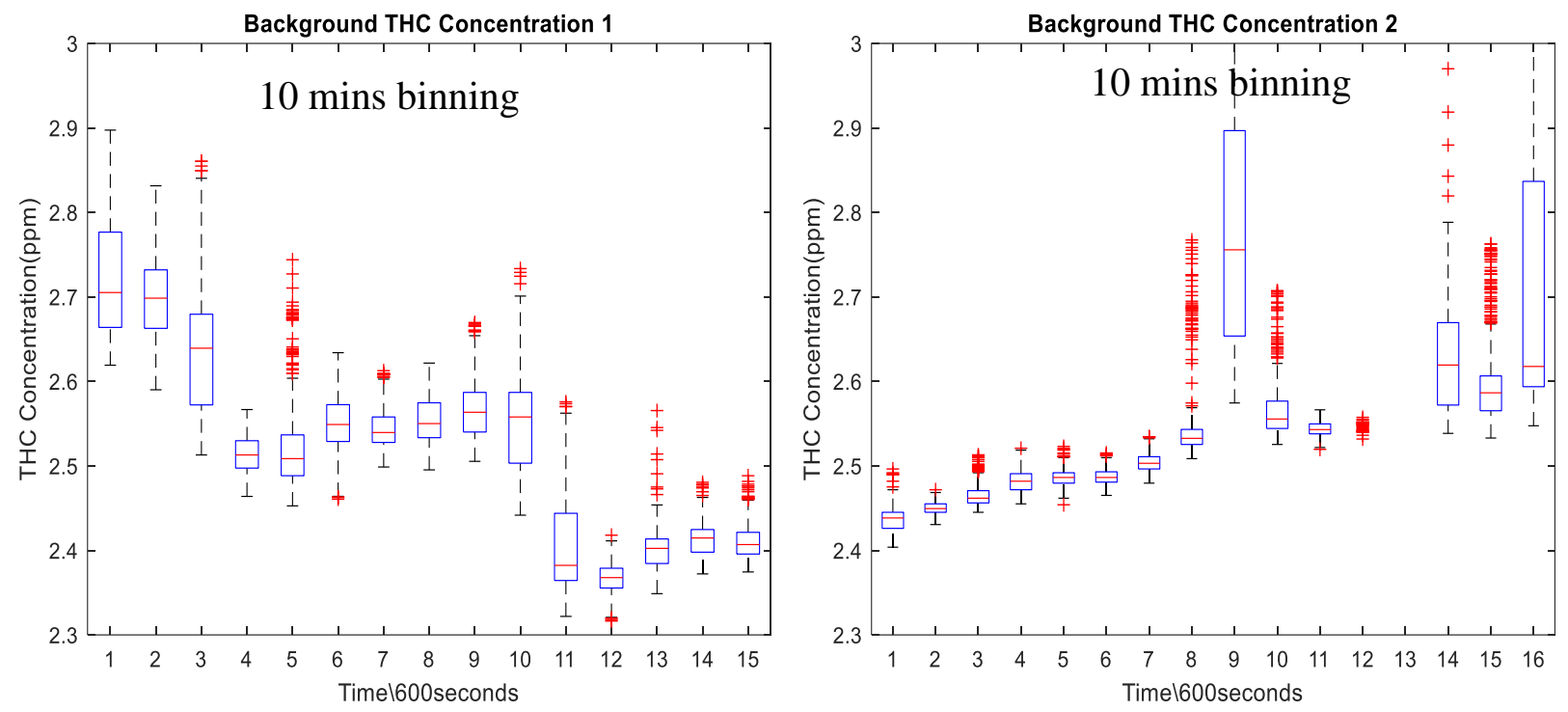

Figure 23-Background THC Concentration: Day 1 and Day 2

The average THC for day 1 and day 2 were $2.53 \mathrm{ppm}$ and $2.2 \mathrm{ppm}$ respetively. Which connotes a $13 \%$ decrease in average THC across the 2 days. There was observed decrease in THC concentration for day 1 while there was observed increase in THC concentration for day 2 as depicted in Figure 23.This variation in THC concentration across days is certain to affect the accurate measurment of NOx at low level .As THC contanination results in quenching which affects the accuracy of the CLD measurement technique used in NOx measurement.

\subsubsection{Dilution Air Filtration System}

After investigating the variability in background NOx and THC and there was evidence of variation in the concentration of both species. A dilution air filter was integrated into the dilution air system to minimize or possible mitigate this variation across sampling period. Hence, sampling was carried out before (PRE) and after (POST) the dilution air filtration system to ascertain the 
effectiveness of this modification of the CVS set-up. The reason for this enhancement of the CVS system is to reduce variability in ambient NOx and THC concentration during sampling period thereby ensuring stable specie concentrations all through . The test was replicated under different conditions as shown in Table 7 and the values of the NOx and THC was sampled using Brandgauss and Eco-physics low NOx analyzers which measured NOx concentrations only, while the MEXA measured THC concentrations.

Table 7 -Dilution Air Filter Average NOx and THC Reduction with Different Testing Conditions

\begin{tabular}{|l|l|l|l|l|}
\hline Conditions & Test 1 & Test 2 & Test 3 & Test 4 \\
\hline Avg. Intake Temp $\left[{ }^{\circ} \mathrm{C}\right]$ & 26 & 21 & 39 & 35 \\
\hline Avg. Intake Humidity $[\%]$ & 75 & 84 & 26 & 36 \\
\hline
\end{tabular}

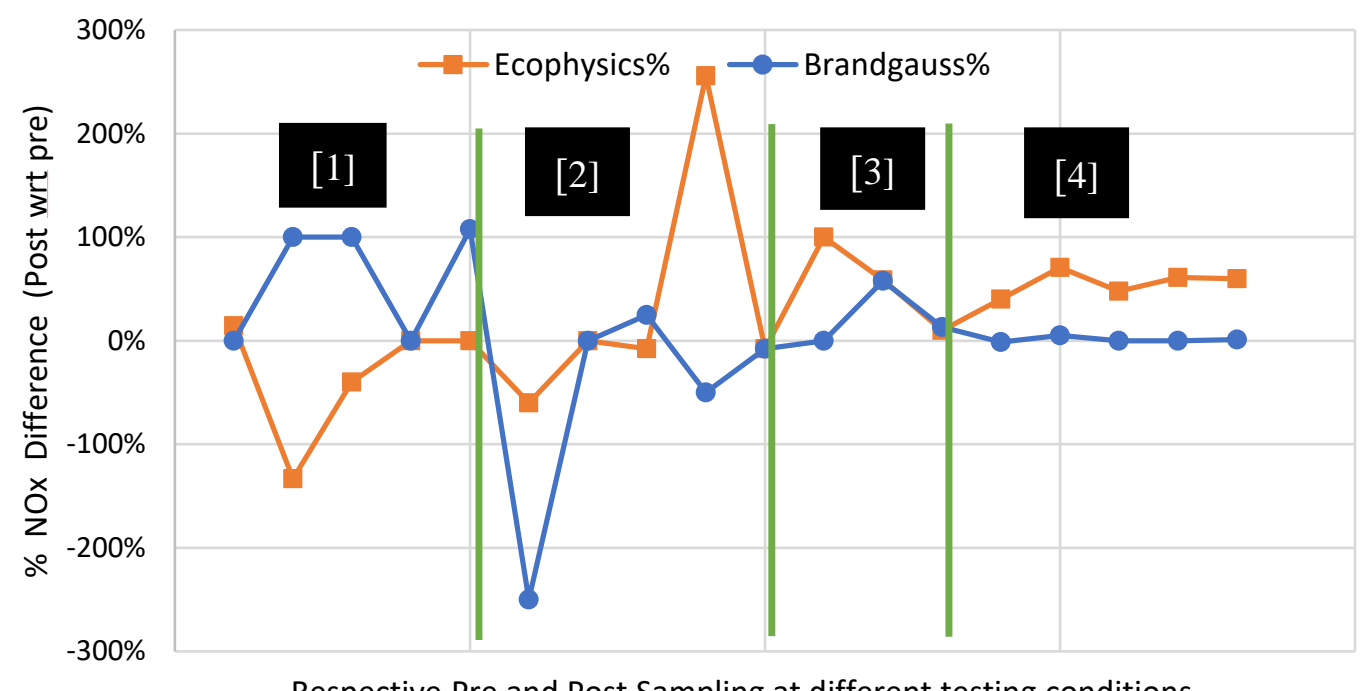

Respective Pre and Post Sampling at different testing conditions

Figure 24- Dilution Air Filter \% Reduction in ambient NOx : Test 1 [1], Test 2 [2],Test 3 [3], Test 4 [4] 
Table 8- Summary of Average NOx Reduction Across Testing Conditions

\begin{tabular}{|l|l|l|l|l|l|}
\hline Analyzers & \multicolumn{1}{|c|}{ Conditions } & \multicolumn{1}{c|}{ Test 1 } & \multicolumn{1}{c|}{ Test 2 } & Test 3 & \multicolumn{1}{c|}{ Test 4 } \\
\hline \multirow{3}{*}{ Ecophysics } & Avg Pre(ppm) & 0.0006 & -0.0004 & 0.0095 & 0.011 \\
\cline { 2 - 6 } & Avg Post(ppm) & 0.0004 & -0.00008 & 0.0051 & 0.0062 \\
\cline { 2 - 7 } & Avg \%NOx Reduction & $22 \%$ & $76.7 \%$ & $45.8 \%$ & $42.8 \%$ \\
\hline \multirow{3}{*}{ Brandgauss } & Avg Pre(ppm) & -0.0014 & -0.004 & 0.037 & 0.079 \\
\cline { 2 - 7 } & Avg Post(ppm) & -0.0006 & -0.005 & 0.029 & 0.077 \\
\cline { 2 - 7 } & Avg \%NOx Reduction & $53.8 \%$ & $-19.3 \%$ & $21.2 \%$ & $1.5 \%$ \\
\hline
\end{tabular}

Percent NOx reduction as a result of incorporating the dilution air filter to the CVS measurement set-up was as much as $250 \%$ and $100 \%$ as measured by both Eco-physics and Brand-gauss analyzers respectively. There was also percent increase in measured NOx between pre and post sampling as measured by both analyzers as shown in Figure 24 . This occurrence can be attributed to the pre and post sampling being carried out consecutively rather than concurrently. From the background variability study, it was evident that variation in background NOx occurs within short time frame. Hence, this might have been the cause of the increase in measured NOx recorded by both analyzers, since the NOx concentration might have increased during post-sampling. The average percent difference in measured NOx between the pre and post was $47 \%$ and $14.3 \%$ for the Eco-physics and Brand-gauss analyzers respectively.
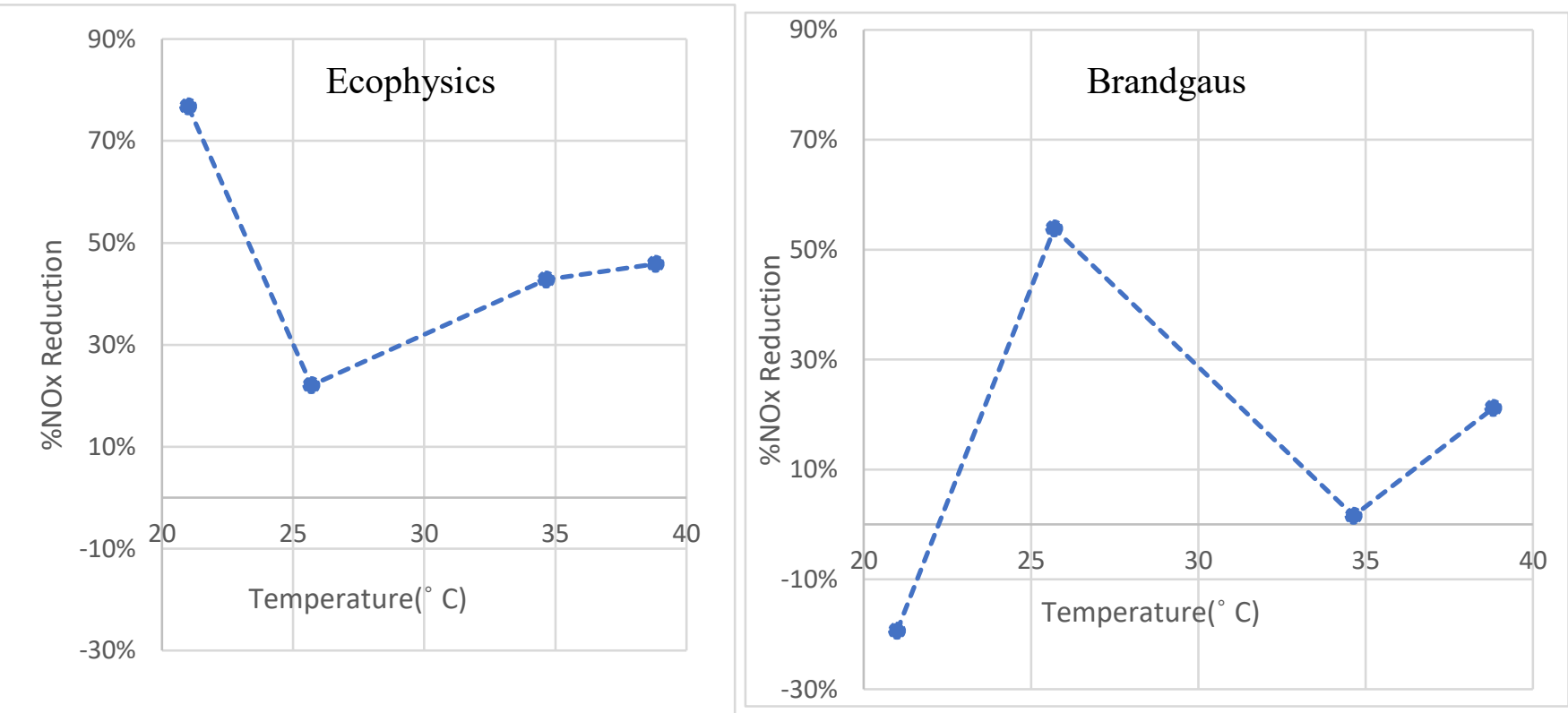

Figure 25- Effect of Increase in Temperature on \% NOx Reduction 
The effect of increase in temperature on NOx reduction as measured by both Ecophysics and Brandgaus analyzers does not showcase a definite linear relationship as shown in Figure 25.
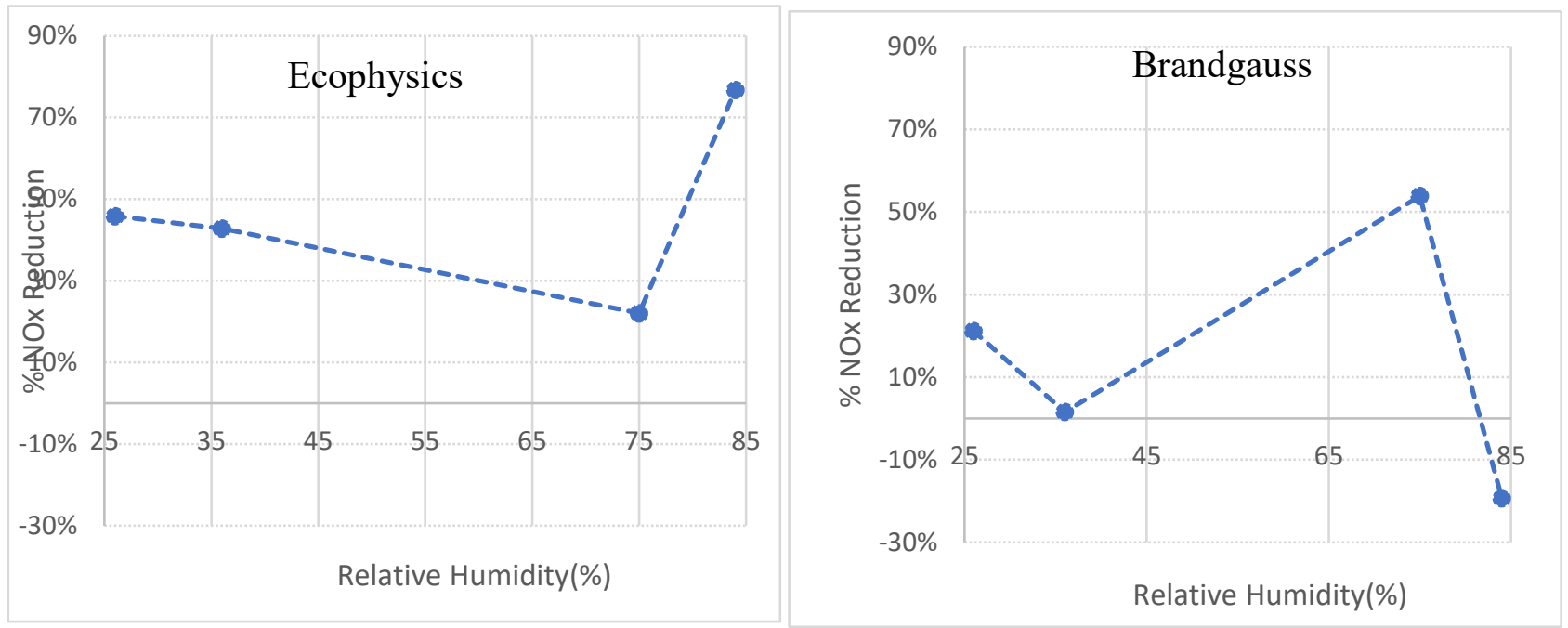

Figure 26- Effect of Increase in Relative Humidity on \% NOx reduction

Also, the effect of increase in humidity does typically showcase a specific trend on reducing NOx concentration in the ambient air as shown in Figure 26.

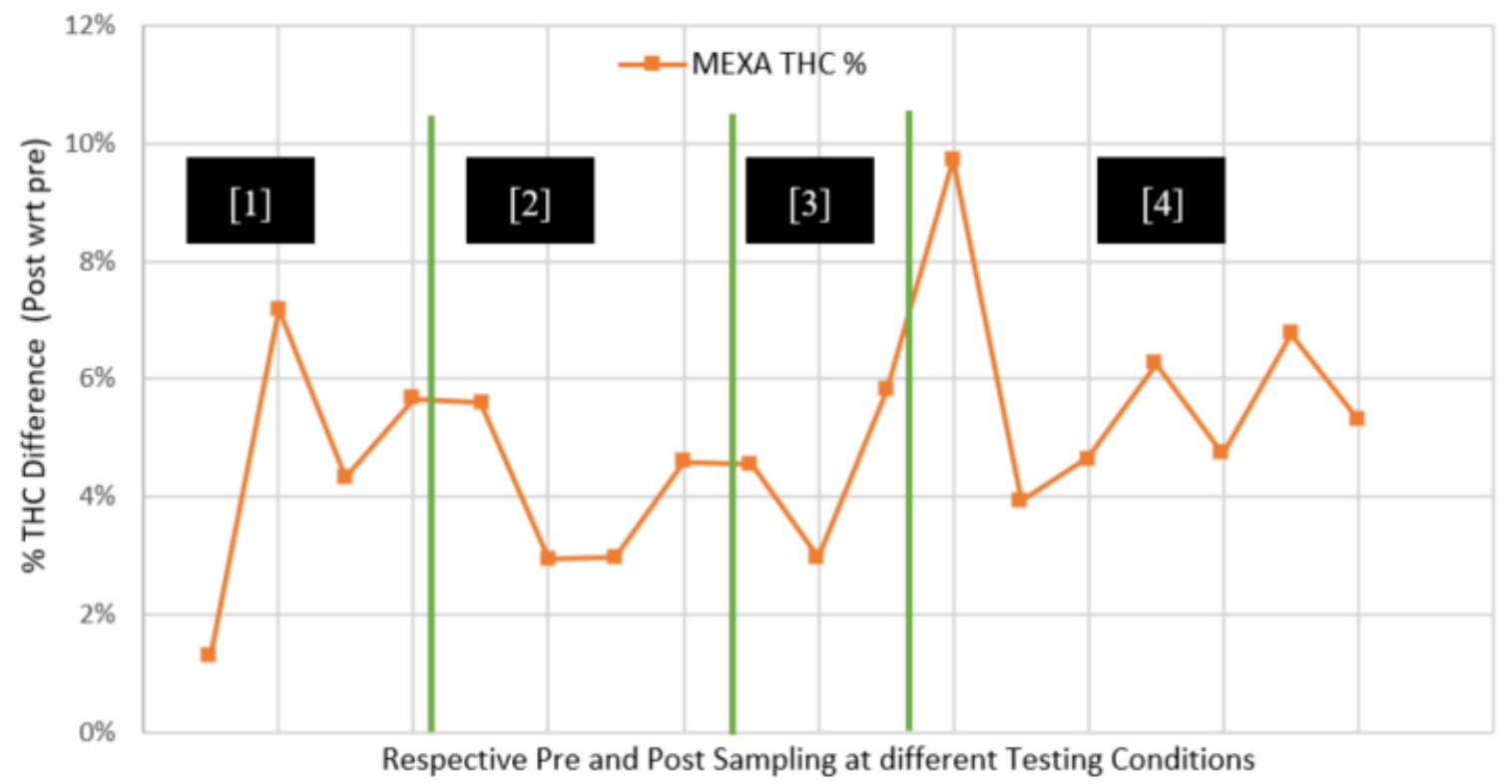

Figure 27 - Dilution Air Filter \% Reduction in ambient THC: Test 1 [1],Test 2 [2],Test $3[3], \quad$ Test 4 [4] 
Table 9- Summary of Average THC Reduction Across Testing Conditions

\begin{tabular}{|c|l|l|l|l|l|}
\hline Analyzer & \multicolumn{1}{|c|}{ Conditions } & Test 1 & \multicolumn{1}{c|}{ Test 2 } & \multicolumn{1}{c|}{ Test 3 } & \multicolumn{1}{c|}{ Test 4 } \\
\hline \multirow{3}{*}{ MEXA } & Avg Pre $(\mathrm{ppm})$ & 3.04 & 3.05 & 3.30 & 3.21 \\
\cline { 2 - 6 } & Avg Post $(\mathrm{ppm})$ & 2.89 & 2.94 & 3.10 & 3.03 \\
\cline { 2 - 6 } & Avg \% THC Reduction & $4.8 \%$ & $3.6 \%$ & $6.5 \%$ & $5.5 \%$ \\
\hline
\end{tabular}

From THC percent reduction was maximum of $10 \%$ as measured by the MEXA analyzer. It can be inferred that the DAR was more effective in reducing background THC in test 1 compared to test 2 conditions. The DAR was most effective at Test 4 with a $10 \%$ reduction in ambient THC concentration. The intermittent switching between high and low THC reduction might possibly be due to variation in ambient air. For this study the average percentage difference between pre and post sampling of THC is 5\%. At near zero levels this reduction in ambient THC concentrations are significant in aiding accurate NOx measurement as background THC variability is reduced as a result.

The dilution air filter showcases an increase in \% THC reduction with increasing temperature and a decrease in \% THC reduction with increasing relative humidity as shown in Figure 28. It can be inferred that high temperature and low humidity will be an optimum condition for the dilution air filter to effectively and efficiently reduce THC.
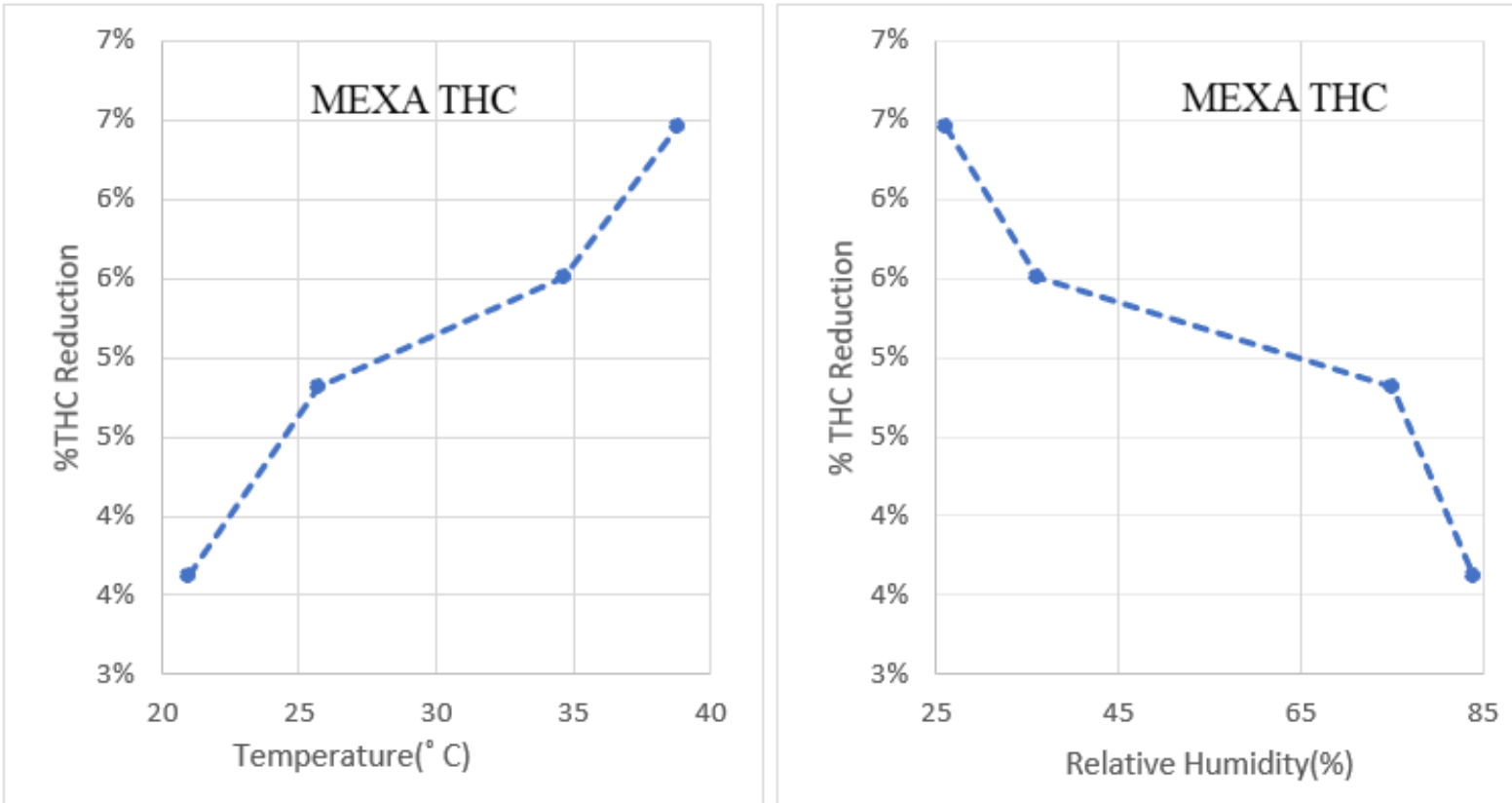

Figure 28 - Effect of increasing Temperature and Relative Humidity on \% THC Reduction 


\subsubsection{Proportional Bag Sampling}

Proportional bag sampling was carried out to factor in variations observed during measurement, to ensure the average bag measurement is representative of the actual emissions. Emission measurement using the bagging system sampling was carried out using 4 different modes as shown in table 8 to see the effect of proportional sampling on the measured values of NOx in grams using the CVS set-up. The error difference in the amount of NOx in grams measured using the CVS system was compared to the bagging system. There was observed increased with changing from constant sampling mode to proportional sampling mode for both the dilute and background bag sampling.

Table 10 - Modes of Bag Sampling

\begin{tabular}{|l|l|l|l|l|}
\hline Sampling Bag & Mode 1 & Mode 2 & Mode 3 & Mode 4 \\
\hline Background & Constant & Proportional & Constant & Proportional \\
\hline Dilute & Constant & Constant & Proportional & Proportional \\
\hline
\end{tabular}

The error difference measured by the ECO-physics analyzers was approximately within $30 \%$ on average for mode 1 and mode 2 and around $50 \%$ on average for mode 3 with the largest error difference of approximately $80 \%$ which was observed at mode 4 when both bags were filled proportionally as shown in Figure 29. The Brandgaus and the FTIR analyzers both had approximately $30 \%$ error difference in NOx measured in grams for the first 2 modes. The 3rd mode had an average difference of $50 \%$ for both analyzers. The largest error difference was observed at mode 4 which was approximately $90 \%$ for both Brandgaus and FTIR analyzers similar to that of the eco-physics analyzer.

From the results gotten it is evident that there is variation as a result of proportional bag sampling compared to constant bag sampling. From earlier works proportional bag sampling offers more accuracy than constant bag sampling since it accounts for variation in exhaust flow rate and concentration changes during testing. Variation in the measurement obtained from constant bag sampling and proportional bag sampling of NOx, compared to the CVS measurements was observed in Figure 29. 


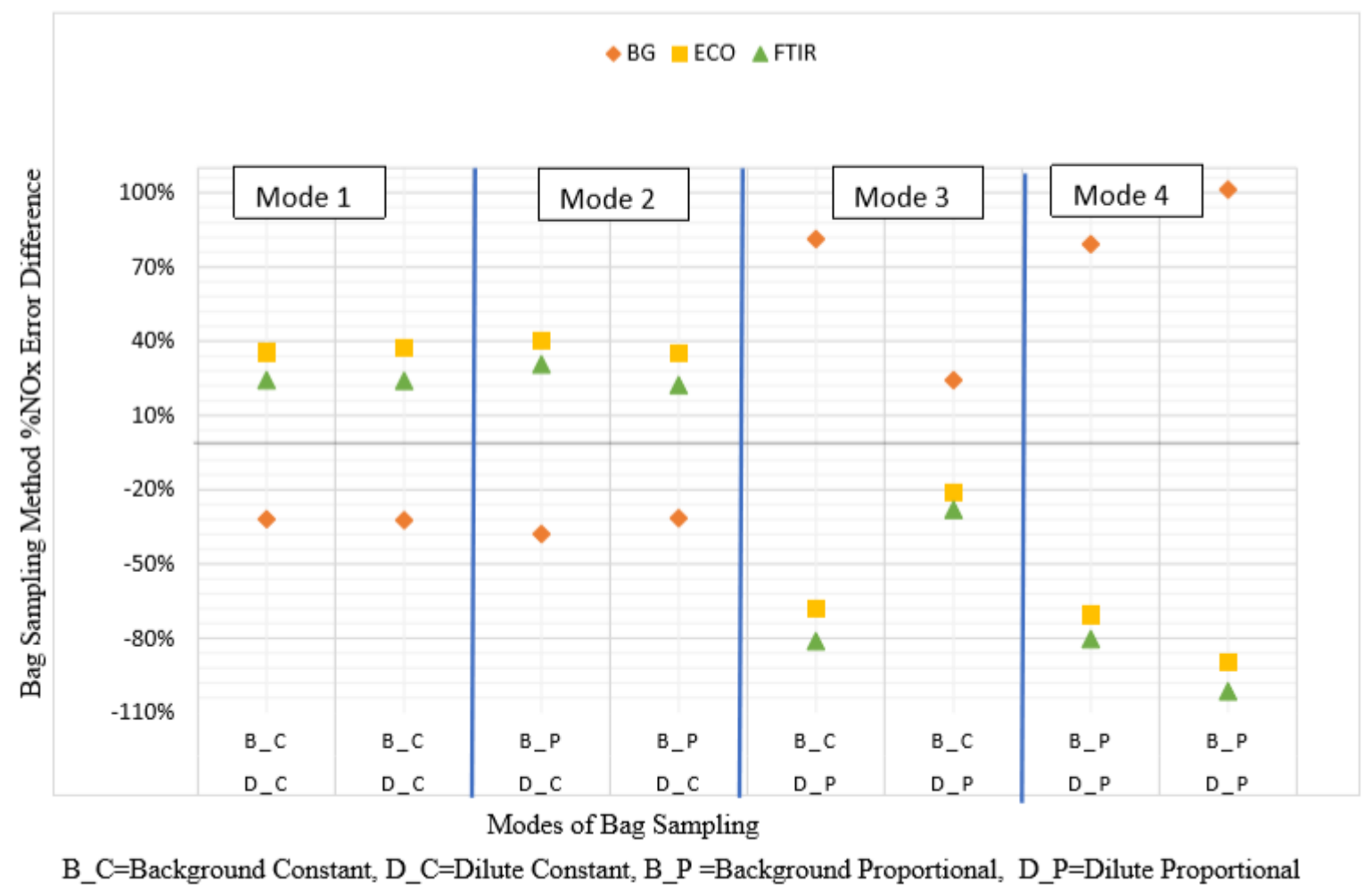

Figure 29- Percent Error Difference in NOx Measured to CVS Measurement

Analysis of variance of the measurements gotten from using the CVS and the proportional bag sampling measuring systems showcase that the CVS measurements are independent of the sampling modes as shown in Figure 30 with a P-value of 0.2441 which is significant at the 0.05 level. This connotes the CVS mean measurements are the same at the 4 sampling modes. For the PBS measurements obtained from the FTIR there exist great difference between the means of the sampling modes at 0.05 significant level as shown in figure 30 . This is also evident in the studentsT test comparison of the sampling modes carried out as shown in Figure 30.

This connotes that a more accurate bag sampling measurement can be achieved with proportional bag sampling compared to the constant bag sampling. As this will aid in better quantification of measured NOx values using a CVS system set-up at near zero level. Though there is possibility of 
the error between the CVS and the PBS measurements coming as a result of water condensation in the bagging system since the bagging system used in this study was not heated.

CVS

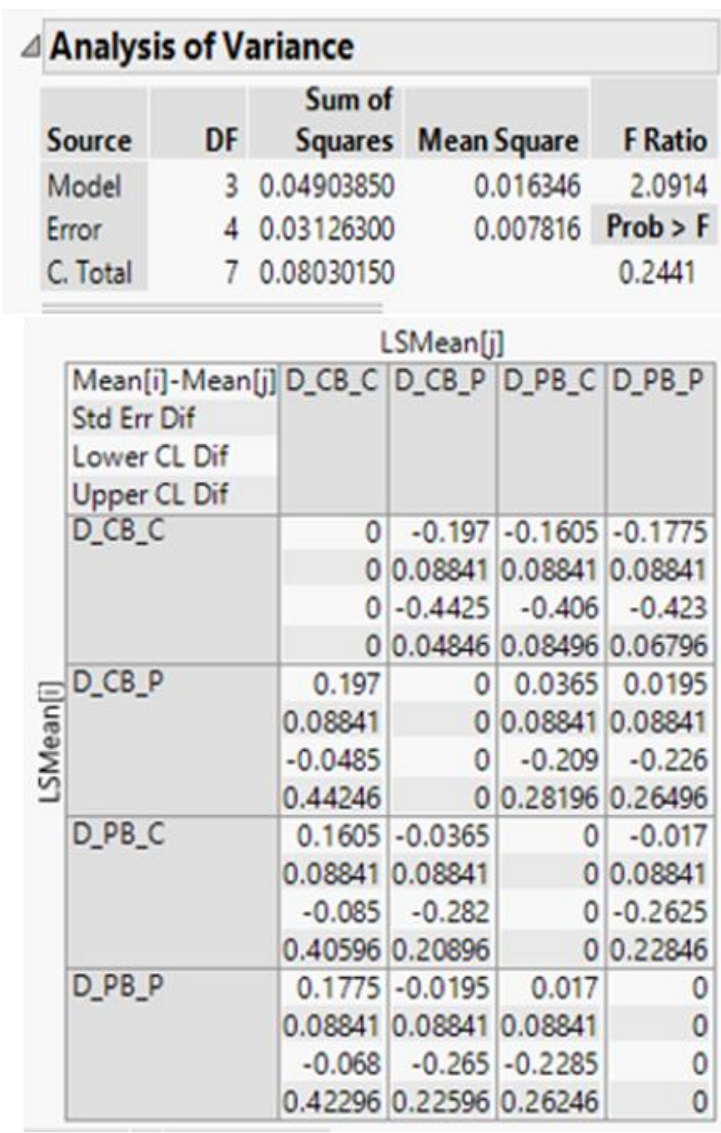

PBS(FTIR)

\begin{tabular}{|l|r|r|r|r|}
\hline \multicolumn{4}{|l|}{ Analysis of Variance } & \\
\hline Source & DF & $\begin{array}{r}\text { Sum of } \\
\text { Squares }\end{array}$ & Mean Square & F Ratio \\
\hline Model & 3 & 0.37632650 & 0.125442 & 13.1536 \\
\hline Error & 4 & 0.03814700 & 0.009537 & Prob > F \\
C. Total & 7 & 0.41447350 & & $0.0154^{*}$ \\
\hline
\end{tabular}

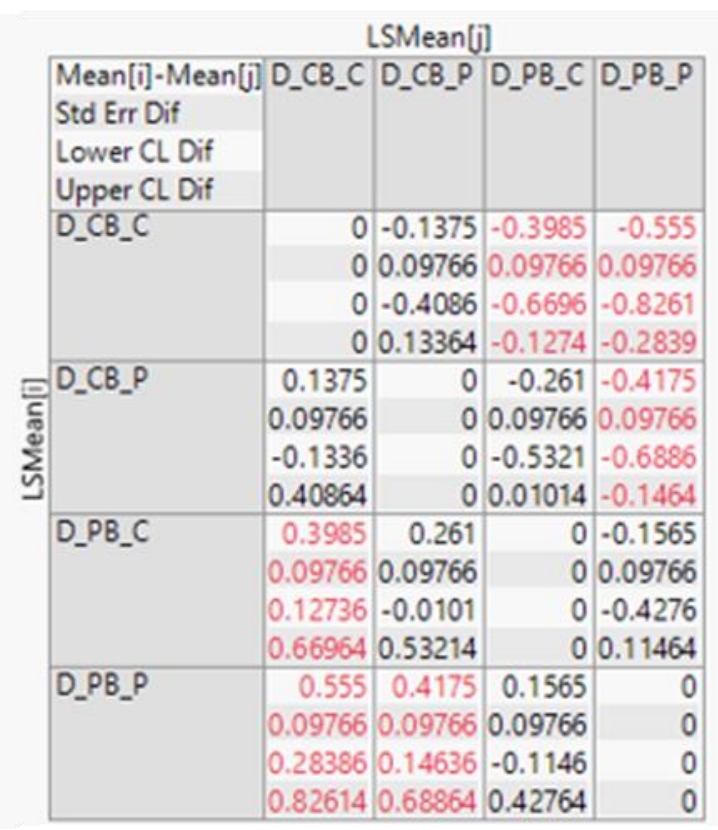

Figure 30- ANOVA and Student-T Comparison for CVS and FTIR NOx Measurement at Different Sampling Modes

\subsection{Low Concentration NOx Analyzers Comparison}

Low concentration NOx analyzers which were used for this study were compared based on their measuring parameters such as drift and linearization accuracy. The accuracy of each analyzers to accurately measure NOx compounds was also considered in this study.

\subsubsection{Analyzer Drift}

The low NOx analyzers had overtime drift when measuring a standard calibration gas. The percent drift decreased, increased and fluctuated for both low NOx analyzers. The Ecophysics was more stable and had a minimal hourly zero drift compared to the Brandgaus hourly zero drift. Both analyzers showcased higher span drift error as shown in Figure 33 and 34. 


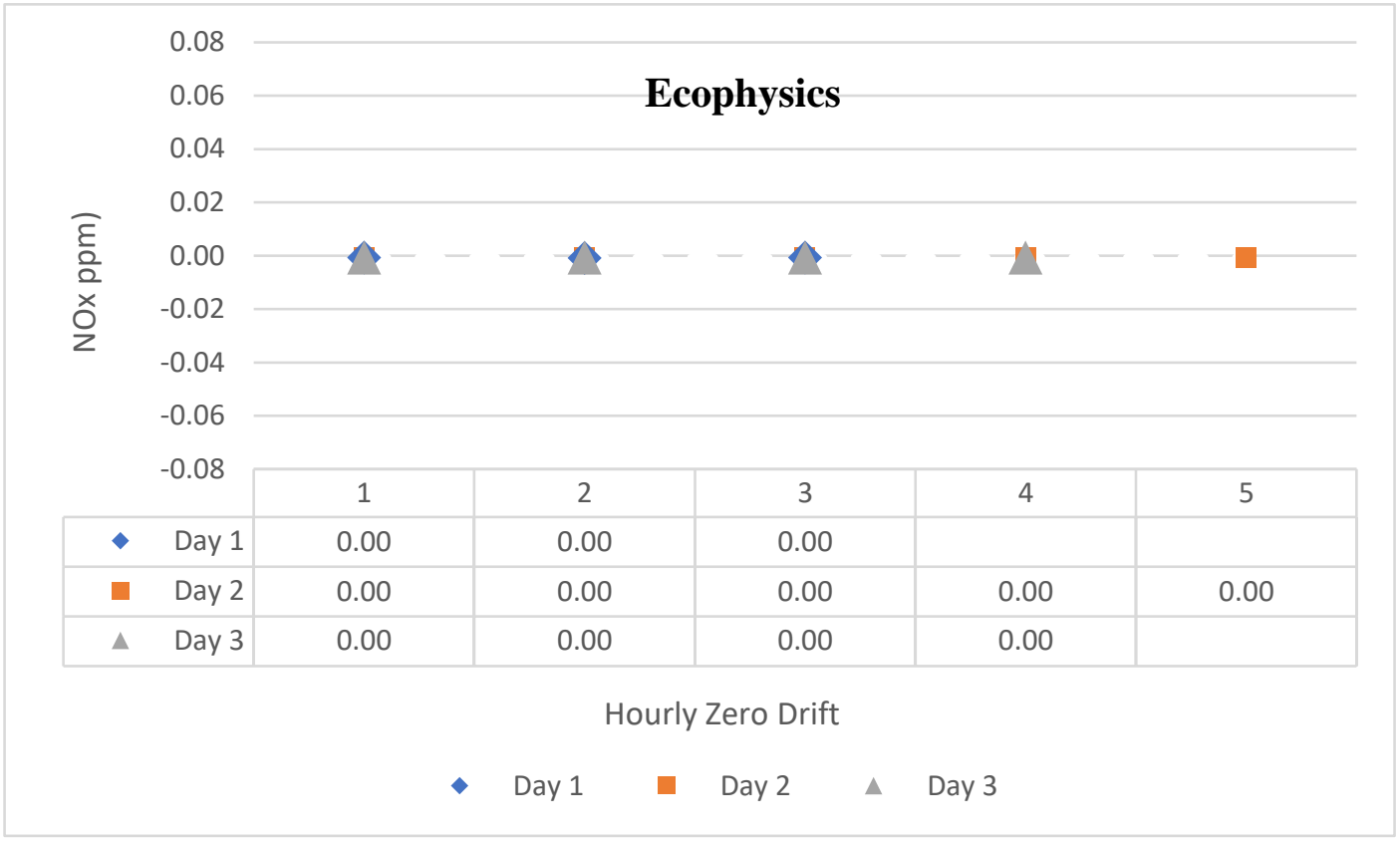

Figure 31- Ecophysics NOx Analyzer Daily Hourly Zero Drift

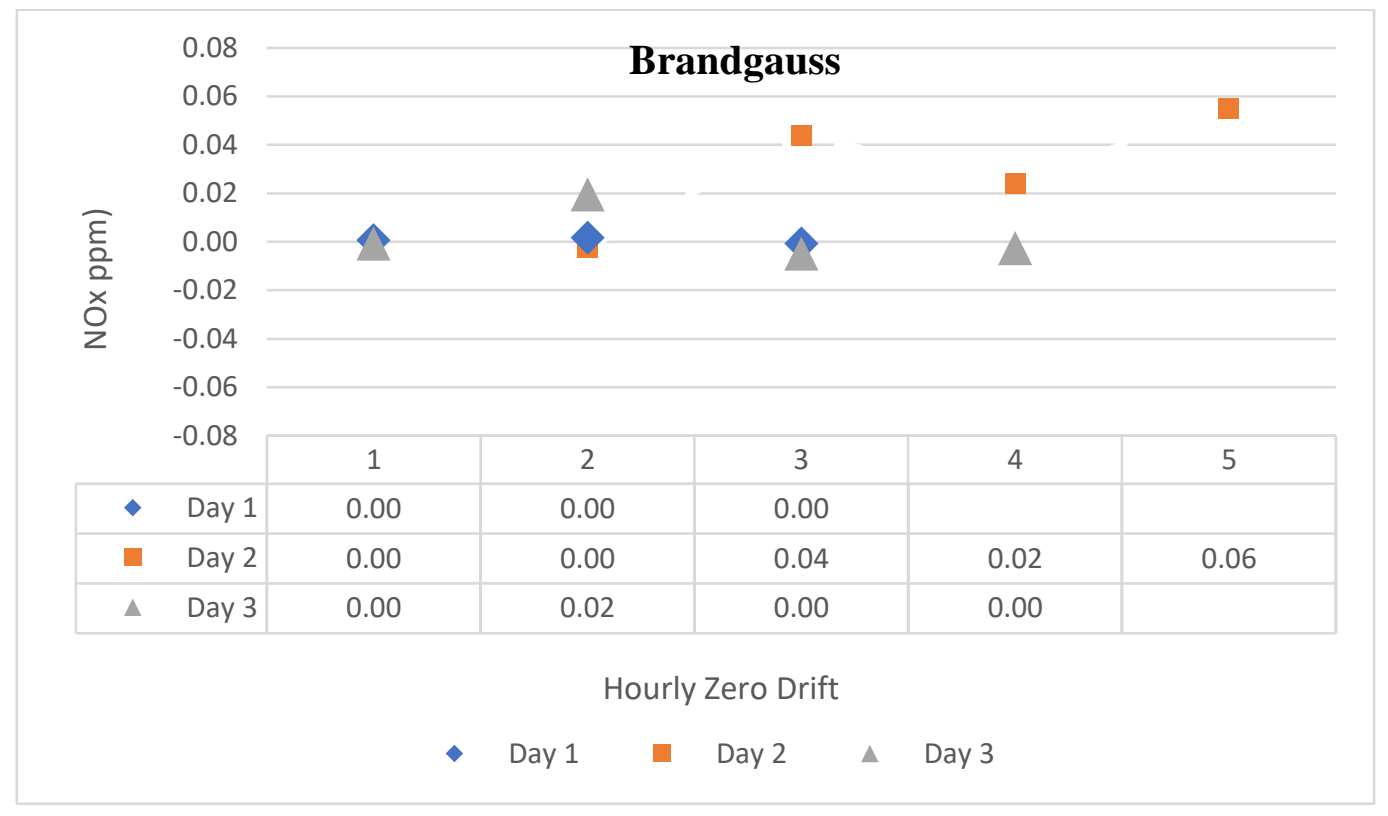

Figure 32- Brandgaus NOx Analyzer Daily Hourly Zero Drift 


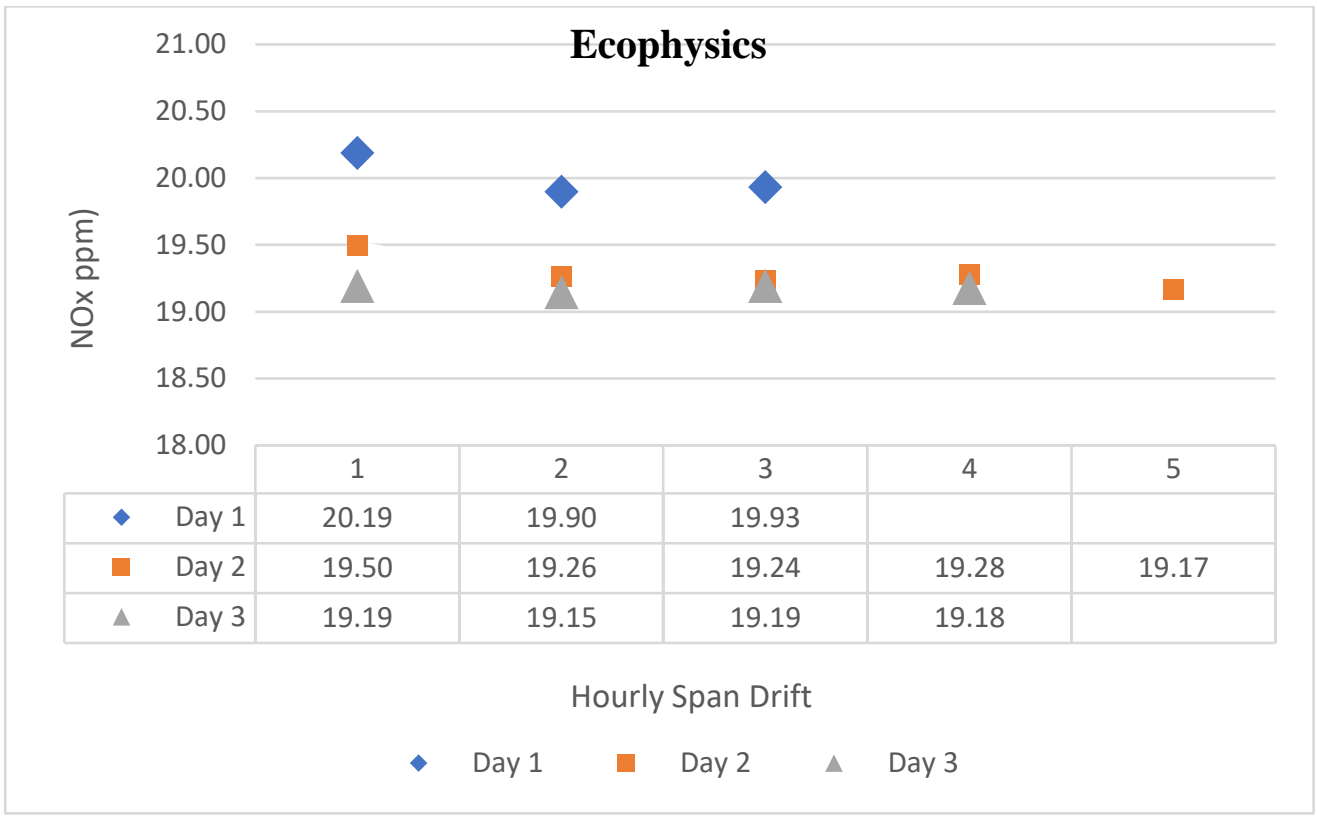

Figure 33- Ecophysics NOx Analyzer Daily Hourly Span Drift

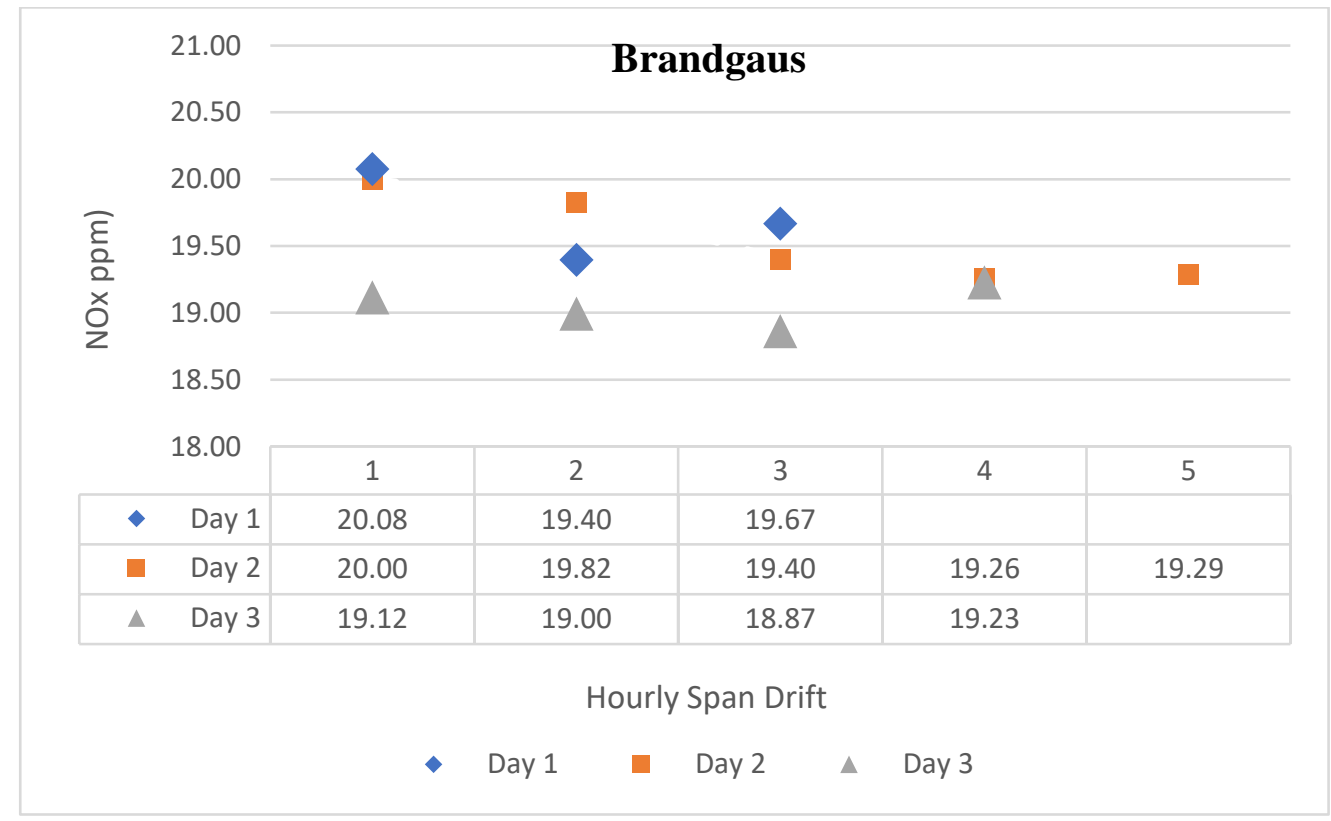

Figure 34- Brandgauss NOx Analyzer Daily Hourly Span Drift 
The hourly drifting pattern of the Ecophysics and Brandgaus analyzer for the 3 days of NOx measurements as shown respectively in Figure 31-34 was different for each day. This might have been as a result of changes in various external conditions (temperature, humidity) during days and time when each test was carried out.

The maximum span drift for the Ecophysics and Brandgaus analyzer was as high as $-2.5 \%$ and $-5.0 \%$ respectively. This was observed on the $2^{\text {nd }}$ hour of the $2^{\text {nd }}$ day for both analyzers, while the overall average span drift across days are $-1.8 \%$ and $-1.6 \%$ for Brandgaus and Ecophysics analyzers respectively. This can be inferred that the measured values overtime by the low NOx analyzers will be lesser than the actual values as a result of drift effect. This drift in low NOx analyzers will be quite significant at near zero level when the NOx measurements from these analyzers are to be used in emission quantification, as the measured value will be lesser than the actual value.

\subsubsection{Linearization Accuracy Comparison}

The low NOx analyzers linearization was carried out with comparison to the FTIR to see their measurement accuracy. A $19.5 \mathrm{ppm}$ NOx and a 20.5 ppm $\mathrm{NO}_{2}$ concentration calibration gases were used for linearization comparison for the analyzers. The values measured by each analyzers were compared to the actual gas concentration as depicted in Figure 37 and Figure 38 in form of percent NOx measured. This showcased possible variation in NOx quantification which might result if the analyzer actually measures either the $\mathrm{NOx}$ or $\mathrm{NO}_{2}$ or a combination of both which was later carried out in section 4.2 .3 of this work. 


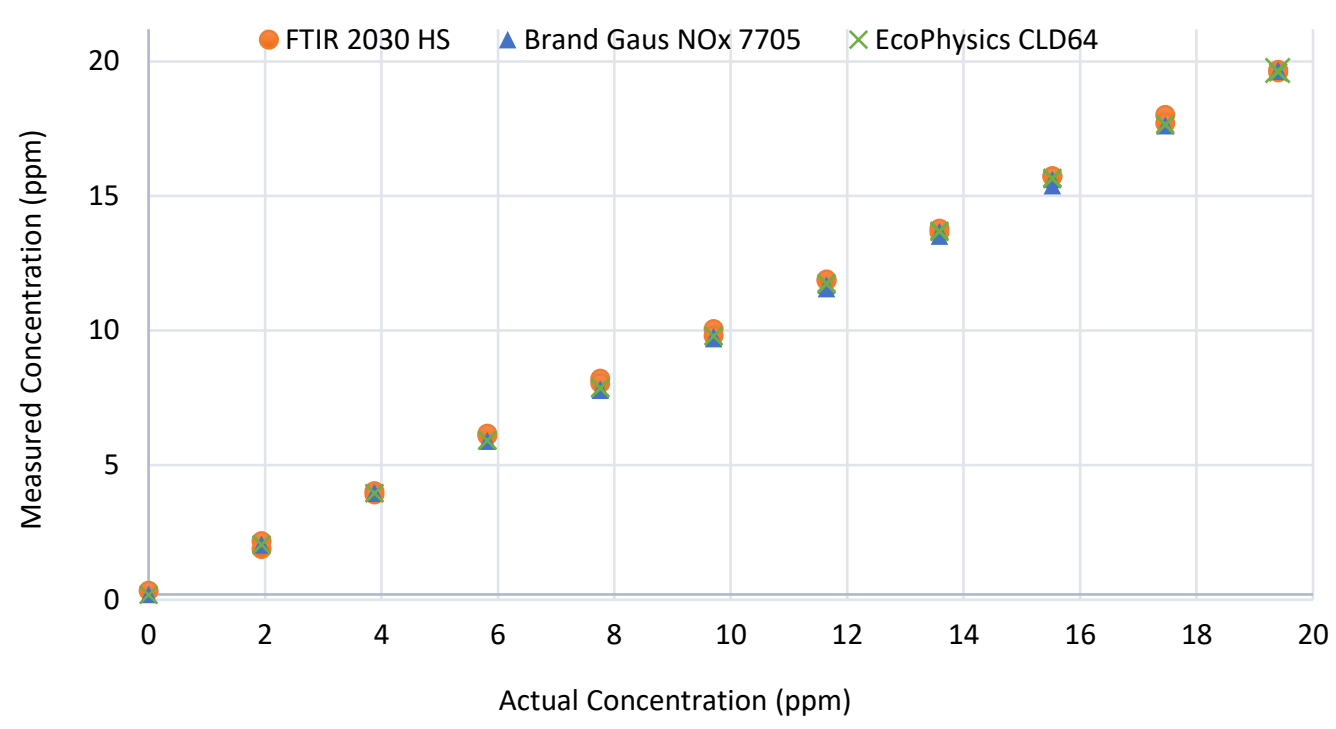

Figure 35- NOx Analyzers Linearization using NOx

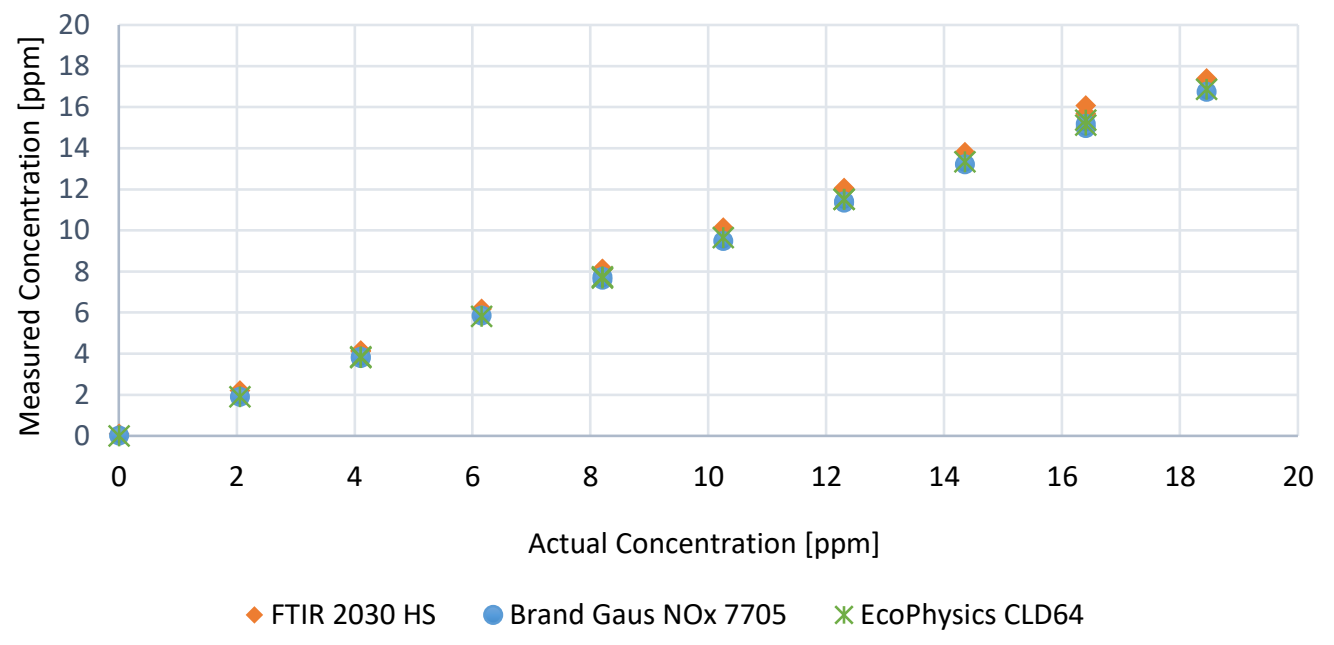

Figure 36-NOx Analyzers Linearization using $\mathrm{NO}_{2}$ 


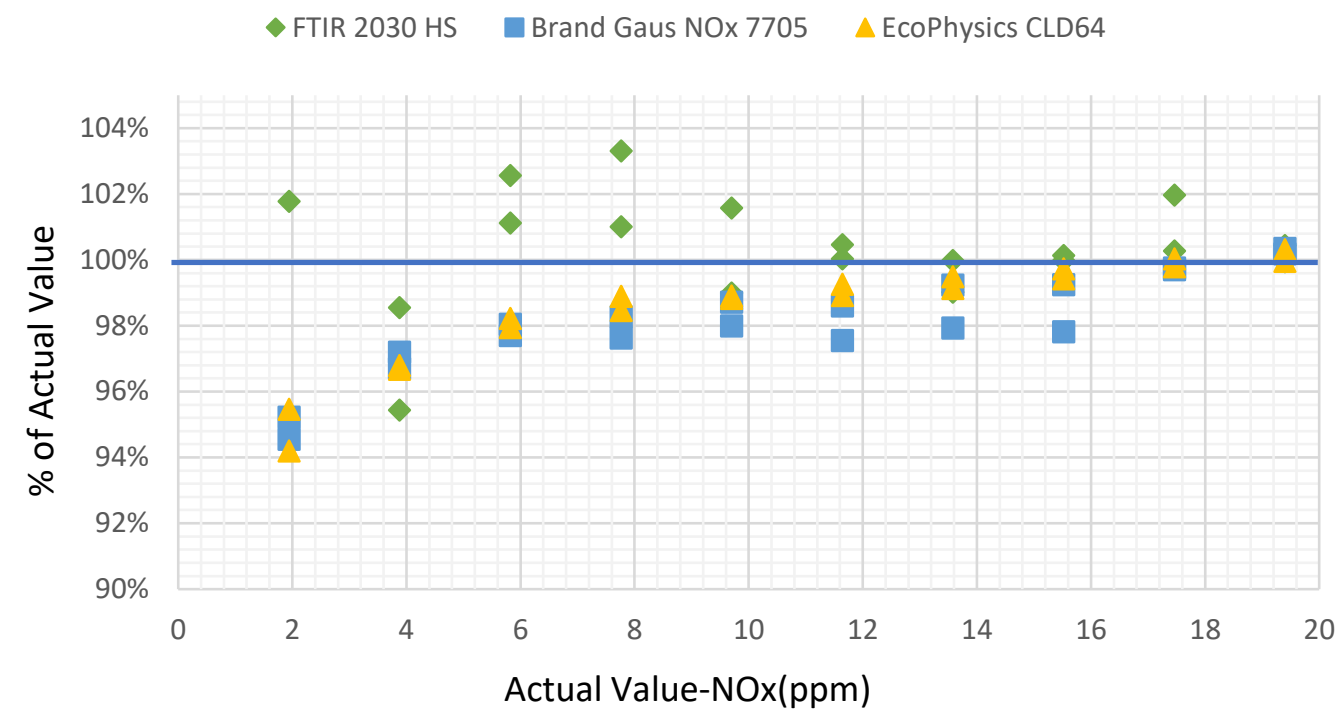

Figure 37- Percent NOx Measured by Analyzer using NOx

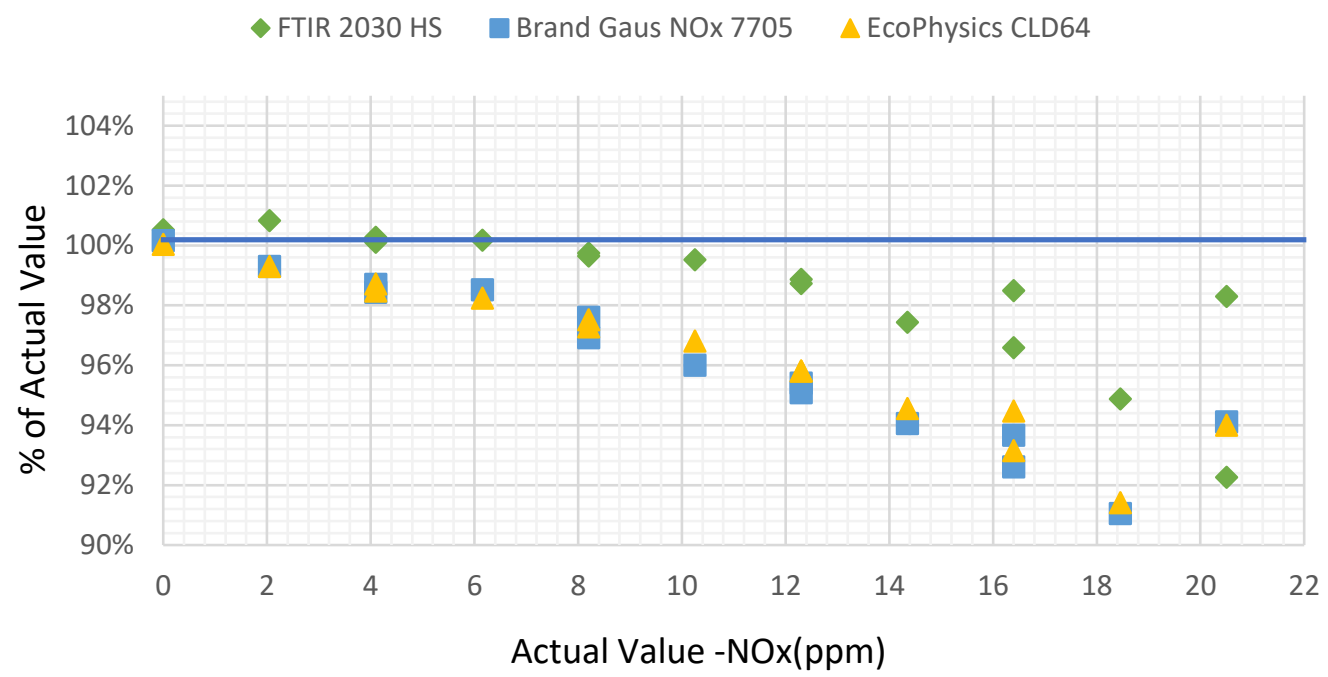

Figure 38- Percent NOx Measured by Analyzer using $\mathrm{NO}_{2}$

The percent of measured NOx is a measure of deviation and inaccuracy of the analyzers to measure the actual $\mathrm{NOx}$ value. The same test was carried out with both $\mathrm{NO}_{2}$ and $\mathrm{NO}_{\mathrm{x}}$ calibration gases measured individual as shown in Figure $37 \& 38$. This is to see the deviation that might results from the $\mathrm{NO}_{\mathrm{x}}$ analyzer measurements as a result of measuring $\mathrm{NOx}$ or $\mathrm{NO}_{2}$ directly. From the results gotten the analyzers measured NOx more accurately at higher concentrations compared to lower concentrations while it was vice versa when $\mathrm{NO}_{2}$ was measured. This is a source of measurement biasness in accurately quantifying NOx at near zero level, since the same CLD measuring principle used in both analyzers gives different concentration measurements for NOx and $\mathrm{NO}_{2}$. 


\subsubsection{Compound NO2 and NOx Linearization}

Compound of $\mathrm{NO}_{2}$ and $\mathrm{NOx}$ was also used for linearization analysis purpose. This was implemented to check the effect of variation in the $\mathrm{NOx}$ and $\mathrm{NO}_{2}$ percentage of the compound, on the accuracy of the low NOx analyzer in measuring total NOx. The $\mathrm{NO}_{2}$ was gradually increased from $0 \%$ while the NOx was concurrently decreased from $100 \%$ and the sum of both equals $100 \%$ at all instance.

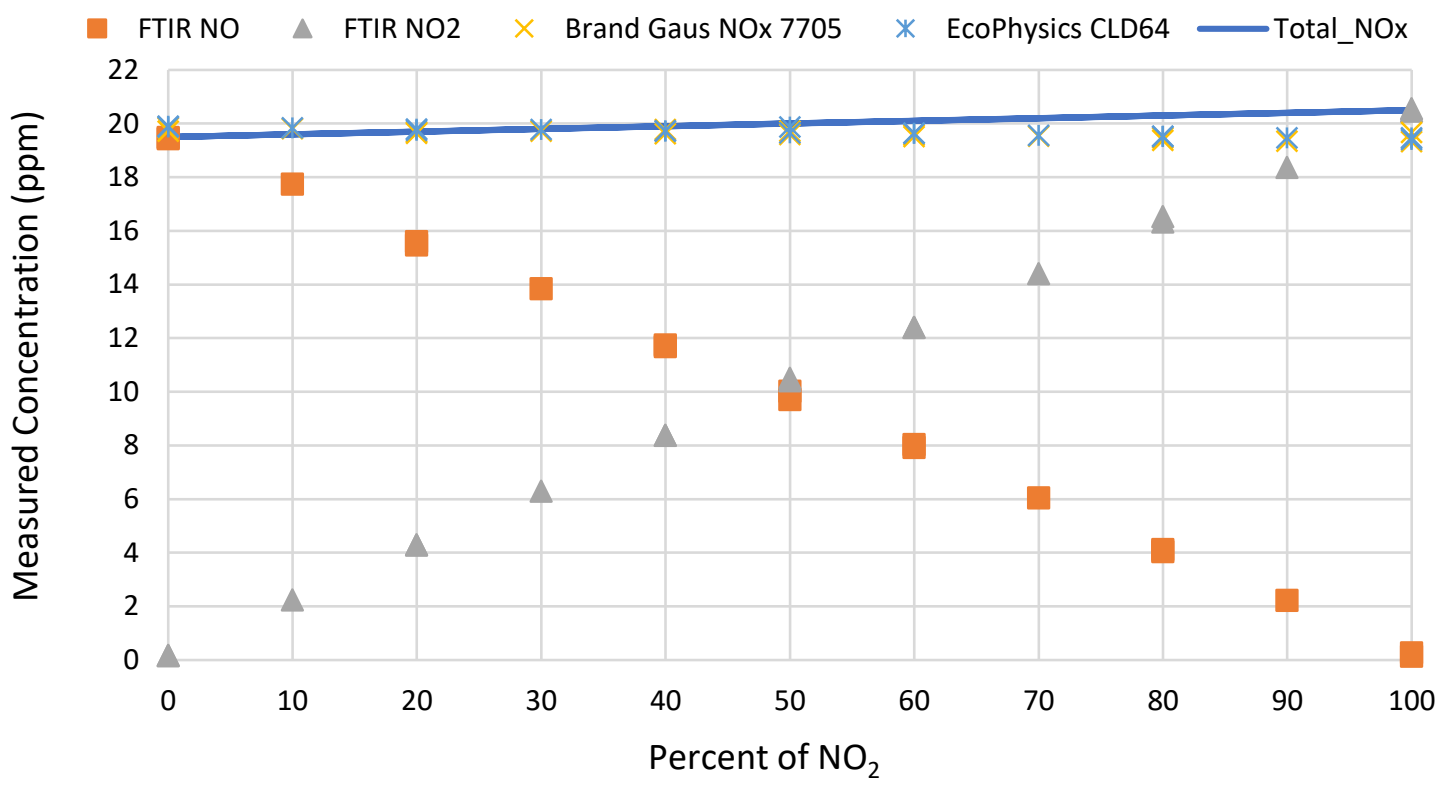

Figure 39- Analyzer Compound $\mathrm{NO}_{2}$ and $\mathrm{NOx}$ linearization accuracy comparison 


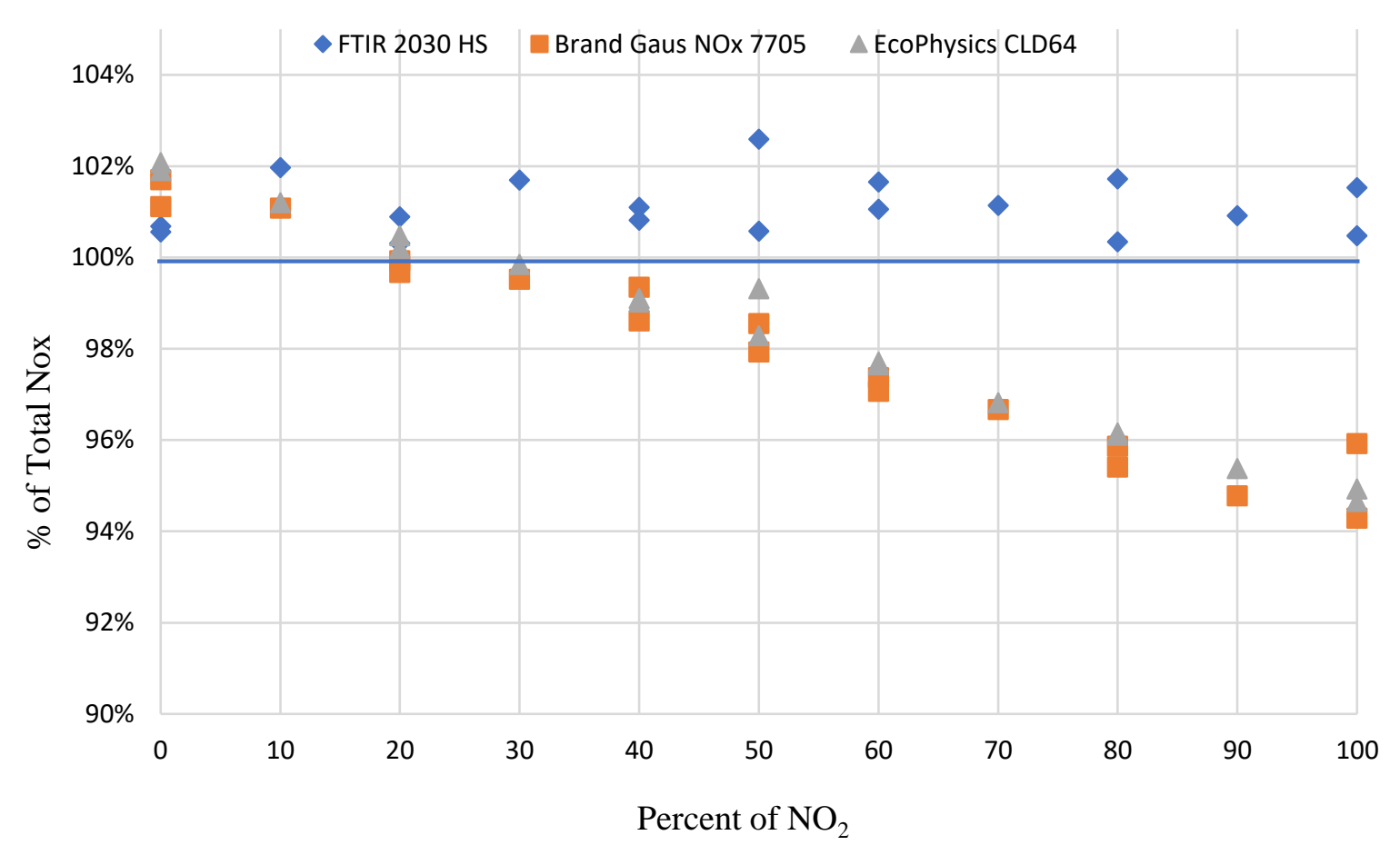

Figure 40- Analyzer Compound $\mathrm{NO}_{2}$ and $\mathrm{NOx}$ linearization Percent Measured

The low NOx analyzers measurements as shown in figure 40 had deviations from the actual total NOx measured values and the variation observed was typically similar to what was obtained for the individual $\mathrm{NOx}$ and $\mathrm{NO}_{2}$ linearization study. The cause of this deviation and apparent similarity between the results gotten for the compound and individual calibration gases NOx measurements will require further studies to be determined. As this might be ascribed to the measuring instruments error or possibly other factors beyond the scope of this work. At lower measurement ranges the effects of this deviations will be consequential in accurately measuring NOx using the CVS sampling system.

\subsubsection{Linearization Verification}

The linearization verification for both Brandgaus and Ecophysics analyzers was carried out according to the EPA "CFR, Title 40, Part 1065.307" standards for measurement systems. In which gas analyzers for laboratory testing is included among those instruments requiring linearity verifications. 
The least square regression of measurements obtained from both analyzers during linearization was utilized in computing the linearization verification.

Table 11- Summary of Analyzers Measurements Least Square Regression

\begin{tabular}{|c|c|c|}
\hline Parameter & Ecophysics & Brandgaus \\
\hline X_min & 0 & 0 \\
\hline X_Max & 19.4 & 19.4 \\
\hline $\mathrm{a}_{0}$ & -0.12703 & -0.13757 \\
\hline $\mathrm{a}_{1}$ & 1.0048 & 1.0013 \\
\hline SEE & 0.001613 & 0.003803 \\
\hline $\mathrm{R}^{2}$ & 1 & 1 \\
\hline
\end{tabular}
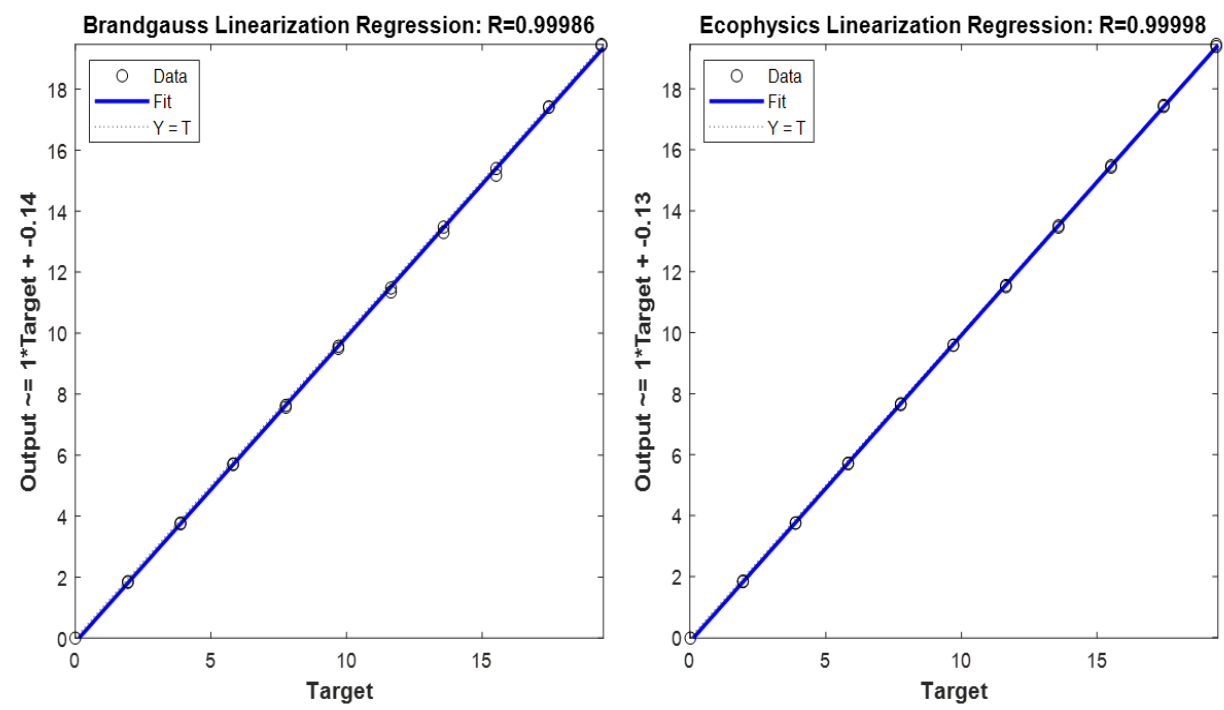

Figure 41 - Analyzers Linear Regression Plot

Table 12- Summary of Analyzer Linearization Verification

\begin{tabular}{|c|c|c|c|c|}
\hline $\begin{array}{c}\text { Linearization Parameter } \\
\text { (Gas analyzers for laboratory testing) }\end{array}$ & $\begin{array}{c}\text { X_min(a1-1) + a0 } \\
(\leq 0.5 \% \text { X_max })\end{array}$ & $\mathbf{a} 1$ & $\begin{array}{c}\text { SEE } \\
(\leq 1 \% \text { X_max })\end{array}$ & $\begin{array}{c}\mathbf{R}^{2} \\
(\geq)\end{array}$ \\
\hline $\begin{array}{c}\text { EPA “CFR, Title 40, Part 1065.307 } \\
\text { (Study Specific) }\end{array}$ & 0.097 & $0.99-1.01$ & 0.194 & 0.998 \\
\hline Ecophysics & -0.12703 & 1.0048 & 0.001613 & 1 \\
\hline Brandgaus & -0.13757 & 1.0013 & 0.003803 & 1 \\
\hline
\end{tabular}


From Table 12 both low NOx gas Analyzers pass the linearization verification test according to the EPA standards for gas analyzers used for laboratory testing.

\subsection{Virtual NOx Injection}

Error in the recovered mass of NOx to the mass of NOx injected varied for each sampling conditions at which virtual NOx injection (VNI) was carried out. The sampling conditions as shown in showcased much error was observed when both the tunnel and the analyzer sampling system were both heated compared to when either of both was heated. The rationale behind the first condition of not heating the analyzer sampling lines was to check possible errors in NOx measurement that might result due to faulty heated line along the sampling system.

The recovered NOx mass error should not be greater than $2 \%$ according to the EPA 40 CFR 1065 standards and this error went as high as $-10 \%$ for this study. This showcased according to this study that the error associated with accurate quantification of NOx increased when heated is added and temperature increases.

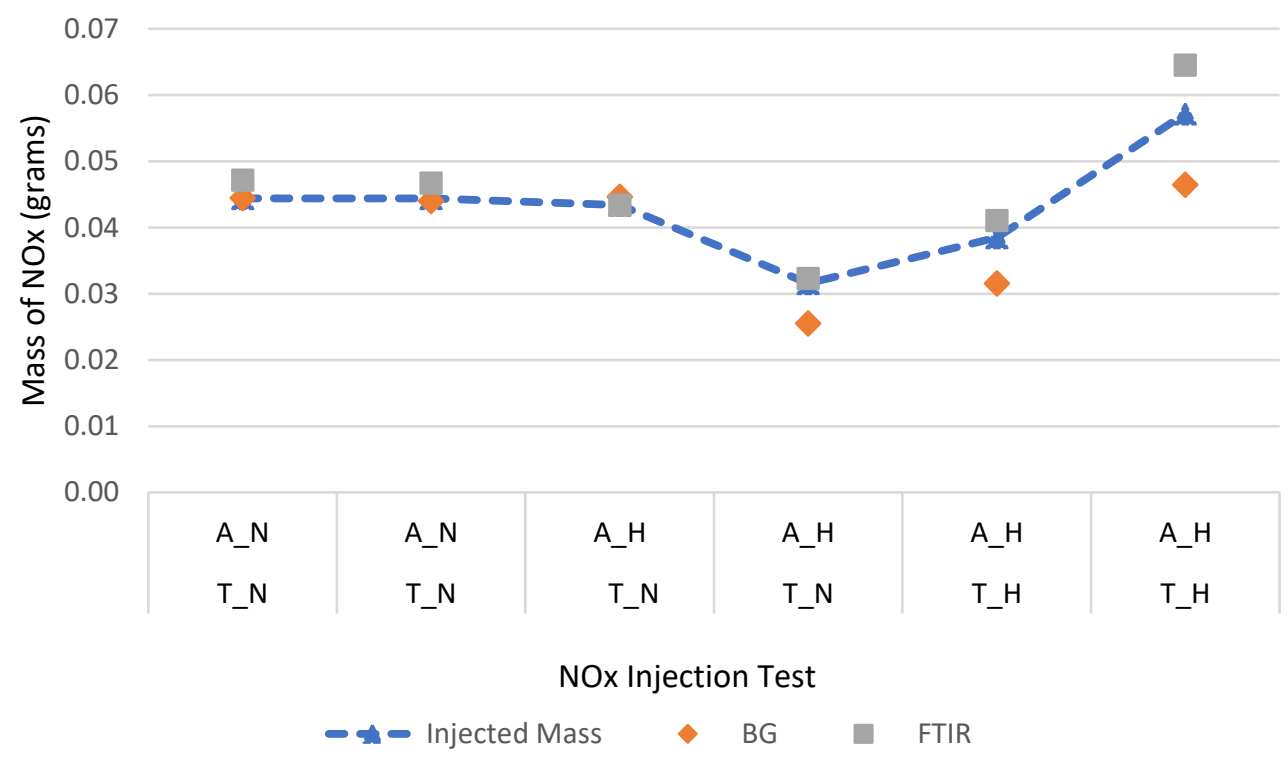

A_N= Analyzer Sampling not Heated, A_H=Analyzer Sampling Heated, T_N= Tunnel not Heated , T_H=Tunnel Heated

Figure 42- Comparison of Injected Mass of NOx to Recovered Mass of NOx 


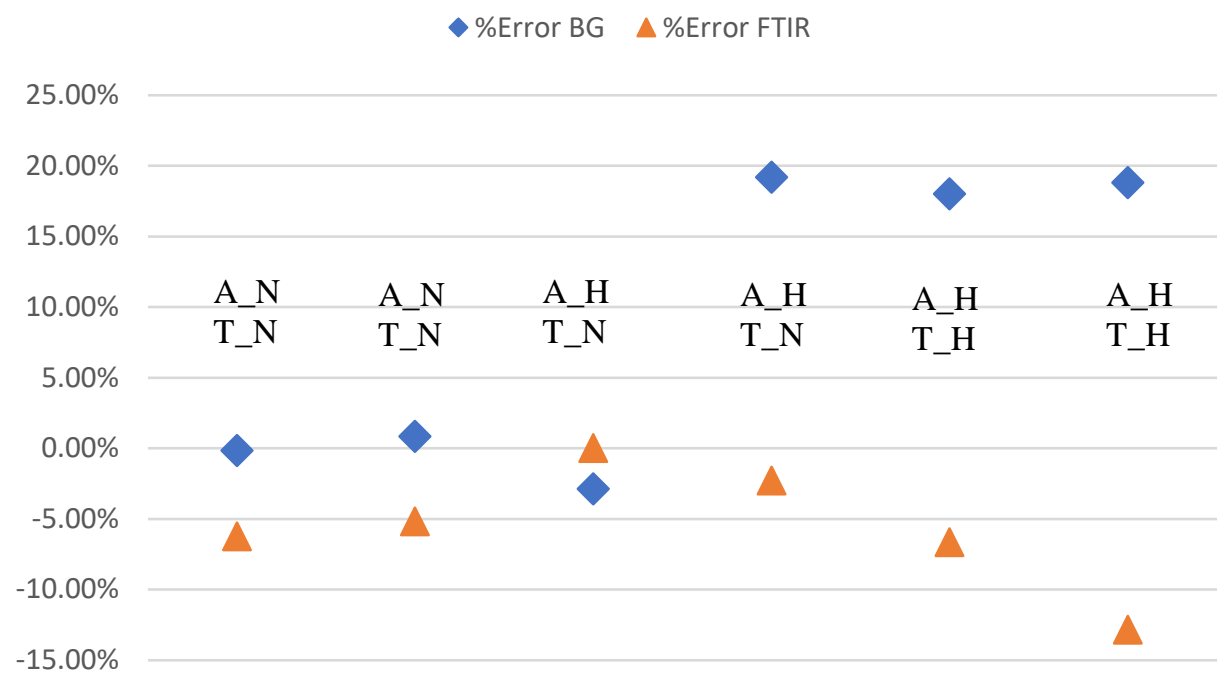

A_N= Analyzer Sampling not Heated, A_H=Analyzer Sampling Heated, T_N= Tunnel not Heated , T_H=Tunnel Heated

Figure 43- Percent Error in Recovered Mass of NOx.

The reason for this under-recovery and over-recovery in measured NOx value when injected virtually cannot be ascertain. It was showcased in this study that the integrity of this CVS measurement system in quantifying NOx from engine or other variable sources might be questionable. As the quantification of a known mass of NOx resulted in high errors when sampling temperature and heating increased. Since this high temperature range will be a typical scenario of an exhaust gas, it can be inferred that inaccurate measurement is bound to increase if the CVS system is used to quantify NOx especially at near zero level.

This error might be as a result of increase in the solubility of NOx due to increased temperature which would have resulted in the loss of NOx along the sampling system. It might also possibly be due to measuring device error at higher sampling temperature. The reason for this inaccuracy will require further studies to ascertain how the error percent of the recovered NOx mass increases with the heating of the tunnel and the sampling systems.

\subsection{Tunnel Heating}

The tunnel heating results showcased a stable water temperature reading along the 12 locations where the thermocouples were placed. These values as shown in Table 13 were all above $35^{\circ} \mathrm{C}$, which is a safe temperature to avoid water condensation and cold spot along the CVS tunnel. 
Table 13 - Water Temperature across the Heated Tunnel

\begin{tabular}{|l|l|l|l|l|}
\hline Thermocouple Location & Top & $\begin{array}{l}\text { Mid-Section } \\
\text { Front }\end{array}$ & $\begin{array}{l}\text { Mid-Section } \\
\text { Back }\end{array}$ & Bottom \\
\hline Inlet $\left({ }^{\circ} \mathrm{C}\right)$ & 54.0 & 57.0 & 53.4 & 52.9 \\
\hline Middle $\left({ }^{\circ} \mathrm{C}\right)$ & 58.9 & 57.8 & 51.5 & 51.3 \\
\hline Outlet $\left({ }^{\circ} \mathrm{C}\right)$ & 56.8 & 57.2 & 49.5 & 50.4 \\
\hline
\end{tabular}

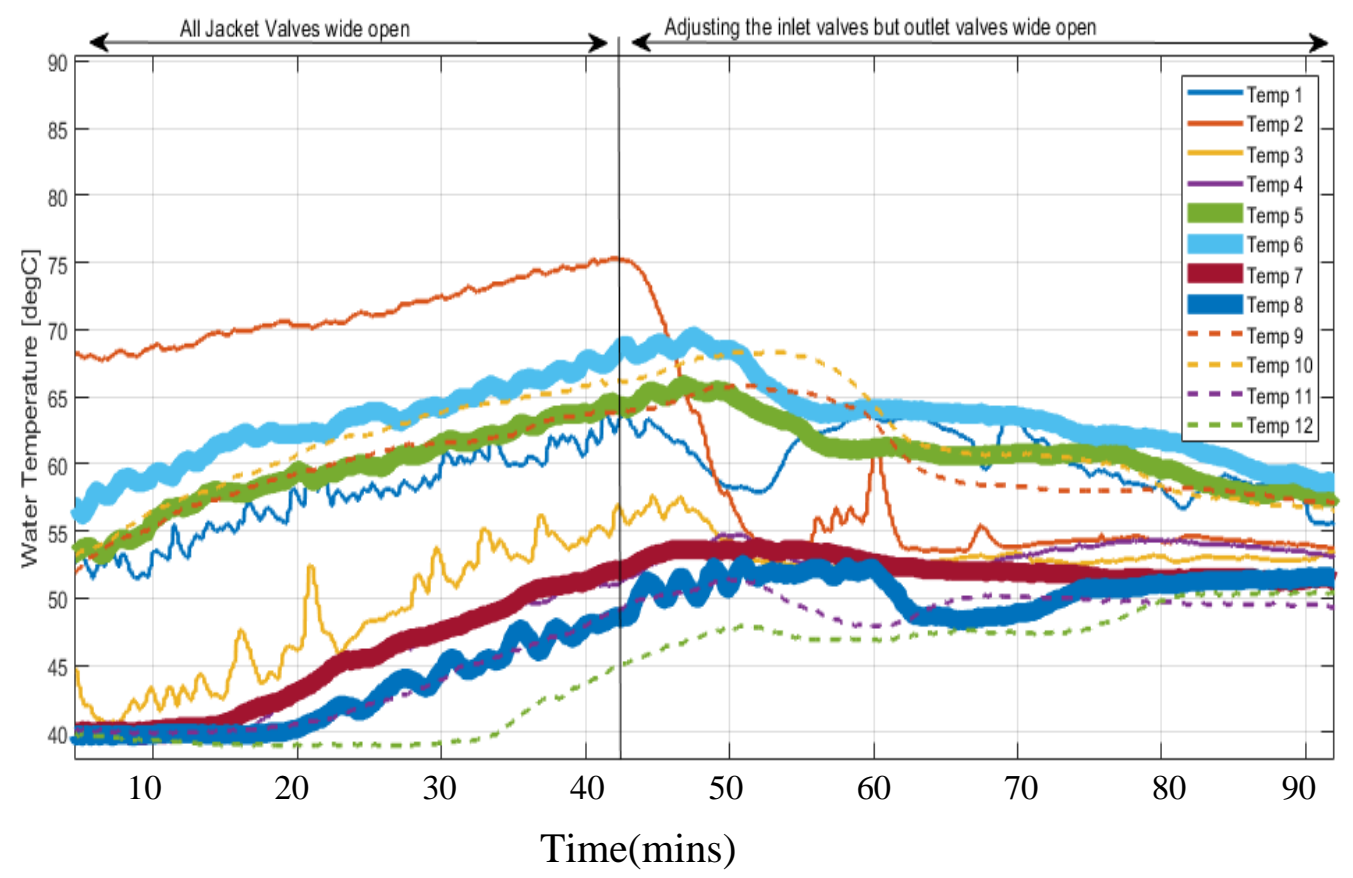

Figure 44 - Optimization of Tunnel Heating to minimize temperature difference

The tunnel heating was optimized to ensure minimal temperature differences along portion of the wall thereby avoiding cold spots. The thermocouples water temperature readings were used in the optimization process to ensure the CVS tunnel wall temperature might be along close range. From the results gotten the tunnel heating increased the air temperature while decreasing the relative humidity of the dilution air. The lower air temperature at the inlet of the tunnel was raised from $17^{\circ} \mathrm{C}$ to a stable temperature of $22^{\circ} \mathrm{C}$. While the relative humidity of the air was decreased to $60 \%$. 


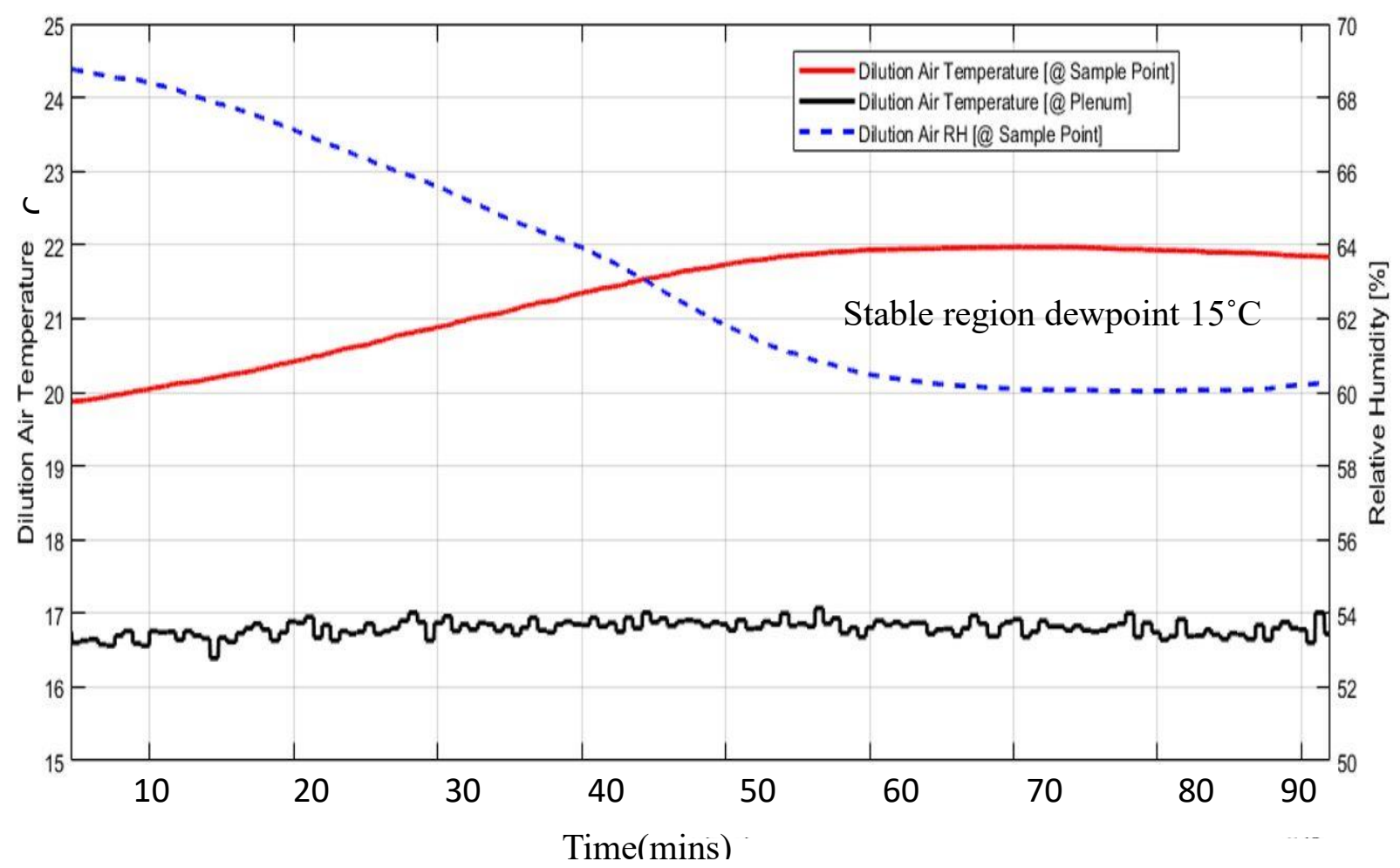

Figure 45 - Effect of heating on Relative Humidity and Temperature at Sample Plane adapted from [61]

Temperature and relative humidity of the dilution air achieved stability after heating up the tunnel. This stability is certain to aid in reducing loss of NOx associated with cold spots in the CVS tunnel. This will minimize hang-ups effect and condensation of exhaust water vapor. Reducing these presumed effects will aid accurate quantification of NOx as loss of NOx at these low levels are quite significant for correct emission measurement. 


\section{Conclusions and Recommendations}

\subsection{Conclusions}

At the end of the study the factors which are susceptible to affect the ability to accurately quantify and characterize NOx emissions at near Zero level were investigated according to the objectives of this work.

$>$ There exists variation in ambient NOx and THC concentration within 10 minutes interval.

$>$ The dilution air filtration system was more effective in reducing THC compared to NOx though the average NOx reduction was $47 \%$ and $14.3 \%$ as measured by the Ecophysics and Brandgaus analyzers respectively. While for the THC as measured by MEXA was $5 \%$ reduction.

$>$ THC reduction efficiency increased with increased temperature and decreased with increased relative humidity

$>$ An overall average analyzer drift of $-1.8 \%$ and $-1.6 \%$ was recorded for Ecophysics and Brandgaus analyzers respectively.

$>$ The Brandgauss and Ecophysics NOx analyzers both passed the linearization verification test according to the EPA "CFR, Title 40, Part 1065.307 standards.

$>$ A stable air temperature of $22^{\circ} \mathrm{C}$ and a stable reduction in relative humidity to $60 \%$ was observed at the sampling plane as a result of tunnel heating

$>$ The variation of the NOx concentration in ambient air used for exhaust dilution in the CVS tunnel according to this study occurred within 10 minutes interval and this fluctuation in measured NOx were typically affected by human activities which increased the NOx concentration of the environment.

$>$ The dilution air filter used according to this study was more relatively effective in reducing THC than NOx concentration. 


\subsection{Recommendations}

This study has been able to show some factors that can lead to possible errors resulting in inaccuracies in measuring NOx at near Zero level. The recommendations from this work includes:

- Dilution air filtration study, pre and post sampling should be done concurrently to be able to account for possible variations in NOx concentration within 600seconds

\subsection{Future Works}

- Further study is required to determine and understand the causes and reasons for the errors and variations observed while investigating some of the following selected factors in this study:

○ Analyzer Drift

- Dilution air filter loading

- Proportional Bag Sampling mass flow controller rapid adjustment with respect to varying flowrate

- Impact of pressure pulsation during sampling on CVS measurement

- A need to quantify each error associated to each study and seek means to reduce this error and their cause.

- Overall error propagation calculation is required to identify sub-components of the CVS system contributing significant error 


\section{References}

[1] "Low Level NOX Measurements and Other Compliance Issues on Gas Turbine Combined Cycle Units." [Online]. Available: http://www.rmb-consulting.com/san/lownox.htm. [Accessed: 08-Feb-2019].

[2] T. C. Austin and L. S. Caretto, "Improving the Calculation of Exhaust Gas Dilution During Constant Volume Sampling," SAE Tech. Pap., no. 724, 1998.

[3] A. Majerczyk and S. Radzimirski, "Analysis of Measurement Errors of CVS method used for tests of pollutants emissions from light duty vehicles," J. KONES. Powertrain Transp., vol. 20, no. 4, pp. 253-259, 2015.

[4] A. Smith and H. Davies, "“A review of the history of emission legislation, urban and national transport trends and their impact on transport emissions," Trans. Built Environ., vol. 23 , no. 17433509 , pp. 293-302, 1995.

[5] United States Environmental Protection Agency, "Emission Standards Reference Guide for Nonroad Engines and Vehicles," 2013. [Online]. Available: https://www.epa.gov/emissionstandards-reference-guide. [Accessed: 27-Feb-2019].

[6] A. P. Thiruvengadam, "Evaluation of Exhaust After-treatment Device Effectiveness in Reducing Regulated and Unregulated Emissions from Natural Gas Fueled Heavy Duty Transit Bus," West Virginia University, 2008

[7] USEPA, “NEI Report Dashboard.’'[Online]. Available:

https://edap.epa.gov/public/extensons/nei_report_2014/dashboard.html\#trend-db. [Accessed:11-Feb-2019].

[8] CARB, "Heavy-Duty Low NOx.” [Online]. Available:

https://www.arb.ca.gov/msprog/hdlownox/hdlownox.htm. [Accessed: 12-Feb-2019].

[9] US EPA “Memorandum in Response to Petition for Rulemaking to Adopt Ultra-Low NOx Standards for On-Highway Heavy-Duty Trucks and Engines (December 20, 2016),” 2024.

[10] CARB, "Optional Reduced NOx Emission Standards for On-Road Heavy-duty Engines,’2014.[Online]Available https://www.arb.ca.gov/msprog/onroad/optionnox/optionnox.htm. [Accessed: 12-Feb2019].

[11] K. Johnson, "Ultra-Low NOx Natural Gas Vehicle Evaluation,” 2016.

[12] US EPA to update NOx emission standards for heavy-duty engines," 2018. [Online]. Available: https://www.dieselnet.com/news/2018/11epa.php. [Accessed: 13-Feb-2019].

[13] Conference Report: SAE HDD Emissions Control Symposium,” 2018. [Online]. Available: https://www.dieselnet.com/news/2018/10sae.php. [Accessed: 13-Feb-2019].

[14] C. Görsmann, "SAE 2014 Heavy-Duty Diesel Emissions Control Symposium,” Johnson Matthey Technol. Rev., vol. 59, no. 2, pp. 139-151, Apr. 2015. 
[15] E. M. Petersen Publishing Company. and E. M. Rosen, The Petersen automotive troubleshooting repair manual. New York: Grosset \& Dunlap, 1975.

[16] A. M. Williams, "Lean NOx Trap Catalysis for Lean Burn Natural Gas Engines," University of Tennesse, 2004.

[17] T. T. Morita, N. Suzuki, N. Satoh, K. Wada, and H. Ohno, "Study on Low NOx Emission Control Using Newly Developed Lean NOx Catalyst for Diesel Engines," SAE Int., 2007.

[18] H. Kojima et al., "Next Generation All in One Close-Coupled Urea-SCR System," SAE Tech. Pap., no. 2015-01-0994, 2015.

[19] M. A. Gonzalez D and D. Di Nunno, "Internal Exhaust Gas Recirculation for Efficiency and Emissions in a 4-Cylinder Diesel Engine," 2016.

[20] M. Baratta, R. Finesso, D. Misul, and E. Spessa, "Comparison between Internal and External EGR Performance on a Heavy Duty Diesel Engine by Means of a Refined 1D Fluid-Dynamic Engine Model," SAE Int. J. Engines, vol. 8, no. 5, pp. 2015-24-2389, Sep. 2015.

[21] K. Moore, “Testing Automotive Exhaust Emissions,” 2013.

[22] M. A. Michael A. Plint and A. Martyr, Engine testing : theory and practice. ButterworthHeinemann, 1995.

[23] S. D. Shah, A. Mauti, J. F. O. Richert, M. J. Loos, and R. E. Chase, "Measuring NOx in the Presence of Ammonia," SAE Tech. Pap., vol. 01, no. 0331, Apr. 2007.

[24] Cambustion, "Chemiluminescence detector (CLD) principle," Cambustion Ltd. [Online]. Available: http://www.cambustion.com/products/cld500/cld-principles. [Accessed: 18Feb-2019].

[25] Cambustion, "Nondispersive Infrared Detector (NDIR) principle.” [Online]. Available: https://www.cambustion.com/products/ndir500/operating-principle.[Accessed:19-Feb2019].

[26] Cambustion, "Flame Ionization Detector (FID) principle." [Online]. Available: https://www.cambustion.com/products/hfr500/fast-fid-principles.[Accessed:19-Feb-2019]

[27] Spiraxsarco, "Flowmetering." [Online]. Available: http://www.spiraxsarco.com/global/ uk/Products/Documents/FINALFlowmetering_white_paper.pdf. [Accessed: 16-Feb-2019].

[28] A. Shrivastava and V. Gupta, "Methods for the determination of limit of detection and limit of quantitation of the analytical methods," Chronicles Young Sci., vol. 2, no. 1, p. 21, 2011.

[29] T. Michael, S. Ellison; and R. Wood; "Harmonized Guidelines for Single-Laboratory Validation of Methods of Analysis," Pure Appl. Chem, vol. 74, no. 5, pp. 835-855, 2002.

[30] R. Wood and J. Thornton, “ATMS 451 Lab Manual: Time Response.

[31] U.S Government Publishing Office, "Electronic Code of Federal Regulations- 40CFR Part 1065.’[Online]. Available: https://www.ecfr.gov/. [Accessed: 18-Mar-2019]. 
[32] U.S. Government Publishing Office, "Electronic Code of Federal Regulations 40CFR Part 60.[Online]. Available: https://www.ecfr.gov/. [Accessed: 16-Feb-2019].

[33] P. Scott, "Uncertainty in measurement: Noise and how to deal with it," in Intermediate lab manual, 2000.

[34] Franck Dernoncourt, "What is the basic difference between noise and outliers in Data mining? -Quora," 2013. [Online]. Available: https://www.quora.com/What-is-the-basicdifference- between-noise-and-outliers-in-Data-mining. [Accessed: 17-Feb-2019]. .

[35] Soft computing and intelligent information systems (SCI2S), "Noisy Data in Data Mining Soft Computing and Intelligent Information Systems," sci2s.ugr.es. [Online]. Available: http://sci2s.ugr.es/noisydata. [Accessed: 17-Feb-2019].

[36] D. Johnson, “Signal-to-noise ratio,” Scholarpedia, vol. 1, no. 12, p. 2088, 2006.

[37] “J. Burke, "Signal -to-noise ratio(S/N or SNR)." [Online]. Available: https:// searchn etworking .techtarget.com/definition/signal-to-noise-ratio. [Accessed: 17-Feb-2019].

[38] R. Williams, "Terminology used in instrument accuracy," in American school of gas measurement technology, 2003, pp. 279-84.

[39] J. J. Carr and J. M. (John M. Brown, Introduction to biomedical equipment technology. Prentice Hall, 1998.

[40] Hershal,"Resolution and Sensitivity - What is measurement instrument or gage 'Sensitivity?'’[Online]. Available: https://elsmar.com/elsmarqualityforum/threads/ resolution-and-sensitivity-what-is-measurement-instrument-or-gage-sensitivity.11380/. [Accessed: 18-Mar-2019].

[41] A. Joseph and T. F. Kamil, "Anybody can tell me the practical aspects of selectivity, sensitivity and cross-sensitivity of a sensor?'[Online]. Available:https: //www. researchgate.net/post/Anybody_can_tell_me_the_practical_aspects_of_selectivity _sensitivity_and_cross-sensitivity_of_a_sensor. [Accessed: 18-Mar-2019].

[42] M. Gautam, "In--Use, On--Road Emissions Testing of Road Emissions Testing of HeavyDuty Diesel Vehicles: Challenges and Opportunities," 2015. [Online]. Available: https://www.epa.gov/sites/production/files/2015-03/documents/02132002mstrs_gautam. pdf. [Accessed: 26-Feb-2019].

[43] Legal Information Institute Cornell Law School, "Electronic Code of Federal Regulations 40 CFR1065.307 - Linearity verification." [Online]. Available:https:/www.law .cornell.edu/cfr/text/40/1065.307. [Accessed: 19-Feb-2019].

[44] V. Petrović, Z. Bracanović, B. Grozdanić, S. Petrović, S. Sazhin, and D. Knežević, “The design of a full flow dilution tunnel with a critical flow venturi for the measurement of diesel engine particulate emission," FME Trans., vol. 43, no. 2, pp. 99-106, 2015.

[45] P. Lijewski, P. Fuć, A. Ziółkowski, Ł. Rymaniak, and J. Merkisz, "Measurement of Exhaust Emissions under Actual Operating Conditions with the Use of PEMS: Review of Selected Vehicles," in Improvement Trends for Internal Combustion Engines, InTech, 2018.

[46] H. Burtscher, "Tailpipe Particulate Emission Measurement for Diesel Engines," 2001. 
[47] D. R. Cocker, S. D. Shah, K. Johnson, J. W. Miller, and J. M. Norbeck, "Development and Application of a Mobile Laboratory for Measuring Emissions from Diesel Engines. 1. Regulated Gaseous Emissions," Environ. Sci. Technol., vol. 38, no. 7, pp. 2182-2189, 2004.

[48] M. C. Besch, "In-line, Real-time Particulate Matter Sensors for OBD and Exhaust Aftertreatment System Control Applications,” West Virginia University, 2016.

[49] Legal Information Institute Cornell Law School, "Electronic Code of Federal Regulations 40 CFR 90.420 - CVS concept of exhaust gas sampling system.” [Online]. Available:

https://www.law.cornell.edu/cfr/text/40/90.420. [Accessed: 23-Feb-2019].

[50] D. R. Cocker, S. D. Shah, K. C. Johnson, X. Zhu, J. W. Miller, and J. M. Norbeck, "Development and Application of a Mobile Laboratory for Measuring Emissions from Diesel Engines. 1. Regulated Gaseous Emissions Motivation for Mobile Emissions Laboratory," Environ. Sci. Technol., vol. 38,no. 24, pp. 2182-2189, 2004.

[51] Legal Information Institute Cornell Law School, "Electronic Code of Federal Regulations 40 CFR 90.424 - Dilute sampling procedures - CVS calibration." [Online]. Available:https://www.law.cornell.edu/cfr/text/40/90.424. [Accessed: 18-Mar-2019].

[52] Legal Information Institute Cornell Law School, "Electronic Code of Federal Regulations 40 CFR 1065.341 - CVS, PFD, and batch sampler verification (propane check). [Online]. Available: https://www.law.cornell.edu/cfr/text/40/1065.341. [Accessed: 26-Feb-2019]..

[53] M. M. Maricq, R. E. Chase, R. Vogt, and V. Scheer, "Constant Volume Rapid Exhaust Dilution,"2002."

[54] W. N. Mcmahon, D. Peterson, E. I. Sun, A. J. Dallas, E. Stenersen, and J. Wong, "Evaluation of an Enhanced Constant Volume Sampling System and a Bag Mini Diluter for Near Zero Exhaust Emission Testing," SAE Tech. Pap. Ser., vol. 1, no. 724, 2010.

[55] W. M. Silvis and R. E. Chase, "Proportional Ambient Sampling: A CVS Improvement for ULEV and Lean Engine Operation," SAE Transactions, vol. 108. SAE International, pp. 129-139, 1999.

[56] C. H. Frey, Quantification of variability and uncertainty in emission factors and emission inventories. 2015.

[57] T. A. Nevius and J. J. Gibbs, "Further Investigation of Losses in NOx Recovery with a CVS,” SAE Tech. Pap. Ser., vol. 1, no. 724, 2010.

[58] D. McDonnough, T. A. Nevius, and D. Whelan, "Investigation of Errors in NOx Recovery with a CVS Using a Vehicle Exhaust Emission Simulator (VEES) as a Cross Check Tool," SAE Tech. Pap. Ser., vol. 1, no. 724, 2010.

[59] T. W. Van Der Mark, E. Kort, R. J. Meijer, D. S. Postma, and G. H. Koëter, "Water vapour and carbon dioxide decrease nitric oxide readings," Eur Respir J, vol. 10, pp. 2120-2123, 1997.

[60] B. Mace, "Measurement Challenges for Modern Heavy Duty Engines," 2016.

[61] Air Filters, "Dilutiuon Air filter Schematic." [Online]. Available: www.airfilterusa.com.

[61] WVU CAFEE ULNOX Study, Presentation .2018 


\section{Appendix}

\section{$\underline{\text { Background Variability }}$}

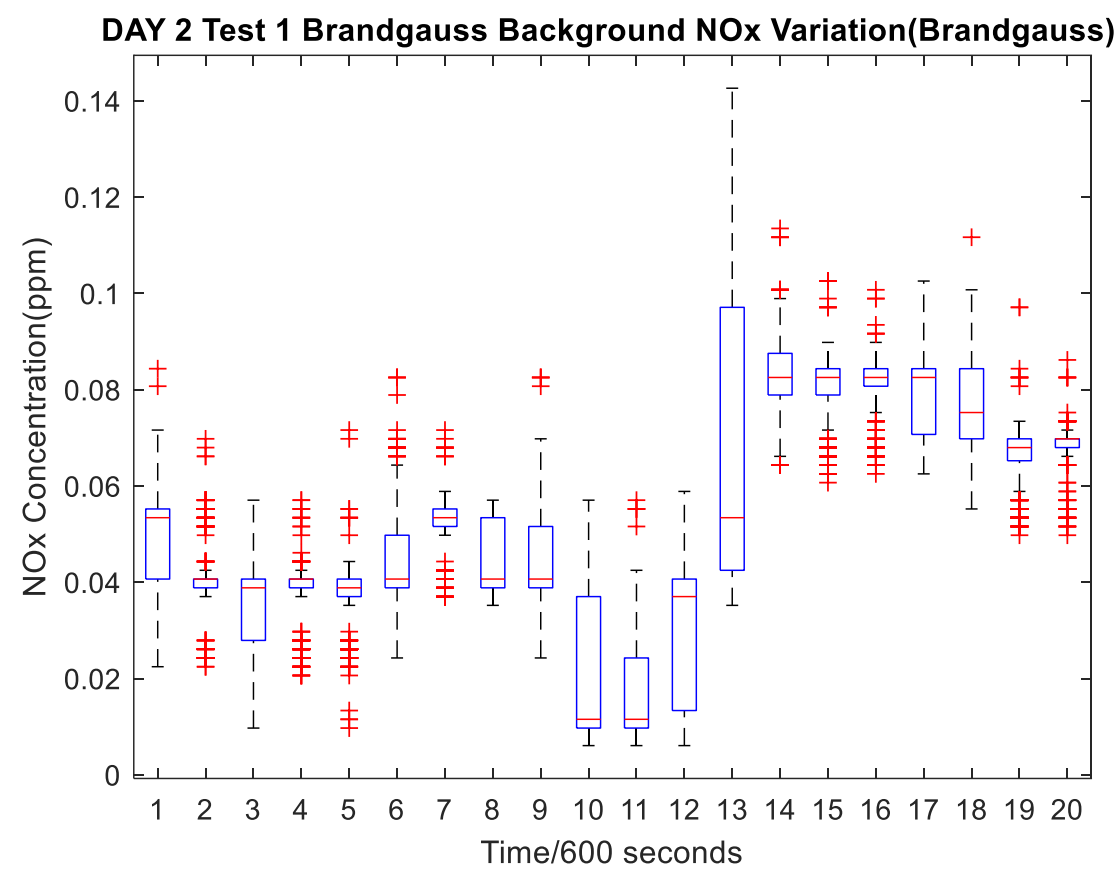

Figure A1 - Day 2 Test 1 Background NOx Variation(Brandgauss)

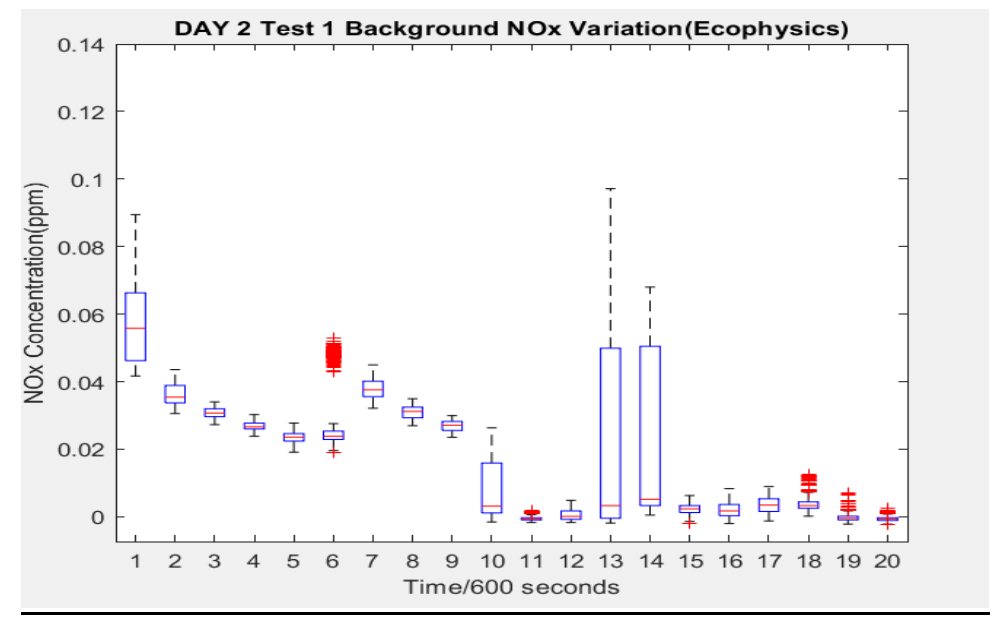

Figure A2 - Day 2 Test 1 Background NOx Variation(EcoPhysics) 


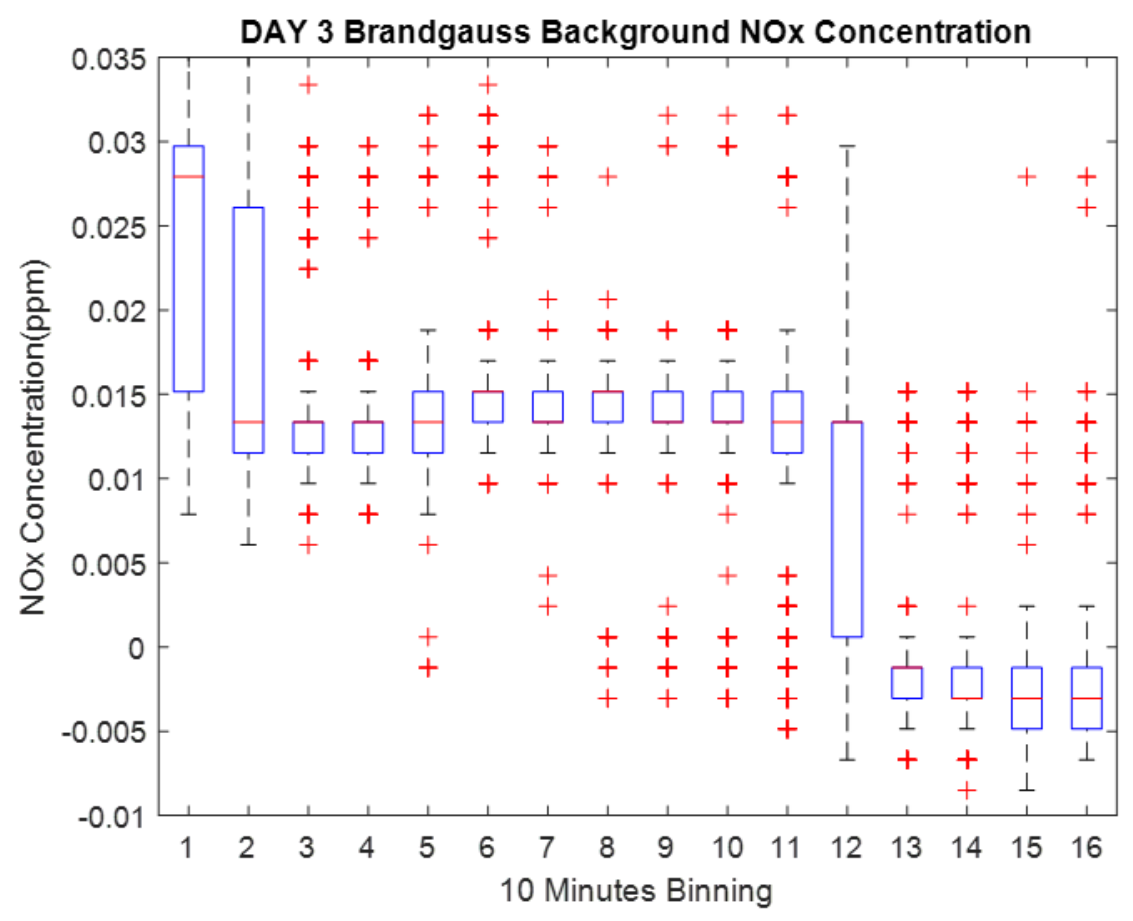

Figure A3 - Day 3 Background NOx Variation(Brandgaus)

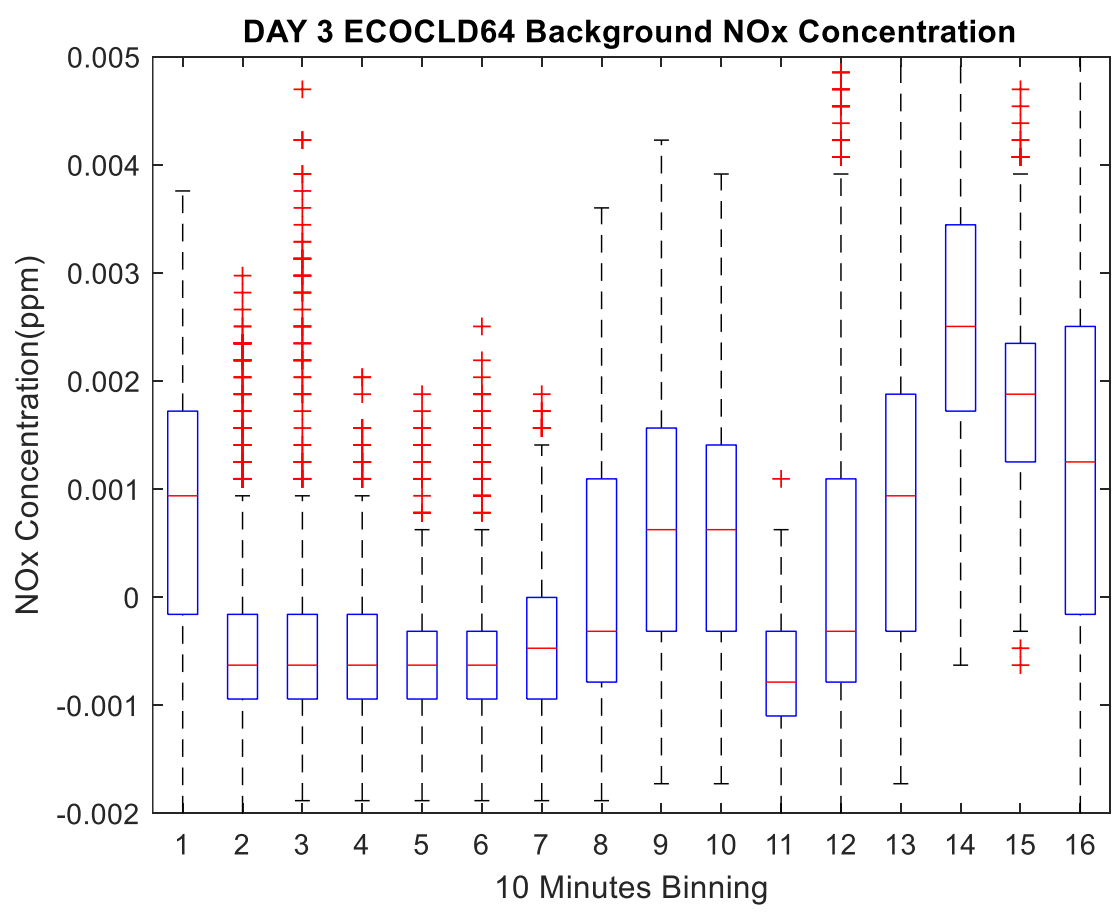

Figure A4 - Day 3 Background NOx Variation(EcoPhysics) 
Low NOx Analyzers Specifications

Table A1 - Brandgaus 7705 NOx Analyzer Specifications

\begin{tabular}{|c|c|}
\hline Parameter & Specifications \\
\hline $\begin{array}{l}\text { Measurement } \\
\text { Technology }\end{array}$ & $\begin{array}{l}\text { Chemiluminescence using all solid-state } \\
\text { detection }\end{array}$ \\
\hline Measurement range & 1 to $500 \mathrm{ppm}$ \\
\hline Zero Noise & $\leq 0.02 \mathrm{ppm}$ \\
\hline Zero Calibration Drift & $\pm 0.1 \mathrm{ppm}$ \\
\hline Span Noise & $\leq 0.25 \%$ of reading \\
\hline Span Calibration drift & $\pm 1 \%$ of reading \\
\hline Linearity Error & $\leq 2 \%$ of full scale \\
\hline Interferences & $<2 \%$ of full scale \\
\hline Response Time & $\mathrm{T}_{95}<15 \mathrm{secs}$ \\
\hline $\begin{array}{l}\mathrm{NO}_{2} \text { Converter } \\
\text { Efficiency }\end{array}$ & $>95 \%$ \\
\hline
\end{tabular}

Table A2 - Ecophysics CLD 64 NOx Analyzer Specifications

\begin{tabular}{|c|c|}
\hline Parameter & Specifications \\
\hline Measuring Ranges & 0.5 to $100 \mathrm{ppm}$ \\
\hline Limit of detection & $2 \mathrm{ppb}$ \\
\hline Noise at Zero point $(1 \sigma)$ & $1 \mathrm{ppb}$ \\
\hline Lag-time & $<1 \mathrm{sec}$ \\
\hline Rise time (0 -90\%) & $<1 \mathrm{sec}$ \\
\hline Temperature Range & $5-40^{\circ} \mathrm{C}$ \\
\hline Humidity Tolerance & $\begin{array}{c}\text { 5-95\% tolerance relative humidity (non- } \\
\text { condensing ambient air and sample gas) }\end{array}$ \\
\hline Sample flow rate & 300 ml/min \\
\hline Input pressure & Ambient \\
\hline Standards & NO/NOx analyzer with internal molybdenum \\
& converter \\
\hline
\end{tabular}




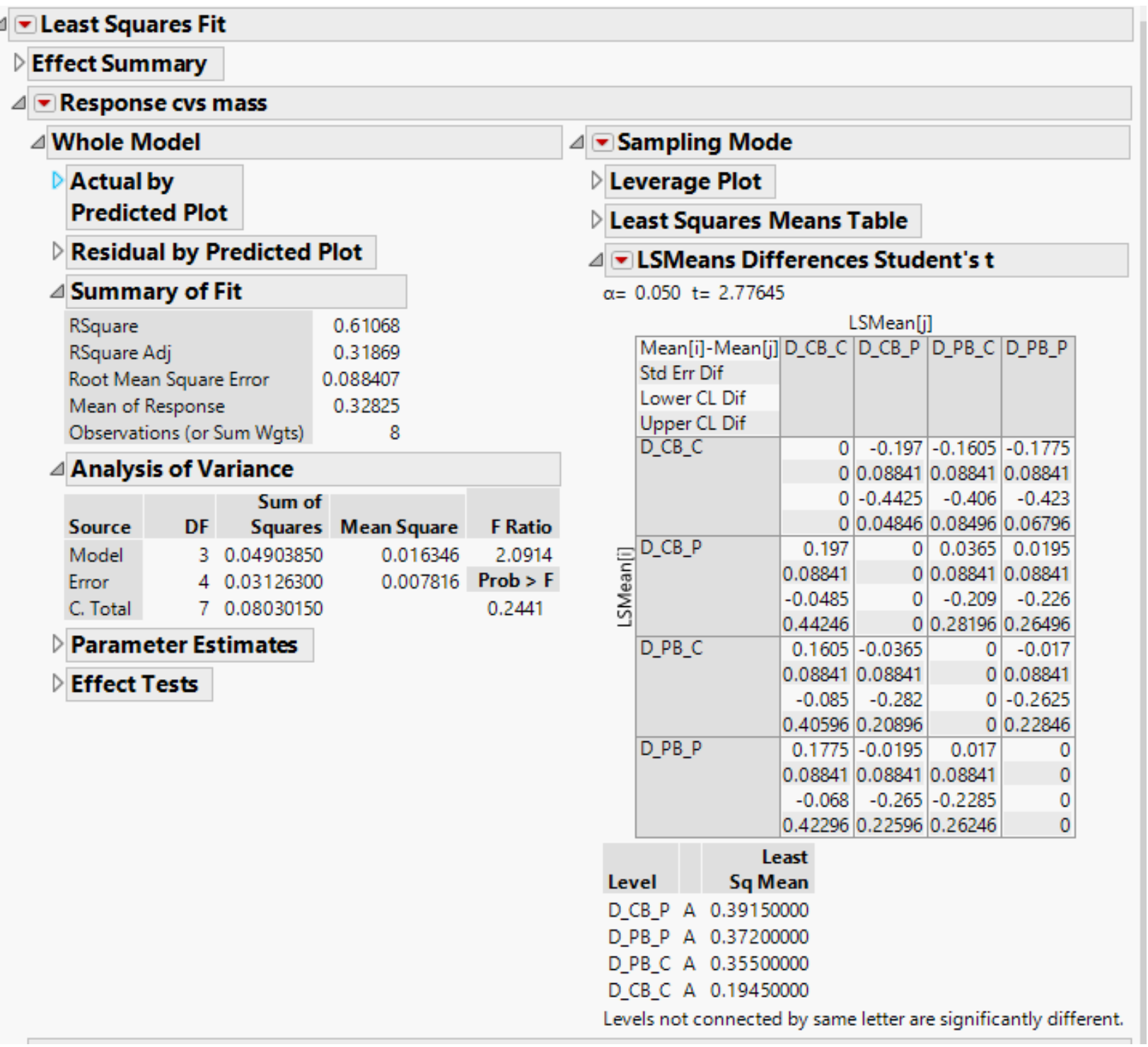

Figure A5 - JMP Output of ANOVA and Student-T test comparison of CVS measurements at four Different Bag Sampling modes. 


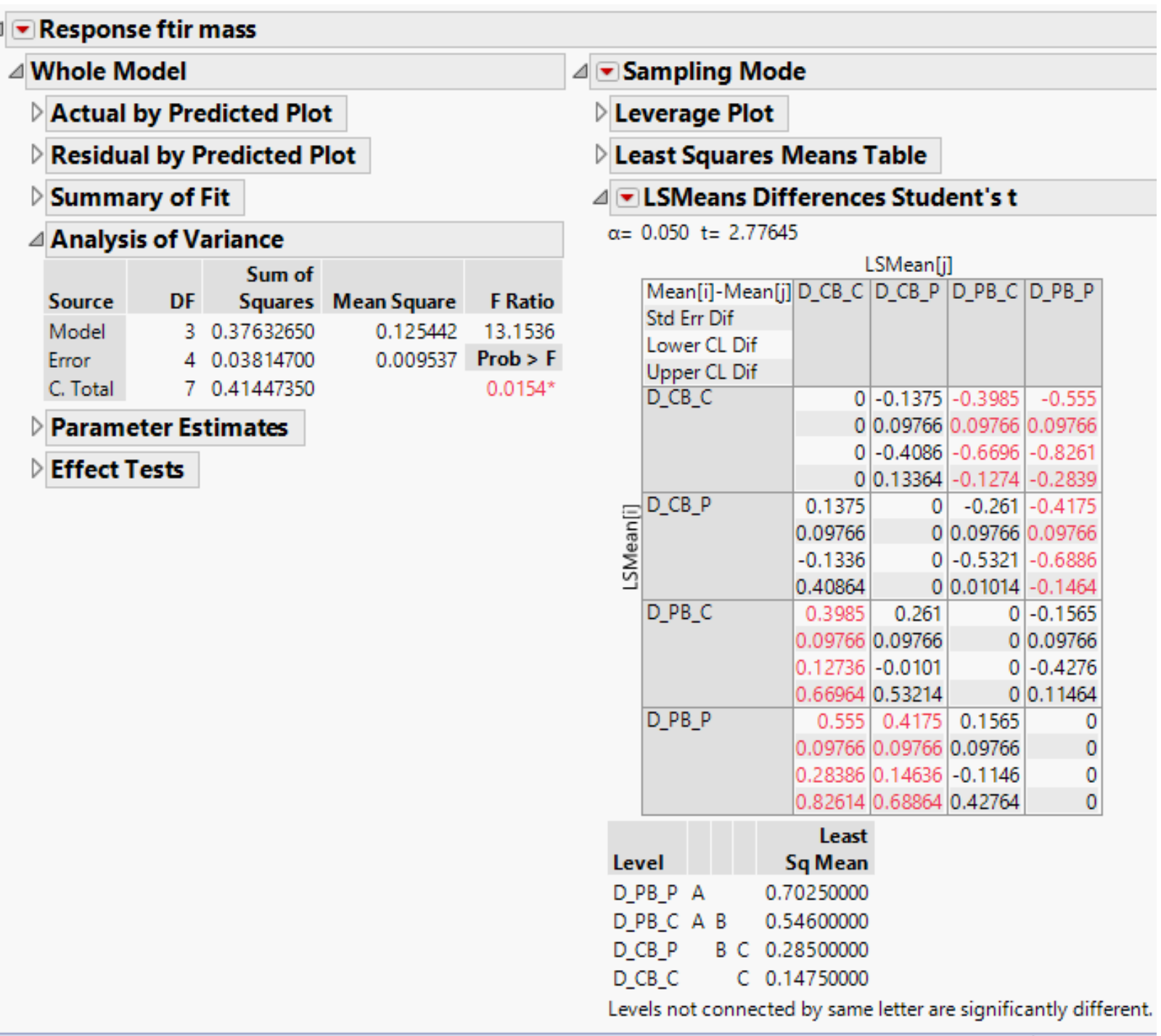

Figure A6- JMP Output ANOVA and Student-T test comparison of FTIR measurements at four Different Bag Sampling modes. 


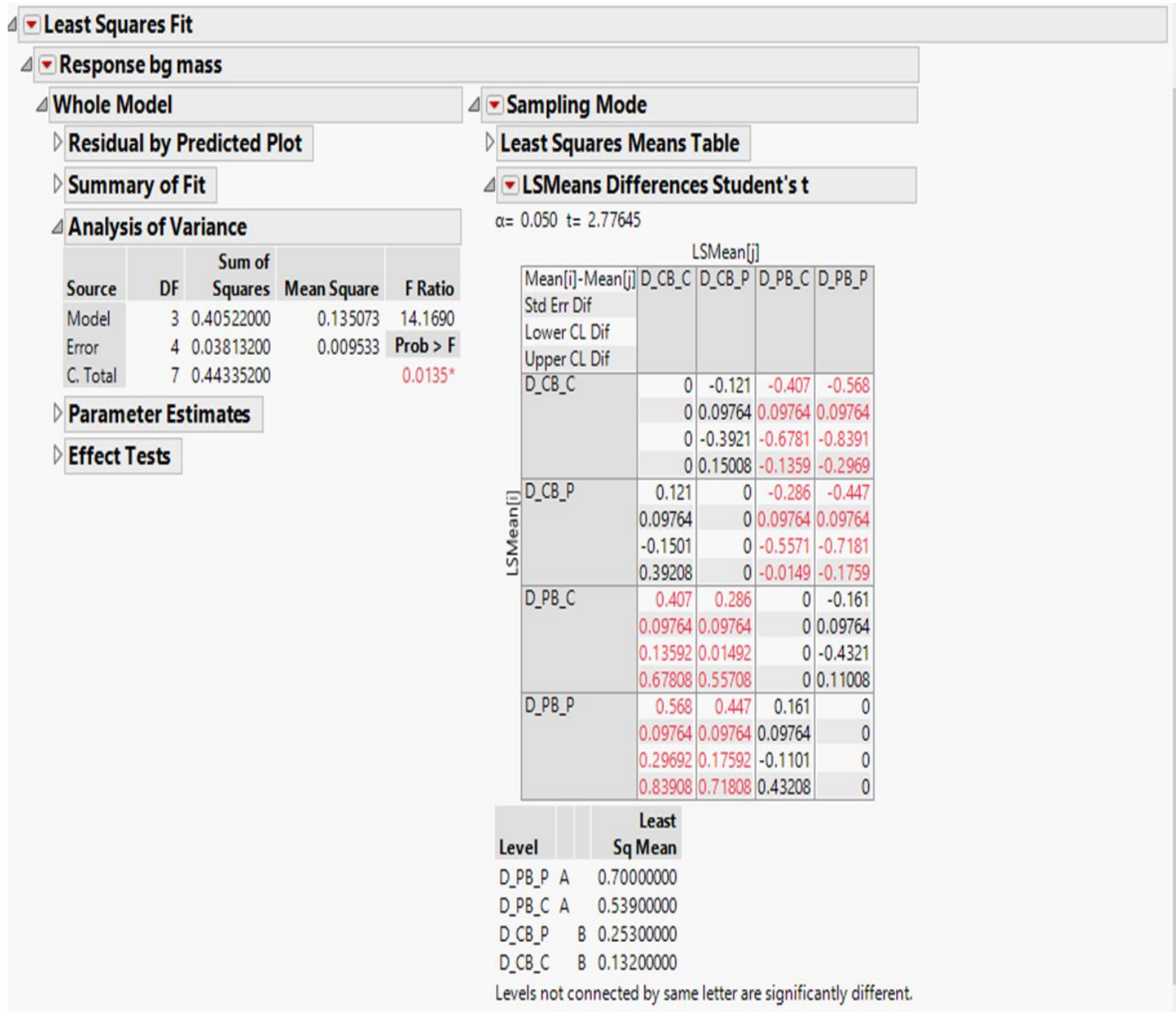

Figure A7 - JMP Output ANOVA and Student-T test comparison of Brandgaus measurements at four Different Bag Sampling modes. 
Levels not connected by same letter are significantly different.

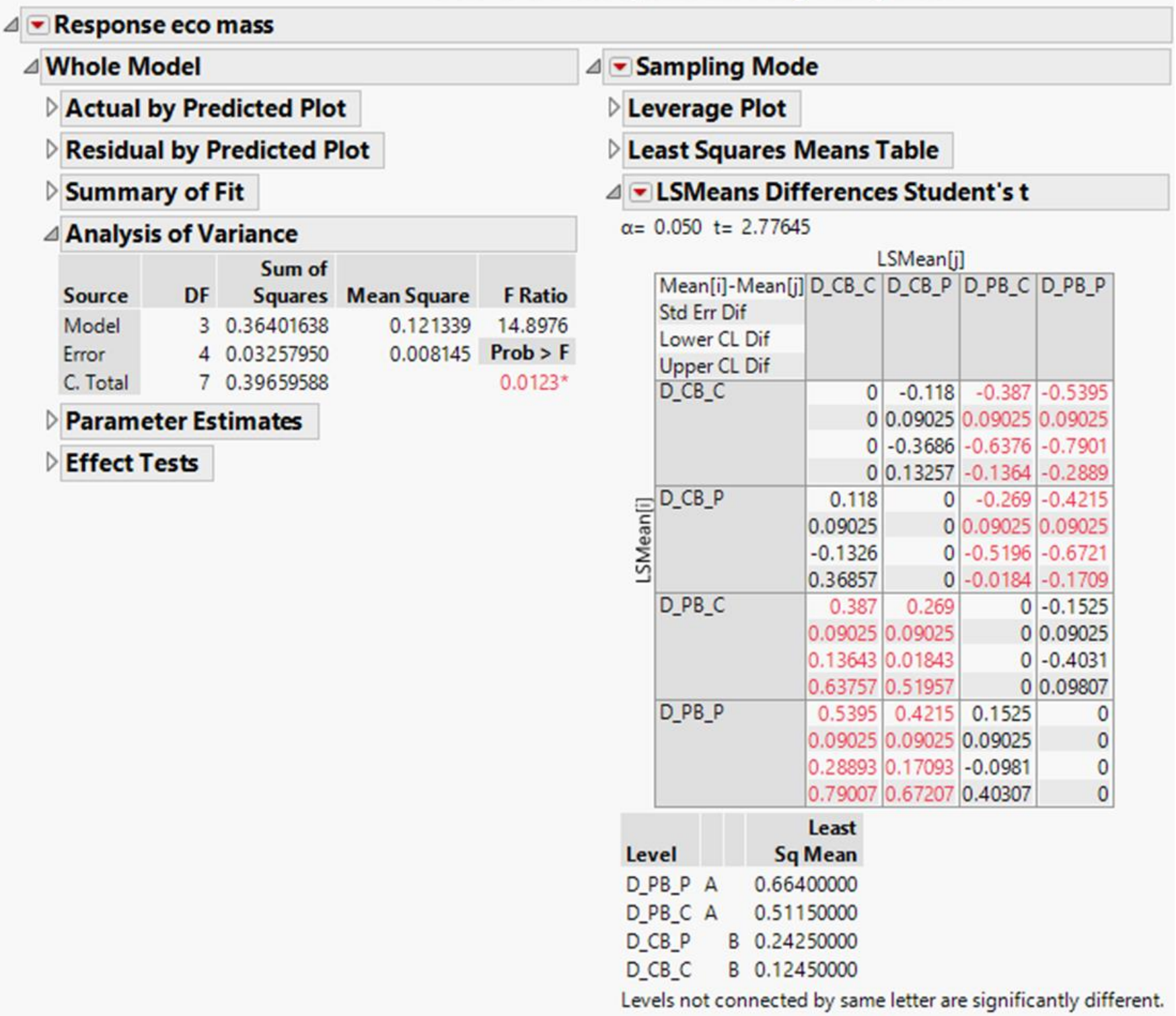

Figure A8- JMP Output ANOVA and Student-T test comparison of Ecophysics measurements at four Different Bag Sampling modes. 


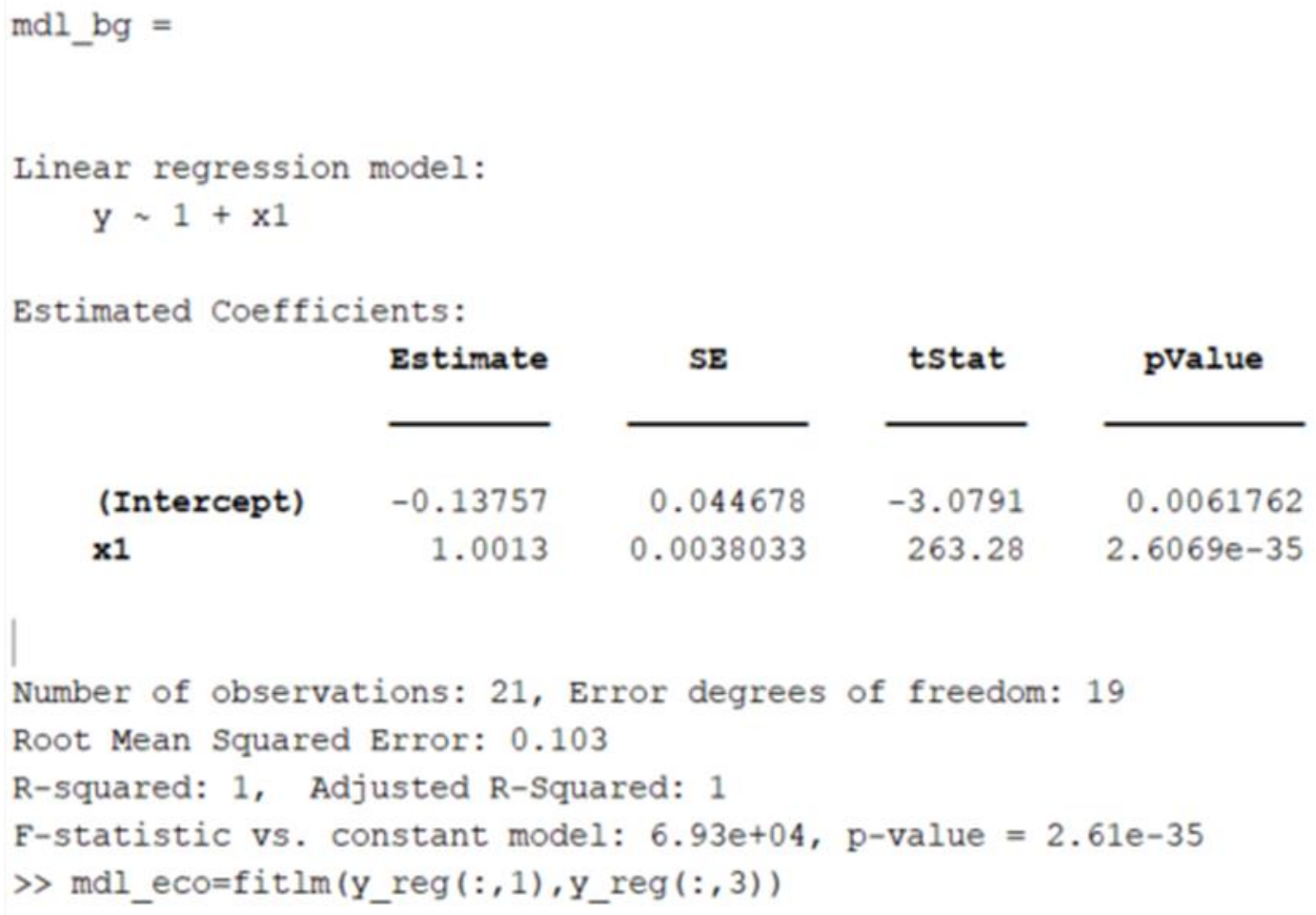

Figure A9 - Summary of Brandgauss Analyzer Linear Regression Model

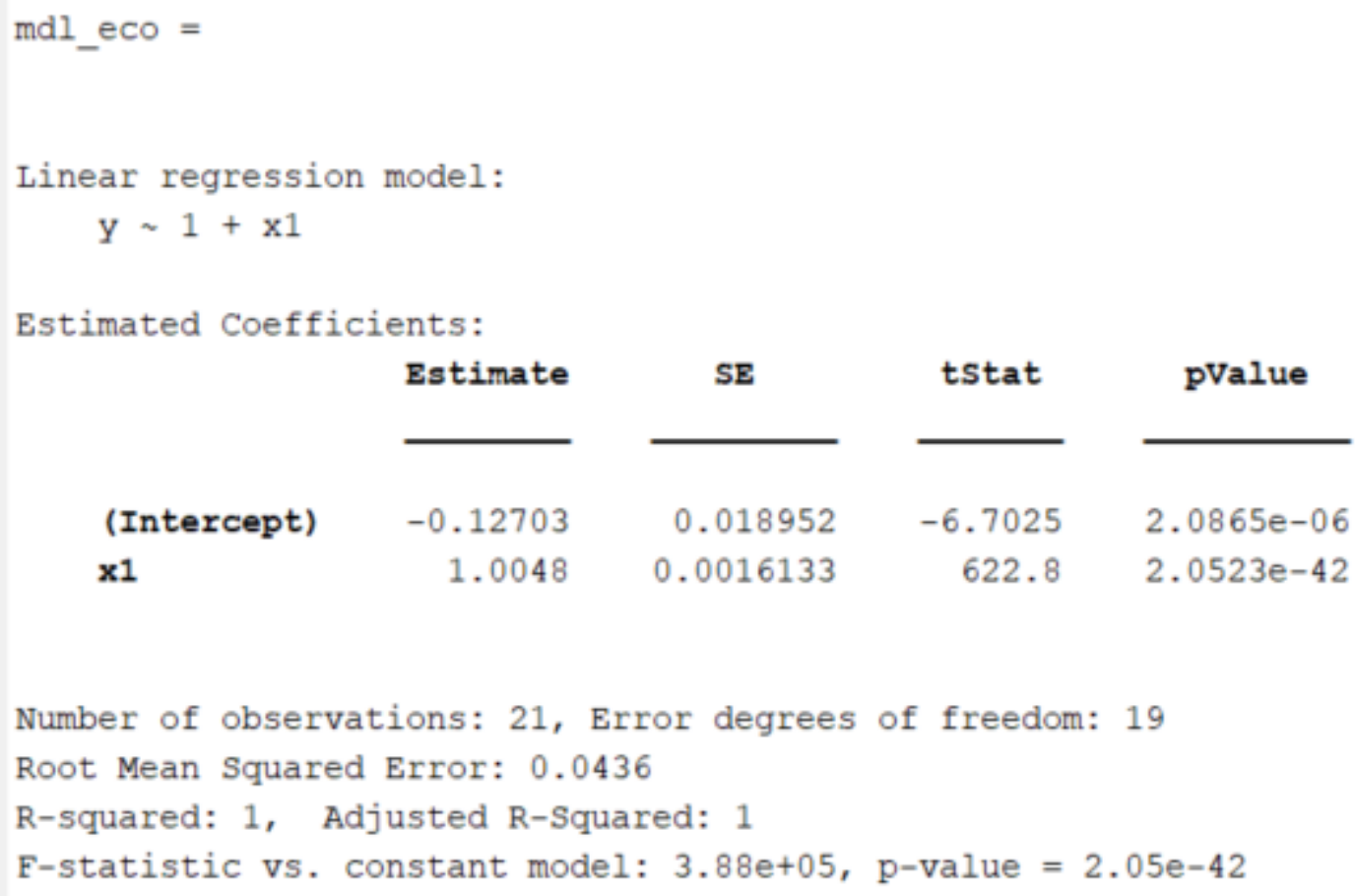

Number of observations: 21, Error degrees of freedom: 19

Root Mean Squared Error: 0.0436

R-squared: 1, Adjusted R-Squared: 1

F-statistic vs. constant model: $3.88 \mathrm{e}+05, \mathrm{p}$-value $=2.05 \mathrm{e}-42$

Figure A10 - Summary of Ecophysics Analyzer Linear Regression model 